\title{
Procédés de chauffage par micro-ondes : approches expérimentales et numériques des interactions avec les produits alimentaires
}

\author{
Microwave heating processes: experimental and numerical approaches \\ to interactions with food products
}

\author{
Sebastien Curet $^{1}$ \\ ${ }^{1}$ GEPEA - ONIRIS - (UMR CNRS 6144), Site de la Géraudière CS 82225, 44322 Nantes cedex 3, France, \\ sebastien.curet@oniris-nantes.fr
}

RÉSUMÉ. Ce document présente les concepts élémentaires des transferts de chaleur relatifs au procédé de traitement thermique par micro-ondes de produits alimentaires. Les paramètres procédés et les propriétés produits sont détaillés en lien avec les différents mécanismes responsables de l'élévation de température sous micro-ondes. Des aspects théoriques relatifs à la propagation d'ondes dans des structures guidées sont aussi présentés en incluant les effets thermiques sur le produit traité.

Un focus est porté sur les différents dispositifs expérimentaux existants pour des applications en recherche et développement ainsi que la modélisation du chauffage par micro-ondes en vue de prédire le comportement thermique du produit au cours du procédé. Enfin, les applications majeures de la technologie de chauffage par micro-ondes sont présentées en incluant à la fois des discussions sur les effets thermiques et non-thermiques des micro-ondes dans les matrices alimentaires.

ABSTRACT. This document presents the basic concepts of heat transfer related to the microwave heating process of food products. The process parameters and product properties are detailed with the different mechanisms responsible for the temperature rise under microwave. Theoretical aspects of wave propagation in guided structures are also presented, including thermal effects on the processed material.

Some focus will be on the various existing experimental devices for research and development applications as well as microwave heating modelling approaches to predict the thermal behaviour of the product during the process. Finally, the major applications of microwave heating technology are presented by including both thermal and non-thermal effects on food materials.

MOTS-CLÉS. micro-ondes, transfert de chaleur, propriétés diélectriques, modélisation, guide d'ondes, effets thermiques et athermiques.

KEYWORDS. microwaves, heat transfer, dielectric properties, modelling, waveguides, thermal and non-thermal effects.

\section{Généralités sur les traitements thermiques par micro-ondes}

L'utilisation du rayonnement micro-ondes pour traiter thermiquement des produits a vu le jour dans les années 1950-1960 avec les premiers développements technologiques issus des travaux de Percy Spencer et Raytheon (Edgar \& Osepchuk, 2001). De nos jours, les applications des micro-ondes sont diverses et variées et concernent des secteurs industriels allant de l'industrie alimentaire à l'automobile par exemple. Au niveau industriel, l'enjeu principal est de réduire le temps de traitement thermique par rapport à un traitement conventionnel, ce qui implique plus de flexibilité au niveau du procédé, ainsi qu'un réduction de la consommation énergétique globale. Les équipements peuvent différer significativement selon la taille de l'installation : simple cavité micro-ondes de quelques centimètres jusqu'à un tunnel de traitement en continu de plusieurs mètres de long.

Selon la nomenclature officielle, le rayonnement micro-ondes est caractérisé par des fréquences variants de $300 \mathrm{MHz}$ à $300 \mathrm{GHz}$ et se situe donc entre le spectre des radiofréquences et les infrarouges (figure 1.1). Les micro-ondes sont à ce titre désignées comme ondes décimétriques à millimétriques (longueurs d'ondes de $1 \mathrm{~m}$ à $1 \mathrm{~mm}$ ). 
Le schéma ci dessous représente le positionnement des bandes de fréquences micro-ondes par rapport à l'ensemble du spectre des ondes électromagnétiques. Il met en relation les longueur d'ondes en fonction de la taille des objets (de l'échelle microscopique à l'échelle humaine).

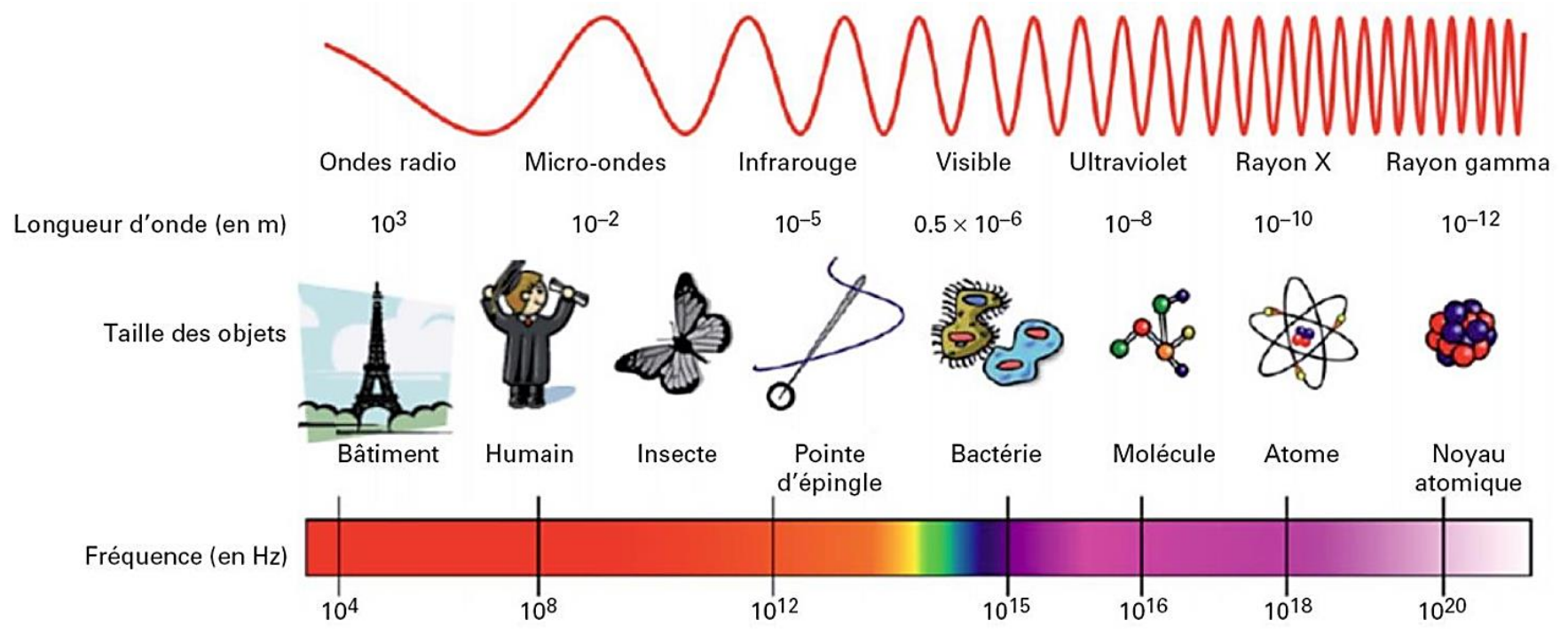

Figure 1.1. Positionnement des micro-ondes dans le spectre fréquentiel (d'après Perino et Chemat, 2015)

Plus particulièrement, le domaine des micro-ondes peut être divisé en trois sous-bandes décrites par décades de fréquence :

- de 0,3 à $3 \mathrm{GHz}$ : bande UHF (Ultra-Hautes Fréquences - ondes décimétriques)

- de 3 à $30 \mathrm{GHz}$ : bande SHF (Supra-Hautes Fréquences - ondes centimétriques)

- de 30 à $300 \mathrm{GHz}$ : bande EHF (Extra-Hautes Fréquences - ondes millimétriques)

Pour les applications dédiées au chauffage par micro-ondes, les fréquences les plus couramment utilisées sont $2,45 \mathrm{GHz}$ (micro-ondes domestiques et applications industrielles, longueur d'onde à vide d'environ $12 \mathrm{~cm}$ ) et $915 \mathrm{MHz}$ (applications industrielles essentiellement, longueurs d'ondes à vide d'environ $33 \mathrm{~cm}$ ).

\section{Propriétés diélectriques}

\subsection{Généralités sur les mécanismes}

Dans le cas du chauffage par micro-ondes de produits alimentaires, l'énergie contenue dans l'onde électromagnétique se dégrade en chaleur au sein de matériaux dits à pertes diélectriques. Pour dégrader cette énergie en chaleur, ces matériaux se doivent d'être constitués de molécules polaires avec des dissymétries de charges.

Les interactions des ondes électromagnétiques avec une matrice sont caractérisées par les propriétés diélectriques intrinsèques du produit traité. Ces propriétés diélectriques sont définies par une permittivité complexe constituée d'une partie réelle, la constante diélectrique ou permittivité relative, et d'une partie imaginaire, le facteur de pertes diélectriques, selon la relation suivante :

$$
\varepsilon=\varepsilon^{\prime}-j \varepsilon^{\prime \prime}=\varepsilon_{0}\left(\varepsilon_{r}^{\prime}-j \varepsilon_{r}^{\prime \prime}\right)
$$

La constante diélectrique ainsi que le facteur de pertes sont souvent normalisés par rapport aux caractéristiques diélectriques du vide, $\varepsilon_{0}$, ce qui permet de définir des propriétés diélectriques relatives (constantes diélectriques et facteur de pertes) adimensionnelles. 
La constante diélectrique traduit la faculté d'une molécule à s'orienter dans le sens du champ électrique (phénomène de polarisation) (İçier and Baysal, 2004a) alors que le facteur de pertes diélectriques caractérise la dégradation en chaleur de l'énergie contenue dans l'onde électromagnétique (Zhu et al., 2012).

Les phénomènes de frictions intermoléculaires dûs à la rotation des dipôles électriques se manifestent par un léger retard pris par les matériaux polarisés pour s'orienter après l'application du champ électrique. Ce déphasage est représenté par l'angle de pertes $\delta$ qui relie le facteur de pertes à la permittivité réelle. Le rapport du facteur de pertes diélectrique relatif à la constante diélectrique relative définie la tangente à l'angle de perte (Chandrasekaran et al., 2013) et est exprimé par :

$$
\tan \delta=\frac{\varepsilon_{r}^{\prime \prime}}{\varepsilon_{r}^{\prime}}
$$

Les mécanismes de génération de chaleur sous micro-ondes sont différents suivant le type de fréquence considérée dans la bande UHF. Ces mécanismes sont illustrés sur la figure 2.1 ci-après.

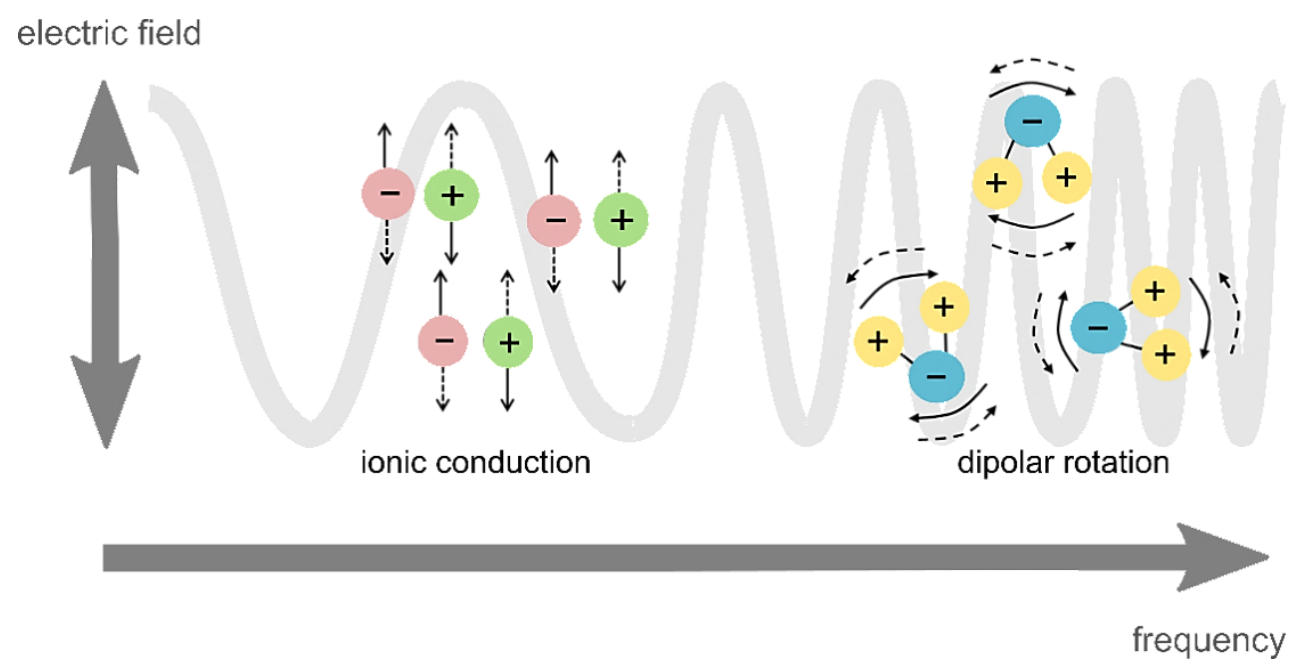

Figure 2.1. Représentation schématique des deux principaux mécanismes liés au chauffage par micro-ondes dans la bande UHF : la rotation dipolaire et la conduction ionique

Le premier mécanisme est la rotation dipolaire. Ce mécanisme dépend de l'existence de molécules polaires dans le matériau. Dans le cas des produits alimentaires, l'eau est généralement la composante polaire majeure. Pour la molécule d'eau, les différences d'électronégativités des atomes individuels conduisent à l'existence d'un dipôle électrique permanent (Meda et al., 2005). Lorsqu'un champ électromagnétique oscillant est généré, les molécules d'eau tendent à se réaligner suivant la direction du champ électrique. En raison du champ électrique à haute fréquence, ce réalignement se produit un million de fois par seconde et provoque un frottement interne des molécules conduisant à un échauffement volumique du produit (Chandrasekaran et al., 2013).

Le deuxième mécanisme est la polarisation ionique ou conduction ionique. La chaleur est générée par la migration oscillatoire des ions sous l'action d'un champ électrique à haute fréquence (Datta et Davidson, 2000). Ces mouvements ioniques entrainent des collisions entre les ions et d'autres molécules, entraînant la conversion de l'énergie cinétique des ions en énergie thermique (SalazarGonzález et al., 2012).

En fonction de la fréquence micro-ondes utilisée, les mécanismes responsables de l'élévation de température diffèrent. Ainsi dans la bande de fréquences micro-ondes UHF, en particulier autour de $300 \mathrm{MHz}$, les phénomènes de conduction ionique dans les matrices alimentaires sont majoritairement responsables de l'élévation de température par rapport à la rotation dipolaire. Dans ce cas, la 
dégradation de l'énergie de l'onde électromagnétique en chaleur est due à la conductivité électrique des ions présents dans les matrices. A l'inverse, à la fréquence de $2450 \mathrm{MHz}$, les mécanismes lies à la rotation dipolaire des molécules sont majoritaires (Kubo et al, 2018a).

Pour illustrer la prédominance des ces deux mécanismes, la figure 2.2 propose une représentation suivant la fréquence micro-ondes considérée (Tang, 2015).

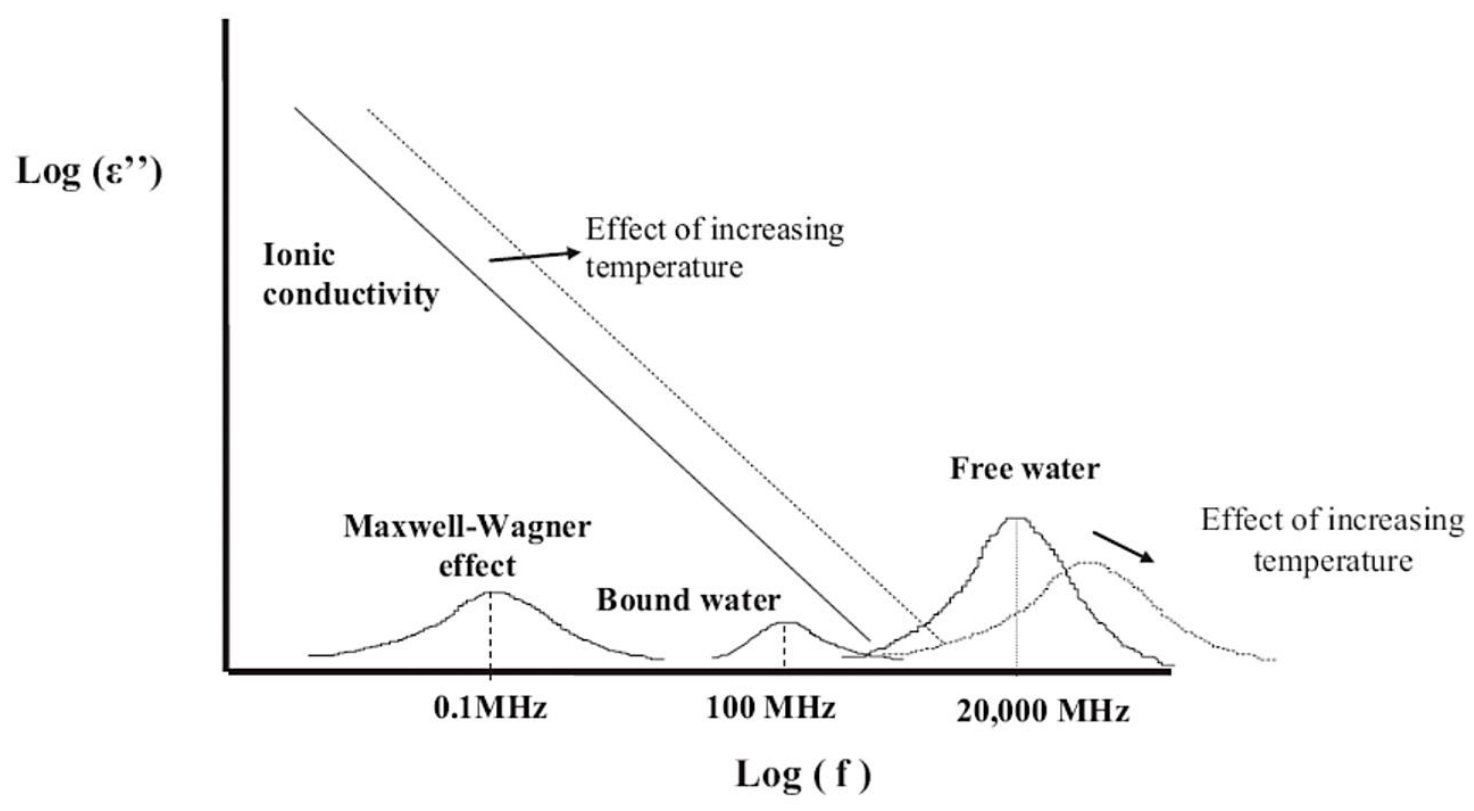

Figure 2.2. Contribution des différents mécanismes responsables de la génération de chaleur par microondes dans des produits alimentaires en fonction de la fréquence (d'après Tang, 2015)

Dans le domaine des radiofréquences (bandes « $\mathrm{HF}$ » high frequency, $3 \mathrm{MHz}-30 \mathrm{MHz}$ et « VHF » Very High Frequency, $30 \mathrm{MHz}-300 \mathrm{MHz}$ ), on remarque que le facteur prépondérant responsable de l'élévation de température sous micro-ondes est la conductivité ionique du produit. A titre d'exemple, la conductivité ionique d'une matrice alimentaire augmente avec la teneur en sel dissous dans le milieu, ce qui induit en général une augmentation de la chaleur générée.

Si l'on passe dans la bande UHF, la contribution de la conductivité ionique au mécanisme de chauffage par micro-ondes est peu importante au dessus de la fréquence de $300 \mathrm{MHz}$. Dans ce cas, le mécanisme de rotation dipolaire devient prépondérant. 




Figure 2.3. Contribution de la conductivité électrique et de la relaxation de l'eau libre par rapport au facteur de perte global d'une solution de chlorure de sodium aqueuse 0,5N (adapté de Roebuck et Goldblith, 1972)

Comme l'illustre la figure 2.3 dans le cas d'une solution saline $0.5 \mathrm{~N}$, les mécanismes de rotation dipolaire et de conduction ionique sont les mécanismes de pertes diélectriques dominants à 2450 et $915 \mathrm{MHz}$. Dans ce cas, aux fréquences micro-ondes d'intérêt pour les applications de chauffage (400 $3000 \mathrm{MHz}$ ), le facteur de pertes diélectriques dévie de la décroissance linéaire classique $\varepsilon_{r}{ }^{\prime}=f$ (fréquence) habituellement observée en échelle log-log (figure 2.2).

$\mathrm{Au}$ final, compte tenu de la variabilité de ces mécanismes, on définit souvent dans le cas d'un produit alimentaire, un facteur de pertes diélectriques effectif qui regroupe les différents mécanismes en fonction de la fréquence des micro-ondes considérée.

$$
\varepsilon^{\prime \prime}{ }_{r \text { eff }}=\varepsilon^{\prime \prime}{ }_{r(p)}+\varepsilon^{\prime \prime}{ }_{r(\sigma)}
$$

Le terme $\varepsilon^{\prime \prime}{ }_{r(p)}$ traduit le facteur de pertes relatif aux mécanismes de polarisation des molécules d'eau (rotation dipolaire). Le terme $\varepsilon^{\prime \prime}{ }_{r(\sigma)}$ traduit le facteur de pertes relatif au mécanisme de conduction ionique. Il est dépendant de la conductivité électrique $\sigma$ mesurable dans le système. La conductivité électrique $(\sigma)$ est en lien avec le facteur de pertes diélectriques relatif et caractérise les propriétés conductrices des électrons libres.

$$
\sigma=\omega \varepsilon_{0} \varepsilon^{\prime \prime}{ }_{r(\sigma)}
$$

Avec:

$$
\varepsilon^{\prime \prime}{ }_{r e f f}=\varepsilon^{\prime \prime}{ }_{r(p)}+\frac{\sigma}{\omega \varepsilon_{0}}
$$

L'ensemble des pertes par polarisation est généralement regroupé sous un terme unique car les méthodes de caractérisation diélectriques existantes distinguent difficilement les différentes contributions (figure 2.2).

$$
\varepsilon^{\prime \prime}{ }_{r(p)}=\varepsilon^{\prime \prime}{ }_{r \text { (Mawwell-Wagner })}+\varepsilon^{\prime \prime}{ }_{r \text { (dipolaire) }}+\varepsilon^{\prime \prime}{ }_{r \text { (atomique })}+\varepsilon^{\prime \prime}{ }_{r \text { (electronique) }}
$$


En général, il existe trois façons qualitatives de classer les matériaux en fonction de leur absorption d'énergie sous micro-ondes: i) les absorbeurs ou matériaux à pertes diélectriques élevées qui s'échauffent facilement sous micro-ondes ; ii) les matériaux transparents ou à faibles pertes diélectriques dans lesquels les micro-ondes traversent le matériau avec peu ou pas d'atténuation ; iii) les matériaux opaques ou conducteurs qui réfléchissent les micro-ondes à leur surface. Par conséquent, la connaissance des propriétés diélectriques est nécessaire pour différencier les matériaux dans les catégories ci-dessus (Chandrasekaran et al., 2013 ; Yin, 2012).

\subsection{Notion de profondeur de pénétration}

La relation entre les propriétés diélectriques et l'intensité de la puissance micro-ondes à l'intérieur du produit est caractérisée par la profondeur de pénétration. La profondeur de pénétration par rapport à la puissance micro-ondes est définie comme la distance dans le produit à laquelle la puissance incidente est réduite à $1 / \mathrm{e}(\mathrm{e}=2,7183)$ de sa valeur à la surface, c'est-à-dire qu'environ $63 \%$ de la puissance incidente est réduite entre la surface et cette distance (figure 2.4).

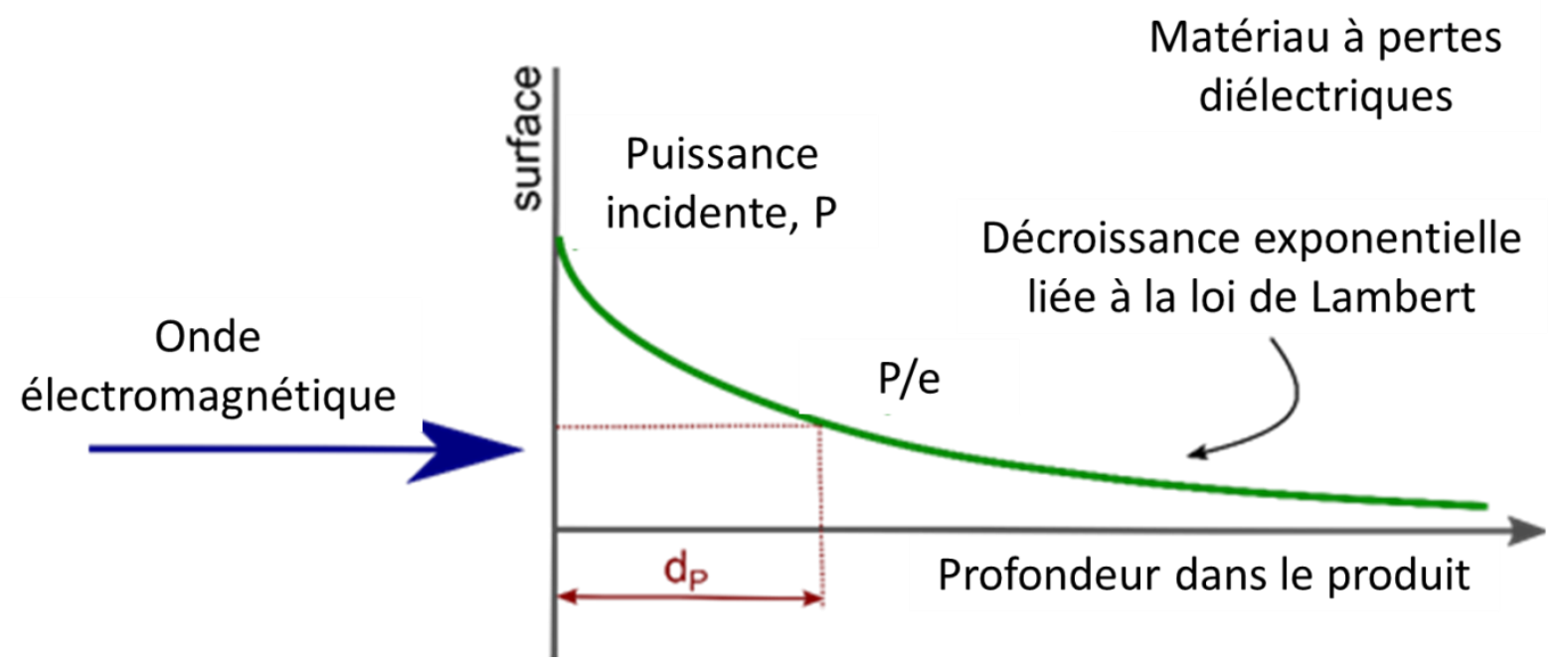

Figure 2.4. Représentation schématique d'une profondeur de pénétration de la puissance micro-ondes au sein d'un matériau à pertes diélectriques. Adapté de Komarov et al (2005)

Dans un produit alimentaire, la profondeur de pénétration de la puissance micro-ondes peut être calculée connaissant les valeur des propriété diélectriques du produit selon l'équation suivante (Curet et al., 2008a :; Sosa-Morales et al., 2010) :

$$
d_{p}=\frac{c_{0}}{2 . \pi \cdot f}\left[2 . \varepsilon_{r} \cdot \cdot\left(\left(1+\left(\frac{\varepsilon_{r}^{\prime \prime}}{\varepsilon_{r}^{\prime}}\right)^{2}\right)^{1 / 2}-1\right)\right]^{-1 / 2}
$$

où $d_{P}$ est la profondeur de pénétration, $\varepsilon_{r}^{\prime}$ et $\varepsilon_{r}^{\prime \prime}$ sont les valeurs de la constante diélectrique relative et du facteur de pertes du produits, $c_{0}$ est la vitesse de la lumière en espace libre $\left(2,9979 \times 10^{8} \mathrm{~m} \mathrm{~s}^{-1}\right)$ et $f$ est la fréquence de l'onde électromagnétique considérée.

La valeur de la profondeur de pénétration des micro-ondes en lien avec l'épaisseur du produit peut être un paramètre utile lors de la conception d'un produit alimentaire pour optimiser son chauffage par micro-ondes et aussi lors de l'évaluation de l'uniformité de chauffage (Tang, 2015). Par exemple, la figure 2.5 illustre les plages représentatives des profondeurs de pénétration pour certains groupes d'aliments selon leur état physique. 


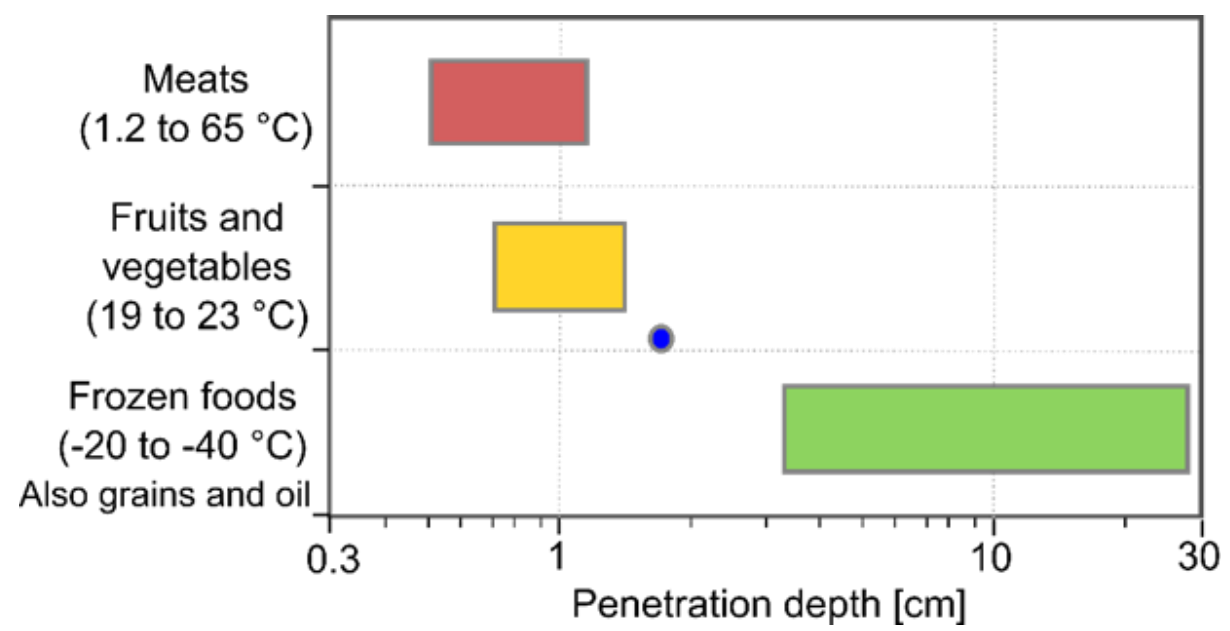

Figure 2.5. Gamme représentative des valeurs de profondeur de pénétration de la puissance micro-ondes selon le type de produit alimentaire (adapté de Datta, 2001)

La figure précédente indique en particulier que la profondeur de pénétration augmente de manière importante pour un produit à l'état congelé par rapport à un produit frais.
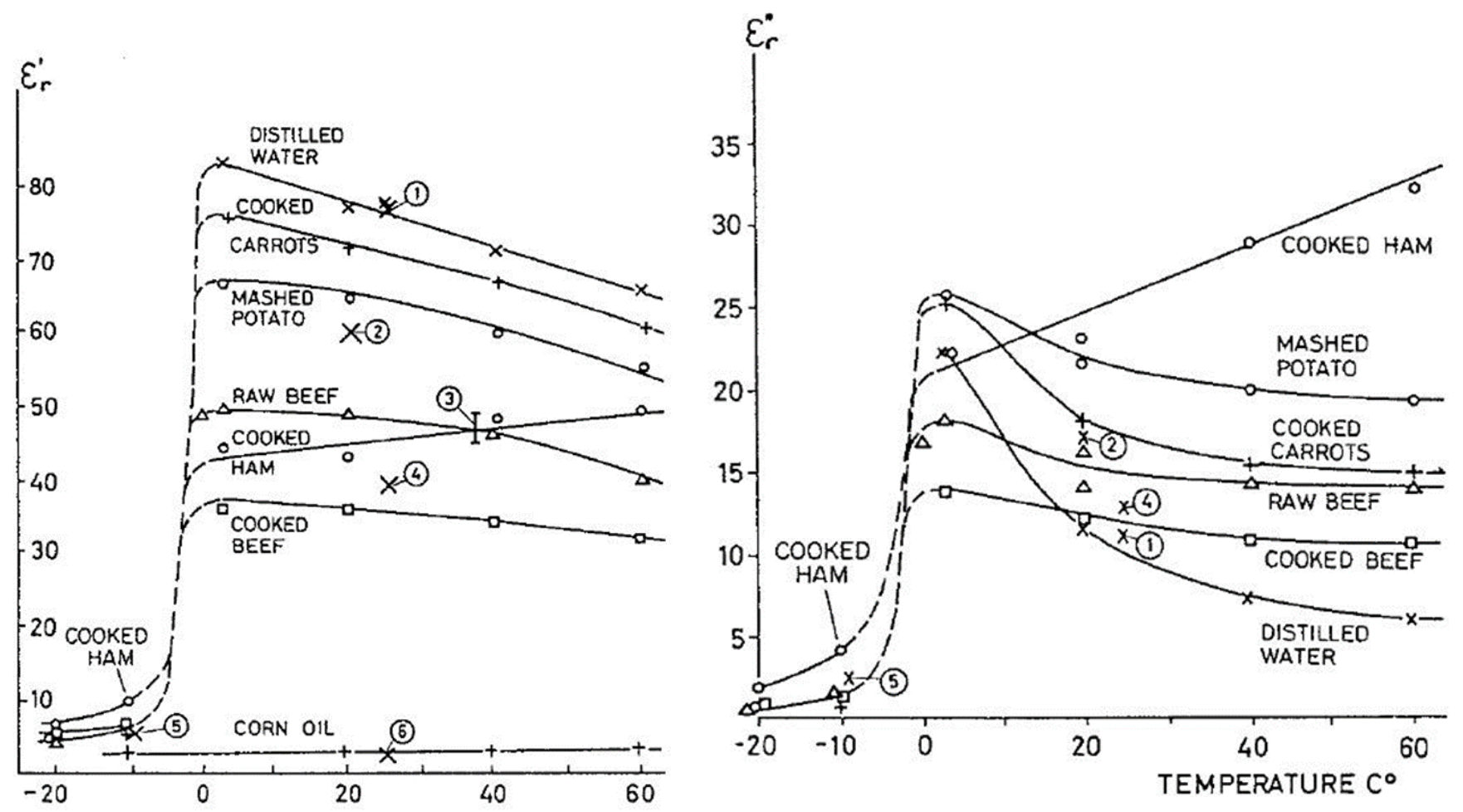

Figure 2.6. Dépendance des propriétés diélectriques en fonction du type de produit et de la température (d'après Bengtsson et Risman, 1971)

L'illustration ci-dessus (figure 2.6) présente l'influence du type de produit et de la température sur les propriétés diélectriques (constante diélectrique et facteur de pertes) dans le cas des produits alimentaires, à la fréquence de 2,45 GHz.

D’une manière générale, la constante diélectrique augmente avec la teneur en eau dans le produit (de l'huile de maïs jusqu'à l'eau distillée). En complément, lors d'un changement de phase (décongélation), une forte augmentation des propriétés diélectriques (constante diélectrique et facteur de pertes) est observée de part et d'autre de la température de fusion (passage état congelé $=>$ décongelé). C'est ce qui explique les différentes importantes de profondeur de pénétration des ondes entre un produit congelé et décongelé. 
Dans le cas du jambon cuit (cooked ham) à l'état décongelé, les propriétés diélectriques ont tendance à augmenter avec la température du fait de la teneur en sel dans le produit.
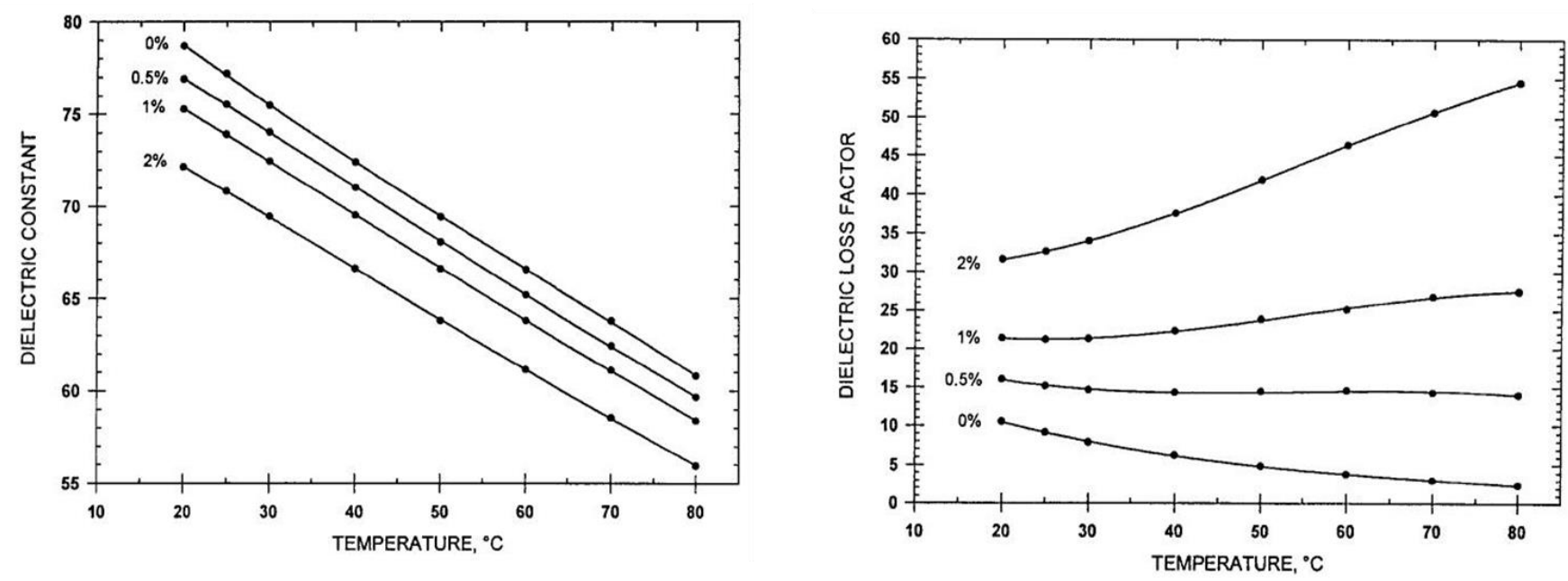

Figure 2.7. Dépendance des propriétés diélectriques en fonction de la composition du produit et de la température (d'après Stogryn, 1971)

L'illustration de la figure 2.7 présente l'influence de la teneur en sel et de la température sur les propriétés diélectriques (constante diélectrique et facteur de pertes) dans le cas d'une solution saline $(\mathrm{NaCl}+$ eau $)$ à la fréquence de $2,45 \mathrm{GHz}$.

D'une manière générale, le fait d'augmenter la concentration en sel au sein d'un liquide ou un produit solide, induit des charges électriques conductrices additionnelles qui en conséquence augmentent les pertes diélectriques du système du fait d'une migration des charges.

\subsection{Mesure des propriétés diélectriques}

Afin de choisir la technique appropriée pour la mesure des propriétés diélectriques, certains facteurs doivent être pris en compte. Parmi ceux-ci, les caractéristiques des matériaux diélectriques à mesurer, la gamme de fréquences, le degré de précision requis, la disponibilité des équipements et des ressources pour les investigations à mener (İçier et Baysal, 2004b ; Nelson et Datta, 2001). La figure 2.8 présente certaines techniques et leurs gammes de fréquences respectives de mesure. En général, trois grandes familles sont généralement désignés pour caractériser les propriétés diélectriques des produits, à savoir : la ligne de transmission, la cavité résonante et la sonde coaxiale ouverte.

Pour mesurer les propriétés diélectriques, plusieurs méthodes de référence existent en fonction de différents critères (type de produit, état physique, fréquence du spectre micro-ondes...). 


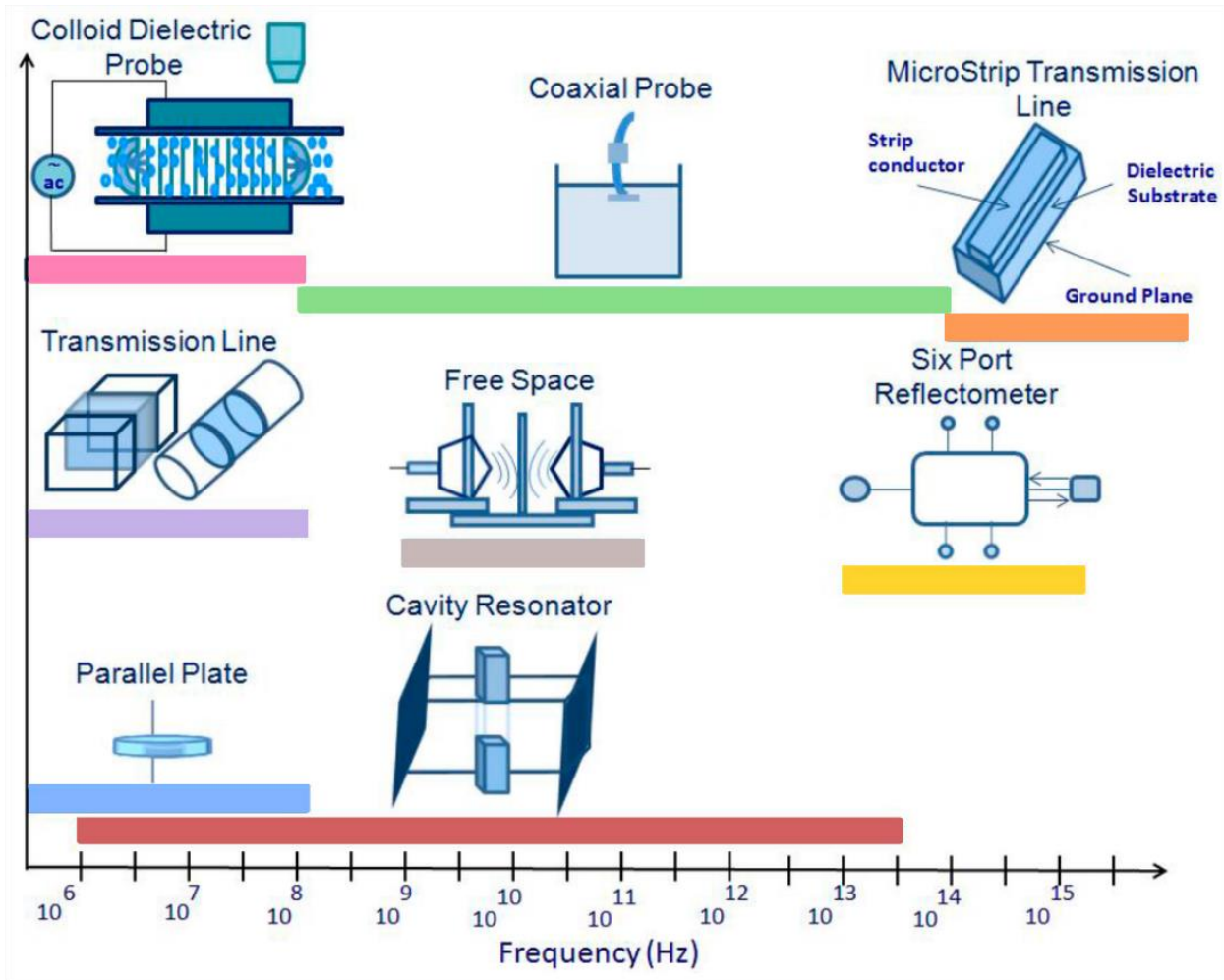

Figure 2.8. Techniques habituelles de mesure des propriétés diélectriques, en fonction de la gamme de fréquences (adapté de Khaled et al, 2016)

Dans la technique de la ligne de transmission, les propriétés diélectriques sont mesurées par transmission ou réflexion d'ondes au travers une ligne dont la section transversale est entièrement remplie par l'échantillon à caractériser (İçier et Baysal, 2004b ; Sosa-Morales et al., 2010). Habituellement, trois principaux types de lignes de transmission sont utilisés comme cellule de mesure : le guide d'ondes rectangulaire, la ligne coaxiale et la ligne à microruban (Komarov et al., 2005). Cette méthode convient particulièrement bien aux échantillons solides ou semi-solides. Dans le cas d'échantillons liquides et de solides divisés, le système doit être configuré verticalement, en tenant compte de la formation de ménisque à la surface des liquides et du compactage des particules. Les propriétés diélectriques des gaz sont trop faibles pour être caractérisées par cette méthode (İçier et Baysal, 2004b). La précision de la méthode de la ligne de transmission se situe généralement entre celle de la méthode de la sonde coaxiale ouverte et celle de la méthode de la cavité résonnante (Komarov et al., 2005). En raison des exigences concernant la forme et la taille des échantillons, leur préparation est relativement difficile (İçier et Baysal, 2004b ; Komarov et al., 2005).

La méthode de la cavité résonante est basée sur une analyse comparative de certaines caractéristiques électromagnétiques entre une cavité résonnante cylindrique ou rectangulaire à vide et partiellement chargée par le produit à caractériser (Komarov et al., 2005). Dans cette méthode, la préparation des échantillons est relativement facile et un grand nombre d'échantillons peut être caractérisé en peu de temps. La méthode de la cavité résonante est également facilement adaptable à des températures élevées ou basses et est précise et sensible (İçier et Baysal, 2004b). Cependant, elle ne fournit pas autant de données pour de larges bandes de fréquences. De plus, l'analyse et l'exploitation des données peut se révéler complexe car une modélisation électromagnétique du dispositif expérimental est souvent nécessaire (İçier et Baysal, 2004b). Lorsque les mesures sont requises à une seule fréquence, la technique de la cavité résonante peut cependant devenir un choix raisonnable (Nelson et Datta, 2001).

Actuellement, la méthode de la sonde coaxiale ouverte est l'une des techniques les plus répandue pour mesurer les propriétés diélectriques. Elle est pratique et facile à utiliser, rapide, idéale pour les liquides et les produits semi-solides, non destructive pour de nombreux matériaux, à large bande 
fréquencielle (gammes des radiofréquences à micro-ondes), et il n'y a pas de préparation complexe des échantillons à prévoir (İçier et Baysal, 2004b; Komarov et al., 2005). Cette méthode a été utilisée avec succès pour mesurer des matériaux liquides et semi-solides présentant des pertes diélectriques relativement élevées, ce qui est le cas de la plupart des produits alimentaires (Nelson et Datta, 2001). La méthode de la sonde coaxiale ouverte n'est pas recommandée pour les matériaux à très faibles pertes diélectriques et elle est sujette à des erreurs en cas de variations importantes de densité à l'intérieur du matériau. Cette technique exige une épaisseur d'échantillon supérieure à $1 \mathrm{~cm}$ et une surface plane dans les échantillons solides pour éviter les erreurs dues à la présence de bulles d'air entre l'extrémité de la sonde coaxiale et la surface de l'échantillon (İçier et Baysal, 2004b ; Nelson et Datta, 2001). En général, la surface des échantillons solides doit présenter une rugosité inférieure à $0,5 \mu \mathrm{m}$ pour minimiser les erreurs de mesure (Arai et al., 1995). La précision de cette technique est généralement limitée $( \pm 5 \%)$, mais des résultats suffisamment précis peuvent être obtenus pour les matériaux à pertes diélectriques moyennes et élevées (İçier et Baysal, 2004b; Komarov et al, 2005). Le système de mesure de cette méthode est basé sur une ligne coaxiale cylindrique ouverte qui est excitée par une onde électromagnétique transversale (TEM). Les paramètres (amplitude et phase) des signaux incidents et réfléchis sont détectés par un analyseur de réseau connecté à la sonde (Komarov et al., 2005). La permittivité complexe est déterminée en fonction du facteur de réflexion des ondes, qui est généralement calculé via un algorithme intégré au niveau logiciel. Afin d'éliminer l'influence du signal réfléchi causé par les discontinuités dans la ligne de transmission, une procédure d'étalonnage est nécessaire. La calibration consiste généralement à mesurer les propriétés de l'air (diélectrique parfait), d'une ligne de transmission court-circuitée ( $100 \%$ de puissance réfléchie) et d'un matériau diélectrique comme l'eau distillée (ou tout autre matériau dont les propriétés diélectriques sont bien connues).

Pour résumer, les deux techniques les plus répandues restent la technique de la sonde coaxiale ouverte (Chen, Pitchai et al. 2014) et les cavités résonnantes (Kraszewski and Nelson 1996). La première technique est surtout utilisée dans le cas de produits à fortes pertes diélectriques (typiquement les produits dont le facteur de pertes est supérieur à 1). À l'inverse la technique de la cavité résonnante reste utilisée pour les produits faiblement absorbants sous micro-ondes (matières grasses, produits pulvérulents, ...). Dans ce cas, cette technique est adaptée pour des produits dont le facteur de pertes peut être inférieur à 0,5 ou encore plus faible. Comme mentionné précédemment, chaque technique dispose de ses propres avantages et inconvénients. La sonde coaxiale ouverte couvre cependant un large spectre d'étendues de mesures diélectriques pour les produits alimentaires. Cette technique reste largement développée commercialement avec de nombreux fabricants proposant des solutions tout en un.

\section{Propagations d'ondes en espace libre et au sein de milieux diélectriques}

\subsection{Equations de propagation}

Les équations de Maxwell décrivent la relation entre un champ électrique et magnétique variant dans le temps et l'espace. Associées à des conditions aux limites appropriées, ces équations décrivent mathématiquement le comportement des champs électromagnétiques à l'intérieur d'une cavité microondes (Dibben, 2001 ; Komarov et al., 2005). Les équations de Maxwell sous forme différentielle sont la représentation la plus utilisée pour résoudre les études d'électromagnétisme et sont présentées comme suit (Curet et al., 2008b) :

$$
\begin{aligned}
& \nabla \times \boldsymbol{H}=\frac{\partial \boldsymbol{D}}{\partial t}+\boldsymbol{J}=\frac{\partial \varepsilon \boldsymbol{E}}{\partial t}+\sigma \boldsymbol{E} \\
& \nabla \times \boldsymbol{E}=-\frac{\partial \boldsymbol{B}}{\partial t}=-\frac{\partial \mu \boldsymbol{H}}{\partial t}
\end{aligned}
$$




$$
\begin{aligned}
& \nabla \cdot \boldsymbol{D}=\nabla \cdot \boldsymbol{\varepsilon} \boldsymbol{E}=\rho_{\boldsymbol{e}} \\
& \nabla . \boldsymbol{B}=\nabla \cdot \mu \boldsymbol{H}=0
\end{aligned}
$$

$\varepsilon$ et $\mu$ représentent respectivement la permittivité et la perméabilité des milieux. Ces valeurs sont généralement normalisées par rapport à la permittivité et la perméabilité du vide égales respectivement à $\varepsilon_{0}=(36 \pi)^{-1} \times 10^{-9} \mathrm{~F} / \mathrm{m}$ et $\mu_{0}=4 \pi \times 10^{-7} \mathrm{H} / \mathrm{m}$.

La première équation est la loi de Maxwell Ampère et la deuxième représente la loi de Maxwell Faraday. La troixième équation décrit la loi de Gauss et la dernière équation traite le champ d'induction magnétique $\boldsymbol{B}$. Chacune de ces quatre équations décrit un phénomène physique.

La loi de Maxwell Ampère exprime la manière dont un courant électrique $\boldsymbol{J}$ est à l'origine d'un champ magnétique $\boldsymbol{H}$. Cette équation indique physiquement qu'un courant ou l'induction électrique $\boldsymbol{D}$ variant dans le temps génère un champ magnétique $\boldsymbol{H}$ en rotation. D'une manière similaire, l'équation de Maxwell Faraday montre que l'induction magnétique $\boldsymbol{B}$ qui varie avec le temps génère un champ électrique $\boldsymbol{E}$ en rotation (champ électromoteur). Cette équation illustre particulièrement bien le principe du phénomène d'induction.

Dans les équations présentées, l'opérateur mathématique de la divergence $(\nabla)$ est une mesure du flux total qui émerge d'un point donné. Ainsi, l'équation basée sur la loi de Gauss, exprime la manière dont les charges électriques sont à l'origine du champ électrique. Plus précisément, elle indique que la divergence de l'induction électrique en un point est proportionnelle à la densité de charge électrique $\rho_{e}$.

Par analogie avec la loi de Maxwell Gauss, la dernière équation pour le flux magnétique $\boldsymbol{B}$ montre qu'il n'y a pas de source ou puit de champ magnétique, ou plus simplement qu'il n'existe pas de charges magnétiques.

L'ensemble des quatre équations de Maxwell s'applique dans un vide en présence de charges et de courants. Ces équations sont pour autant distinctes des équations de Maxwell dans la matière. En effet, dans les milieux réels, certaines charges sont libres de se déplacer tandis que d'autres sont liées entre elles pour former atomes et molécules; les équations de Maxwell sont alors différentes. Dans les milieux diélectriques sans charges libres ni courant libres (diélectriques parfaits linéaires isotropes et homogènes), les équations de Maxwell deviennent :

$$
\begin{aligned}
& \nabla \times \boldsymbol{H}=\frac{\partial \boldsymbol{D}}{\partial t}=\frac{\partial \varepsilon \boldsymbol{E}}{\partial t} \\
& \nabla \times \boldsymbol{E}=-\frac{\partial \boldsymbol{B}}{\partial t}=-\frac{\partial \mu \boldsymbol{H}}{\partial t} \\
& \nabla . \boldsymbol{D}=\nabla . \varepsilon \boldsymbol{E}=0 \\
& \nabla . \boldsymbol{B}=\nabla . \mu \boldsymbol{H}=0
\end{aligned}
$$

Pour les milieux diélectriques linéaires homogènes, les inductions $\boldsymbol{D}$ et $\boldsymbol{B}$ sont proportionnelles aux champs électriques $\boldsymbol{E}$ et magnétiques $\boldsymbol{H}$ avec un coefficient de proportionnalité indépendant de l'espace. Dans ces milieux, la forme des équations de Maxwell est la même que dans le vide, seules les valeurs des permittivités et perméabilités changent. 

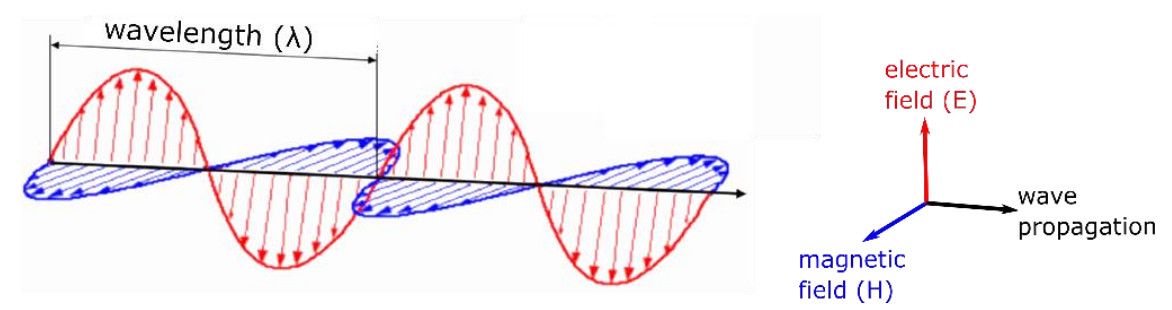

Figure 3.1. Composantes d'une onde électromagnétique. L'onde plane incidente est composée d'un champ électrique et d'un champ magnétique tous deux orthogonaux (adapté de Tang et al, 2015)

Selon la théorie de Maxwell, un champ électrique variable crée un champ magnétique variable qui, à son tour, créer un champ électrique et ainsi de suite. Cette interaction mutuelle est à la base de la génération des ondes électromagnétiques. Il apparaît que le champ magnétique et le champ électrique sont orthogonaux en tous points, et perpendiculaires à la direction de propagation (figure 3.1). De plus, leur intensité oscille simultanément à la fréquence imposée par la source.

La constante de propagation complexe du champ (ou nombre d'ondes) est défini comme suit (Pozar, 2011) :

$$
\gamma=j \omega \sqrt{\mu \varepsilon} \sqrt{1-j \frac{\sigma}{\omega \varepsilon}} \text { avec } \varepsilon=\varepsilon^{\prime}-j \varepsilon^{\prime \prime}=\varepsilon_{0}\left(\varepsilon_{r}^{\prime}-j \varepsilon_{r}^{\prime \prime}\right)
$$

La constante de propagation peut être présentée de manière différente, telle que :

$$
\begin{aligned}
& \gamma=\left[(j \omega \sqrt{\mu \varepsilon})^{2}\left(1-j \frac{\sigma}{\omega \varepsilon}\right)\right]^{0.5}=\left[-\omega^{2} \mu \varepsilon\left(1-j \frac{\sigma}{\omega \varepsilon}\right)\right]^{0.5} \\
& \gamma=\left[-\omega^{2} \mu \varepsilon+\omega^{2} \mu \varepsilon\left(j \frac{\sigma}{\omega \varepsilon}\right)\right]^{0.5}=\left[-\omega^{2} \mu \varepsilon+\omega \mu(j \sigma)\right]^{0.5} \\
& \text { or } \gamma^{2}=-\omega^{2} \mu \varepsilon\left(1-j \frac{\sigma}{\omega \varepsilon}\right) \\
& \text { ainsi } \gamma^{2}=\left[-\omega^{2} \mu \varepsilon+\omega \mu(j \sigma)\right]=-\omega^{2} \mu \varepsilon+j \sigma \omega \mu
\end{aligned}
$$

Au sein de milieux diélectriques à pertes sans charges libres et courants conducteurs, l'équation de propagation du champ électrique se réduit à (Pozar, 2011 ;Cherbanski and Rudniak 2013) :

$$
\begin{aligned}
& \nabla^{2} E-\gamma^{2} E=0 \\
& \nabla^{2} E+\omega^{2} \mu \varepsilon\left(1-j \frac{\sigma}{\omega \varepsilon}\right) E=0 \text { avec } \varepsilon=\varepsilon_{0} \varepsilon_{r}^{\prime}-j \varepsilon_{0} \varepsilon^{\prime \prime}{ }_{r} \text { et } \mu=\mu_{0} \mu_{r}
\end{aligned}
$$

Cette équation peut être explicitée de la manière suivante : 


$$
\begin{aligned}
& \nabla^{2} E+\omega^{2} \mu_{r} \mu_{0} \varepsilon_{0}\left(\varepsilon_{r}^{\prime}-j \varepsilon^{\prime \prime}{ }_{r}\right)\left(1-j \frac{\sigma}{\omega \varepsilon_{0}\left(\varepsilon_{r}^{\prime}-j \varepsilon^{\prime \prime}{ }_{r}\right)}\right) E=0 \\
& \Leftrightarrow \nabla^{2} E+\omega^{2} \mu_{r} \mu_{0} \varepsilon_{0}\left(\left(\varepsilon^{\prime}{ }_{r}-j \varepsilon^{\prime \prime}{ }_{r}\right)-j \frac{\left(\varepsilon^{\prime}{ }_{r}-j \varepsilon^{\prime \prime}{ }_{r}\right) \sigma}{\omega \varepsilon_{0}\left(\varepsilon_{r}^{\prime}-j \varepsilon^{\prime \prime}{ }_{r}\right)}\right) E=0 \\
& \Leftrightarrow \nabla^{2} E+\omega^{2} \mu_{r} \mu_{0} \varepsilon_{0}\left(\left(\varepsilon_{r}^{\prime}-j \varepsilon^{\prime \prime}{ }_{r}\right)-j \frac{\sigma}{\omega \varepsilon_{0}}\right) E=0 \\
& \Leftrightarrow \nabla^{2} E+\mu_{r} k_{0}^{2}\left(\varepsilon_{r}^{\prime}-j\left(\varepsilon^{\prime \prime}{ }_{r}+\frac{\sigma}{\omega \varepsilon_{0}}\right)\right) E=0 \text { avec } k_{0}=\omega \sqrt{\varepsilon_{0} \mu_{0}}
\end{aligned}
$$

$k_{0}$ est la constante de propagation en espace libre $\left(\mathrm{m}^{-1}\right)$. Si l'on fait intervenir la conductivité électrique effective incluant les deux principaux mécanismes responsables de l'échauffement sous micro-ondes, cela conduit à :

$$
\sigma_{\text {eff }}=\omega \cdot \varepsilon_{0} \varepsilon_{\text {reff }}^{\prime \prime}=\omega \cdot\left(\varepsilon_{\text {polarization }}^{\prime \prime}+\varepsilon_{\text {conduction }}^{\prime \prime}\right)=\omega \cdot\left(\varepsilon_{\text {polarization }}^{\prime \prime}+\frac{\sigma}{\omega}\right)=\omega \cdot \varepsilon_{0}\left(\varepsilon_{r(p)}^{\prime \prime}+\frac{\sigma}{\omega \varepsilon_{0}}\right)
$$

L'équation de propagation du champ électrique devient donc :

$$
\nabla^{2} E+\mu_{r} k_{0}^{2}\left(\varepsilon_{r}^{\prime}-j \varepsilon^{\prime \prime}{ }_{r e f f}\right) E=0 \quad \text { avec } k_{0}=\omega \sqrt{\varepsilon_{0} \mu_{0}}
$$

Par analogie avec la permittivité qui définit le lien avec le champ électrique, la perméabilité décrit les interactions avec le champ magnétique. Dans la plupart des applications micro-ondes, les matériaux traités sont non magnétiques et la perméabilité peut être considérée comme égale à celle du vide. Ceci est particulièrement vérifié dans le cas des produits alimentaires qui contiennent de très faibles quantités de matériaux magnétiques tels que le fer ou le cobalt. La perméabilité magnétique d'un produit alimentaire peut donc être considérée comme égale à celle du vide $\left(\mu_{r}=1\right.$ avec $\left.\mu=\mu_{0}=4 \pi \times 10^{-7} \mathrm{H} / \mathrm{m}\right)$.

\subsection{Notion de propagation guidée}

Une onde électromagnétique, en espace libre se déplace sans limitation dans toutes les directions mais il est possible de la conditionner pour contrôler sa direction de propagation et augmenter son rendement énergétique. Comme présenté sur la figure 3.2, il existe plusieurs supports pour guider une onde (lignes bifilaires, câble coaxial, circuit imprimé, guide d'ondes creux; fibre optique). 

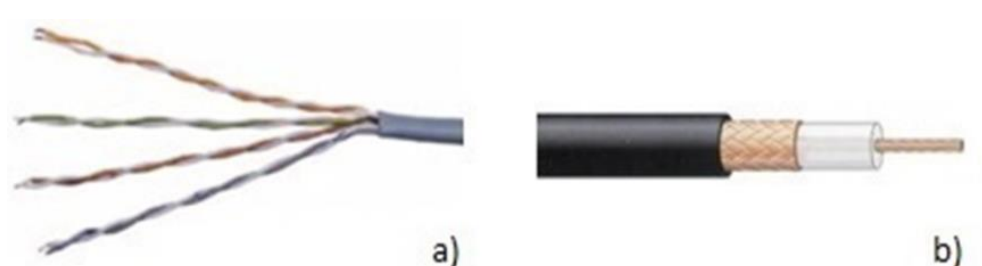

a)

b)
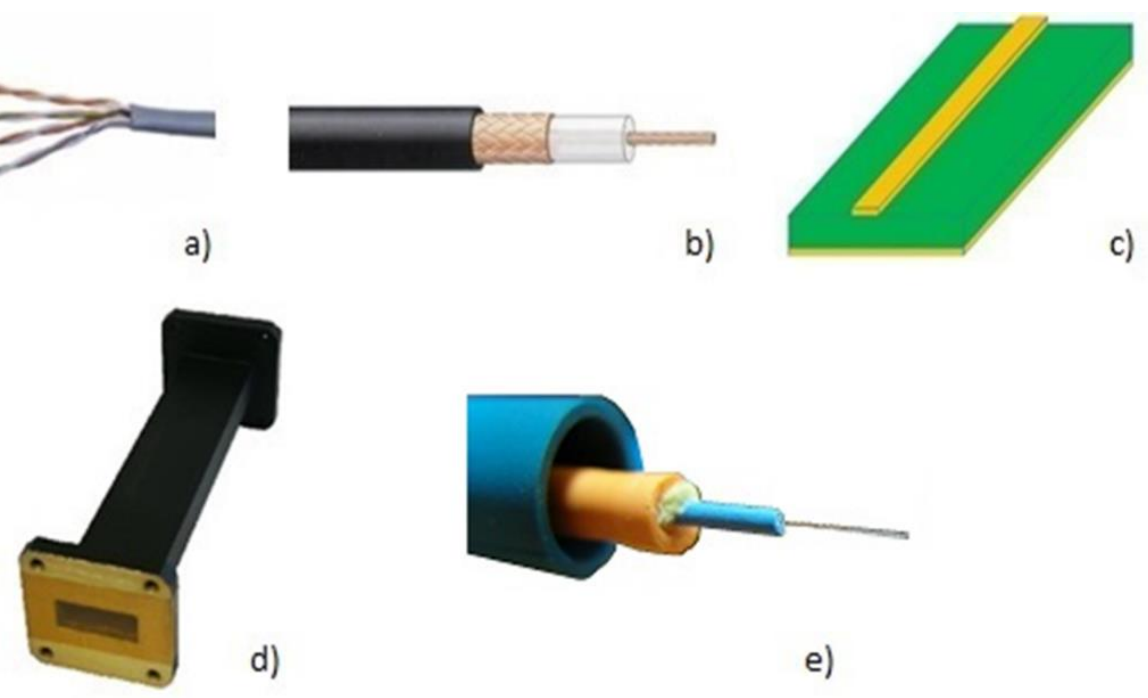

e)

Figure 3.2. Différents types de guides d'ondes (d'après Sadot, 2018)

Dans le cas d'applications industrielles du chauffage micro-ondes, le guide d'ondes creux de section rectangulaire et le câble coaxial sont les plus souvent utilisés car ils sont les seuls dispositifs pouvant transmettre une grande quantité d'énergie. Les autres exemples de guides d'ondes cités servent à transmettre un signal et non à transporter de l'énergie à forte puissance.

Aux fréquences micro-ondes, le guide d'ondes rectangulaire présente de faibles pertes de puissance et, par conséquent, il est surtout adapté dans les applications nécessitant la transmission d'énergie importante (Regier et Schubert, 2005). Le guide d'ondes est une structure métallique qui est utilisée pour propager les ondes électromagnétiques dans un espace confiné afin de guider la propagation. Le guide d'ondes est en métal de sorte que les parois réfléchissent presque idéalement les ondes électromagnétiques à l'intérieur du guide (conducteur électrique parfait).

Il existe différents modes de propagation des ondes dans les cavités micro-ondes, à la fois en monomode ou en multimode.

Un mode de propagation dit multimode se caractérise par une distribution complexe des champs électrique et magnétique au sein d'une cavité.

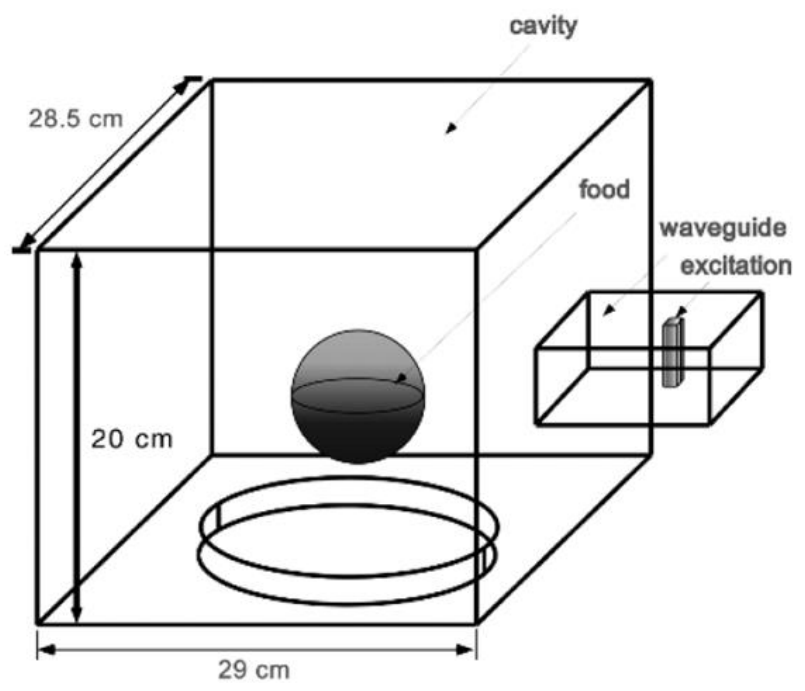

Figure 3.3. Représentation schématique d'une cavité micro-ondes multimode (d'après Datta, 2001) 
Dans l'exemple mentionné sur la figure 3.3, la propagation de l'onde s'effectue au sein d'une cavité micro-ondes (exemple du four à micro-ondes domestique). On distingue la présence d'un guide d'ondes rectangulaire sur la partie droite de l'équipement (cavité monomode pour l'onde incidente), l'onde électromagnétique est ensuite transférée dans la cavité multimode de plus grande taille. Dans ce cas, la répartition des champs électromagnétiques est dépendante des multiples réflexions d'ondes au niveau des parois de la cavité, ainsi que de la charge à traiter dans le produit (propriétés diélectriques, forme géométrique, etc.). Pour une taille de cavité fixée, il est difficile de prédire de manière analytique la forme du champ électromagnétique (nécessité d'avoir recours à la simulation numérique).

Un mode de propagation dit monomode se caractérise quant à lui par une distribution bien définie des champs électromagnétiques dans la cavité. Les modes les plus courants sont plus communément désignés par «transverse électrique » (TE) et «transverse magnétique » (TM). Par définition, les axes $x$ et $y$ sont respectivement parallèles aux grand et petit côtés du guide, la propagation est établie le long de l'axe $z$. La convention habituelle consiste à associer l'axe $x$ au grand côté du guide d'ondes $(a)$ tel que $\mathrm{a}>\mathrm{b}$ (figure 3.4).

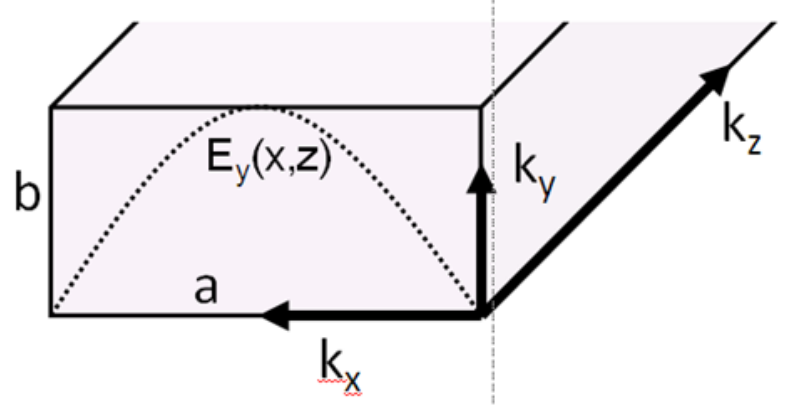

Figure 3.4. Représentation géométrique des composantes du champ électrique dans le cas d'un mode de propagation en TE10 dans un guide d'ondes rectangulaire

Les ondes planes en mode Transverse Electric (TE), ou «H-waves », sont caractérisées par une composante nulle du champ électrique dans le sens de la propagation $\mathrm{z}\left(E_{z}=0\right)$. A l'opposé la composante du champ magnétique est quant à elle non nulle dans cette même direction $\left(H_{z} \neq 0\right)$, d'où la dénomination d'ondes transverse au plan de propagation de l'onde.

A l'opposé, en mode TM, le champ magnétique n'a pas de composante dans la direction de propagation $\left(H_{z}=0\right)$, mais le champ électrique est non nul dans cette direction $\left(E_{z} \neq 0\right)$.

Le cas où ni le champ électrique ni le champ magnétique ne sont transverses correspond au mode hybride TEM. Dans ce mode, les champs électrique et magnétique n'ont aucune composantes dans la direction de propagation des ondes $\left(H_{z}=E_{z}=0\right)$. Le mode TEM ne peut pas exister au sein d'un guide d'ondes rectangulaire.

Afin d'assurer un plus grand transfert d'énergie, les dimensions du guide d'ondes sont choisies de telle sorte que la propagation de l'onde se fasse selon un mode unique, par exemple le mode fondamental TE01 ou TE10. Les nombres d'indices indiquent le nombre de variations semisinusoïdales dans les directions $x$ et $y$, respectivement. Ainsi, le mode TE10 a une variation semisinusoïdale dans la direction $x$ et est constant dans la direction $y$. Le mode TE10 est l'un des modes de propagation les plus courants dans les guides d'ondes rectangulaires, dont la largeur $a$ est égale au double de la hauteur $b$.

Deux paramètres principaux vont définir les conditions de propagation d'une onde électromagnétique au sein d'un guide ; la longueur d'onde de coupure $\lambda_{c}$ et la fréquence de coupure $f_{c}$. Ces deux entités sont liées entre elles par la célérité $C$ de l'onde dans le milieu : 


$$
f_{c}=\frac{C}{\lambda_{c}}
$$

avec $C=\frac{1}{\sqrt{\varepsilon \mu}}$

Pour le mode de propagation TE $\left(E_{x}=E_{z}=0\right)$ au sein du guide d'ondes, l'équation générale de propagation du champ électrique peut être simplifiée telle que :

$$
\left(\frac{\partial^{2} E_{y}}{\partial x^{2}}+\frac{\partial^{2} E_{y}}{\partial z^{2}}\right)+\mu_{r} k_{0}^{2}\left(\varepsilon_{r}^{\prime}-j \varepsilon^{\prime \prime}{ }_{r e f f}\right) E_{y}=0 \text { avec } k_{0}=\omega \sqrt{\varepsilon_{0} \mu_{0}}
$$

Dans le cas d'une propagation guidée, la constante de propagation complexe de l'onde est définie par (Pozar, 2011) :

$$
\gamma^{2}=k^{2}-k_{c}^{2}
$$

$k$ est le nombre d'onde au sein du milieu de propagation considéré $\left(\mathrm{m}^{-1}\right)$. Quantitativement ce nombre représente la somme de trois composantes suivant les trois dimensions de l'espace.

$$
k^{2}=k_{x}^{2}+k_{y}^{2}+k_{z}^{2}
$$

$k_{\mathrm{c}}$ est le nombre d'onde à la fréquence de coupure du guide.

La composante $k_{z}$ est le nombre d'onde dans la direction principale de propagation de l'onde plane (assimilé à la constante de propagation guidée), donc $k_{z}^{2}=\gamma^{2}$

$$
k^{2}=k_{x}^{2}+k_{y}^{2}+\gamma^{2}
$$

avec $k_{c}^{2}=k^{2}-\gamma^{2}$

En conséquence :

$$
k_{c}^{2}=k_{x}^{2}+k_{y}^{2}
$$

Avec :

$$
k=\omega \sqrt{\mu \varepsilon}=\frac{2 \pi}{\lambda}
$$

Le nombre d'onde à la fréquence de coupure $k_{c}$ est fonction de la dimension géométrique des grands et petits côtés du guide d'ondes, tel que :

$$
\left(k_{c}\right)_{m n}=\frac{2 \pi}{\left(\lambda_{c}\right)_{m n}}=\frac{2 \pi}{\sqrt{\left(\frac{m}{a}\right)^{2}+\left(\frac{n}{b}\right)^{2}}}=\pi \sqrt{\left(\frac{m}{a}\right)^{2}+\left(\frac{n}{b}\right)^{2}}=\sqrt{\left(\frac{m \pi}{a}\right)^{2}+\left(\frac{n \pi}{b}\right)^{2}}
$$


Où $m$ et $n$ représentent le nombre de demi-sinusoides dans le sens des grands et petits côtés du guide, respectivement.

Compte tenu de la définition de la constante de propagation dans un guide d'ondes à vide :

$$
\gamma=\sqrt{k^{2}-k_{c}^{2}}
$$

Trois cas peuvent de présenter :

- Si $k^{2}>k_{c}^{2}$ : la constante de propagation $\gamma$ est un nombre réel. En conséquence la fréquence de l'onde est supérieure à la fréquence de coupure du guide et le guide d'ondes agit comme un filtre passe-haut tel que $f>\left(f_{c}\right)_{m n}$. Les ondes électromagnétiques se propagent donc dans le guide sans subir d'atténuation (Sadot et al, 2018). Pour une fréquence $f$ donnée, seuls les modes de propagation $\mathrm{TE}_{\mathrm{mn}}$ dont la fréquence est supérieure à la fréquence de coupure peuvent alors se propager.

Chaque mode ou plutôt chaque combinaison de couples $m$ et $n$, dispose de sa propre fréquence de coupure, telle que :

$$
\begin{aligned}
& \left(f_{c}\right)_{m n}=\frac{C}{\left(\lambda_{c}\right)_{m n}} \text { avec : }\left(\lambda_{c}\right)_{m n}=\frac{2 \pi}{\left(k_{c}\right)_{m n}}=\frac{2 \pi}{\pi \sqrt{\left(\frac{m}{a}\right)^{2}+\left(\frac{n}{b}\right)^{2}}}=\frac{2}{\sqrt{\left(\frac{m}{a}\right)^{2}+\left(\frac{n}{b}\right)^{2}}} \\
& \Leftrightarrow\left(f_{c}\right)_{m n}=\frac{C}{\frac{2 \pi}{\left(k_{c}\right)_{m n}}}=\frac{C}{2 \pi}\left(k_{c}\right)_{m n}=\frac{C}{2 \pi} \pi \sqrt{\left(\frac{m}{a}\right)^{2}+\left(\frac{n}{b}\right)^{2}} \\
& \Leftrightarrow\left(f_{c}\right)_{m n}=\frac{C}{2} \sqrt{\left(\frac{m}{a}\right)^{2}+\left(\frac{n}{b}\right)^{2}} \\
& \text { ou }\left(f_{c}\right)_{m n}=\frac{1}{2 \pi} \frac{1}{\sqrt{\mu \varepsilon}} \sqrt{\left(\frac{m \pi}{a}\right)^{2}+\left(\frac{n \pi}{b}\right)^{2}} \text { avec : } C=\frac{1}{\sqrt{\mu \varepsilon}}
\end{aligned}
$$

Le mode avec la fréquence de coupure la plus basse, est appelé mode dominant ou mode fondamental. Dans le cas où les dimensions du guide d'ondes respectent la condition $a>b$, la fréquence de coupure la plus basse est rencontrée dans le cas où $\mathrm{m}=1$ et $\mathrm{n}=0$ (mode TE10).

De même, la longueur d'onde de coupure dans un guide vide pour un mode fondamental $\mathrm{TE}_{m n}$ est donnée par la relation suivante :

$$
\left(\lambda_{c}\right)_{m n}=\frac{2}{\sqrt{\left(\frac{m}{a}\right)^{2}+\left(\frac{n}{b}\right)^{2}}}
$$

Dans le cas du mode TE10, la longueur d'onde de coupure est égale à

$$
\left(\lambda_{c}\right)_{10}=\frac{2}{\sqrt{\left(\frac{1}{a}\right)^{2}+\left(\frac{0}{b}\right)^{2}}}=2 a
$$


Pour le mode TE10, l'onde se propage à la condition où l'inégalité suivante est respectée :

$$
f>\frac{C}{\left(\lambda_{c}\right)_{10}}=\frac{C}{2 a}, \text { soit : } a>\frac{C}{2 f}
$$

Pour $\mathrm{f}=2,45 \mathrm{GHz}$ :

$$
a>\frac{3 \times 10^{8}}{2 \times 2.45 \times 10^{9}}=0,0612 \mathrm{~m}
$$

La longueur du grand côté d'un guide d'ondes en mode TE10 est de 0,086 m, donc la condition de propagation est bien respectée.

La longueur d'onde guidée est quant à elle définie comme la distance entre deux crêtes tout au long de l'axe de propagation.

$$
\left(\lambda_{g}\right)_{m n}=\frac{2 \pi}{\gamma}=\frac{2 \pi}{\sqrt{k^{2}-k_{c}^{2}}}=\frac{2 \pi}{\sqrt{\omega^{2} \mu \varepsilon-\left(\frac{m \pi}{a}\right)^{2}-\left(\frac{n \pi}{b}\right)^{2}}}
$$

$\left(\lambda_{g}\right)_{m n}$ est généralement plus grand que la longueur d'ondes en espace libre $\lambda=\frac{2 \pi}{k}$ car $\gamma^{2}<k^{2}$.

La célérité de l'onde dans le guide est définie par le rapport entre la pulsation $\omega$, et la constante de propagation de cette onde.

$$
v_{p}=\frac{\omega}{\gamma}>\frac{\omega}{k}=\frac{1}{\sqrt{\mu \varepsilon}}=C
$$

Cas particulier :

Dans le cas où $\left(f_{c}\right)_{10}<f<\left(f_{c}\right)_{20}$ : la longueur d'onde en espace libre est inférieure à la longueur d'onde de coupure et le guide ne laisse passer que le mode fondamental $\mathrm{TE}_{10}$. L'onde subit alors une atténuation minimale et se propage dans le guide.

Dans le cas où $f>\left(f_{c}\right)_{20}$ : plusieurs modes peuvent se propager simultanément avec leur propre distribution de champs et longueur d'onde de propagation.

- $\mathrm{Si} k^{2}=k_{c}^{2}$ : La constante de propagation longitudinale est nulle, il n'y a pas de propagation ou d'atténuation selon l'axe de propagation $z$. La propagation est seulement présente sur le plan transverse du guide.

- Si $k^{2}<k_{c}^{2}$ : la constante de propagation est un nombre complexe. Dans ce cas, la fréquence de l'onde est inférieure à la fréquence de coupure du guide $\left(f<\left(f_{c}\right)_{m n}\right)$. L'onde est dite évanescente, il n'y a pas de propagation. Le résultat essentiel est que pour toute fréquence d'excitation inférieure à la fréquence de coupure d'un guide vide, la propagation des ondes est impossible.

Les modes caractérisés par une fréquence inférieure à la fréquence de coupure conduisent à une constante de propagation $\gamma$ imaginaire. En conséquence toutes les composantes du champ électrique sont considérées comme exponentielles décroissantes en fonction de la distance à la source 
d'excitation. Ces modes sont plus communément appelés modes «sous coupure» ou modes évanescents.

Compte tenu du mode de propagation de l'onde dans un guide (mode fondamental), il est possible de réaliser des fentes au niveau des parois du guide à la seule condition de ne pas couper les lignes de courant au niveau des parois du guide (sous peine de provoquer une propagation par la fente). Physiquement, le comportement de l'onde dépend de l'intensité et du signe des lignes de courants " coupées » par la fente. Ces lignes de courant sur les parois du guide sont représentées sur la figure 3.5 pour un guide d'ondes en mode fondamental TE10. Différents types de fentes plus ou moins rayonnantes y sont illustrées.

Le comportement rayonnant ou non de la fente est fonction de sa position sur le guide. Afin de prédire le calcul de l'interaction de la fente avec le guide, il est nécessaire de mener des simulations numériques car le phénomène dépend de la fréquence et de la configuration des fentes au niveau des parois.

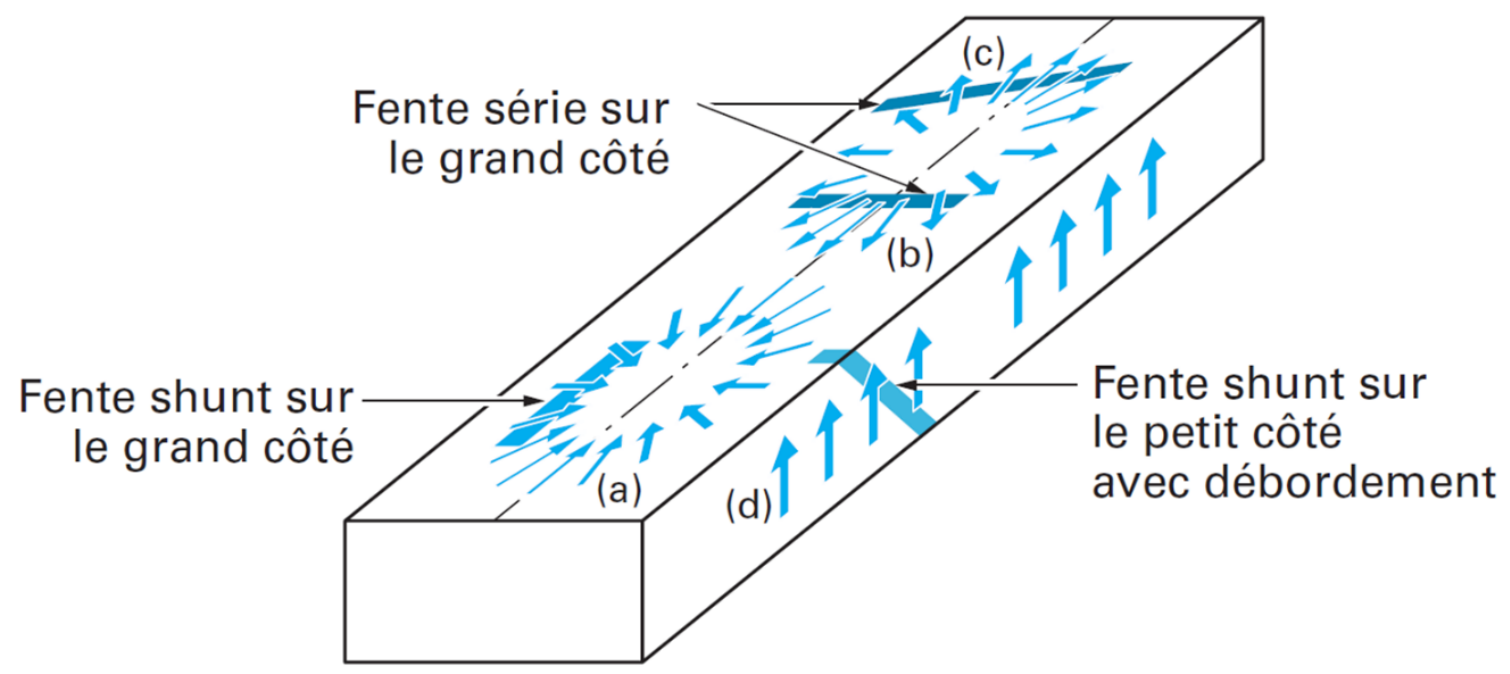

Les flèches $(\rightarrow)$ schématisent les courants sur les parois du guide

Figure 3.5. Quelques exemples de fentes sur un guide rectangulaire (d'après Debionne, 2005)

Il convient de noter que suivant le type de fréquence micro-ondes considéré ( $915 \mathrm{MHz}$ ou 2450 $\mathrm{MHz}$ ), les dimensions du guide d'ondes ( $a$ et $b$ ) peuvent varier. Ceci impact donc les modes de propagation qui peuvent exister dans chaque structure guidée.

Dans le cas particulier où $f>\left(f_{c}\right)_{10}$, on distingue deux propagations majeurs des ondes électromagnétique au sein d'un guide d'ondes : les ondes progressives et les ondes stationnaires.

\subsection{Les ondes progressives}

Dans le cas d'une onde progressive, le champ électrique est considéré constant tout au long de l'axe de propagation du guide d'ondes (norme du champ électrique). Dans ce cas particulier, l'extrémité du guide d'ondes est terminée par une charge diélectrique absorbante sans réflexion (eau dans la majeure partie des cas). Cette charge à eau est dimensionnée pour absorber la totalité de l'énergie de l'onde résiduelle. 


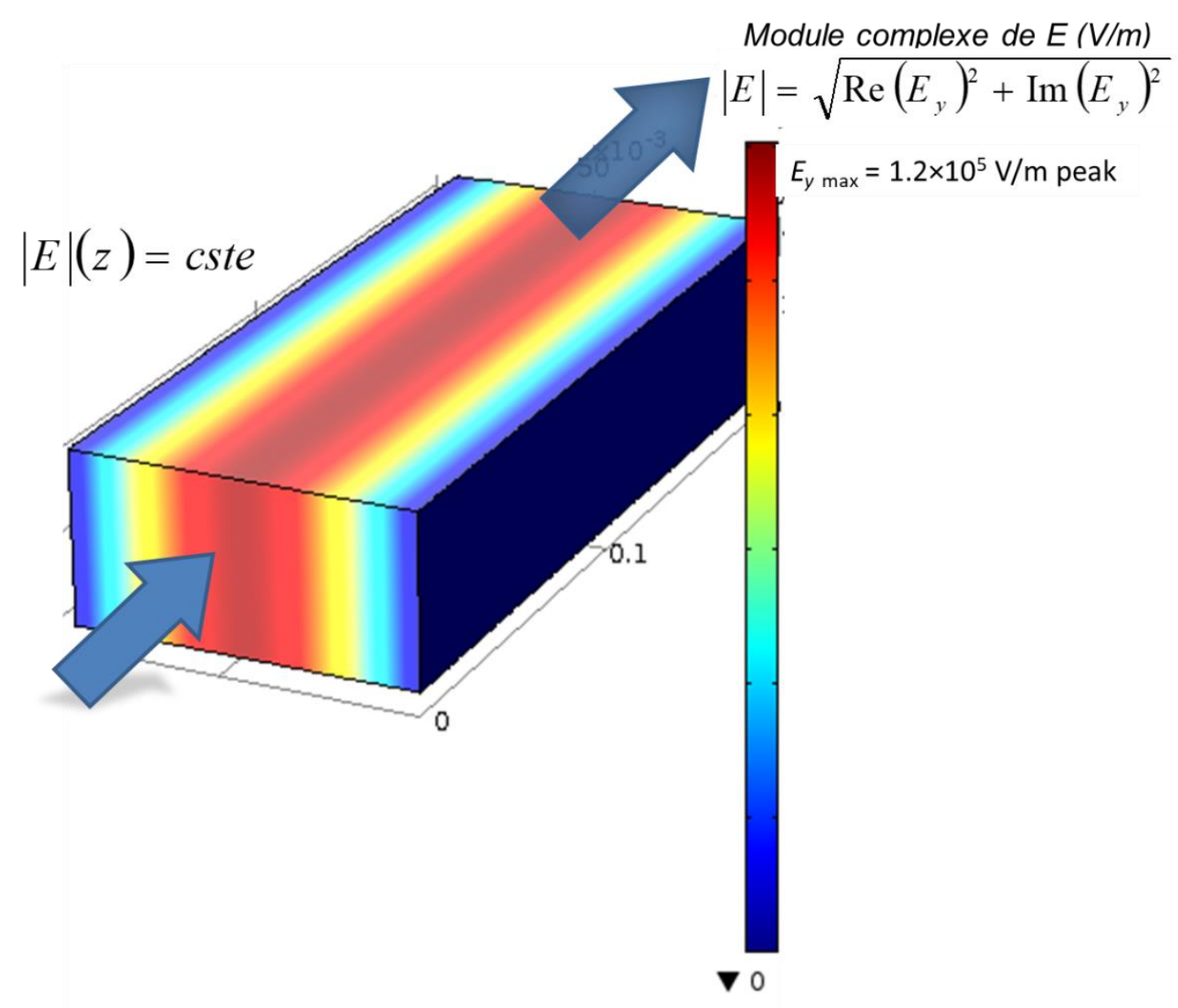

Figure 3.6. Forme du champ électrique (norme) dans un guide d'ondes vide soumis à une onde progressive en mode TE10 $(f=2450 \mathrm{MHz}$, Pin $=25 \mathrm{~kW})$

La figure 3.6 illustre la norme (ou module complexe) du champ électrique au sein d'un guide d'ondes rectangulaire TE10 pour une fréquence $f=2450 \mathrm{MHz}$, et pour une puissance incidente de $25 \mathrm{~kW}$. Le régime d'ondes progressives se caractérise dans ce cas par un champ électrique (norme $\mathrm{E}$ suivant $z$ ) constant suivant l'axe de propagation de l'onde et qui varie selon l'axe $x$.

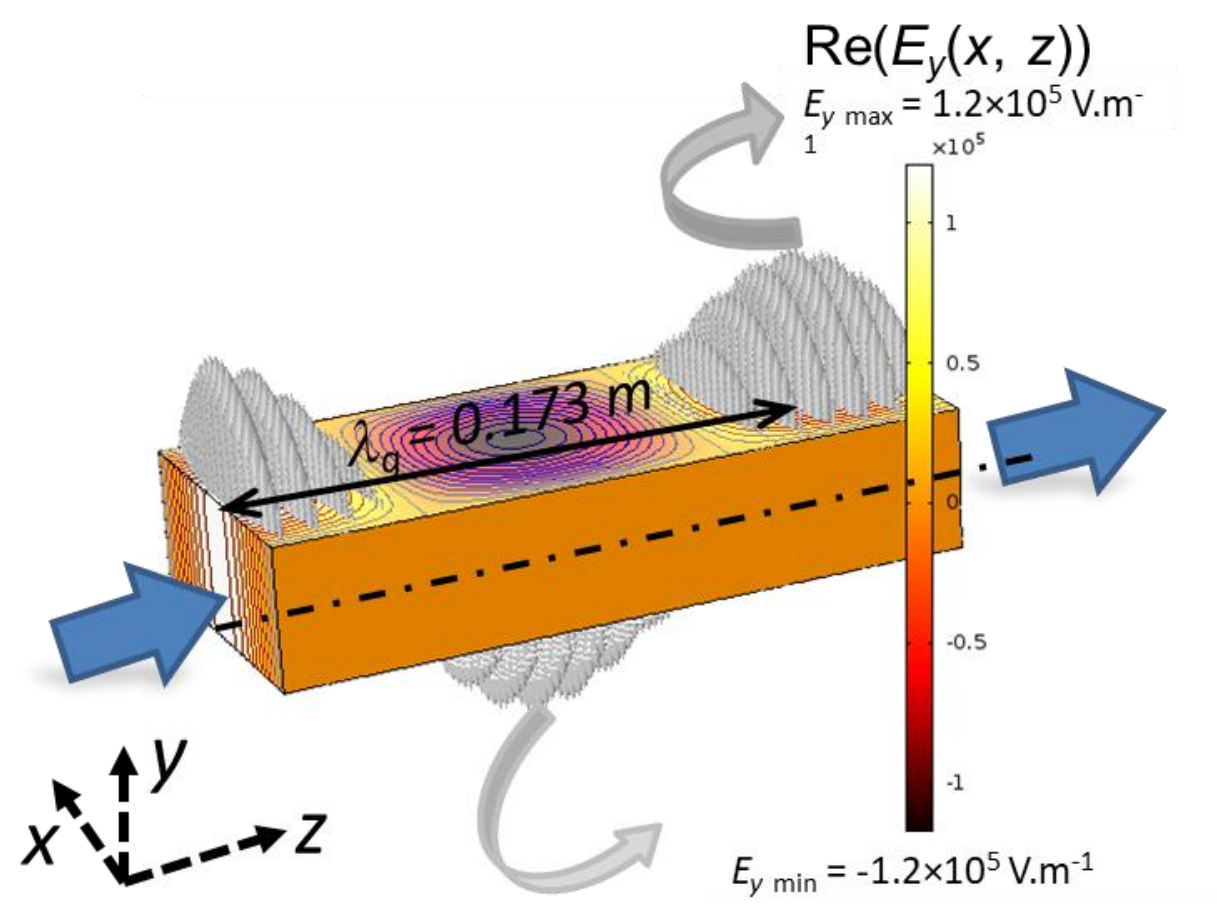

Figure 3.7. Distribution du champ électrique (partie réelle de la composante Ey) dans un guide d'ondes vide soumis à une onde progressive en mode TE10 $(f=2450 \mathrm{MHz}$, Pin $=25 \mathrm{~kW})$ 
La figure 3.7 illustre la distribution du champ électrique (partie réelle de la composante $E_{y}$ ) au sein d'un guide d'ondes rectangulaire TE10 pour une fréquence de $2450 \mathrm{MHz}$, et pour une puissance incidente de $25 \mathrm{~kW}$. Le régime d'ondes progressives se caractérise dans ce cas par une composante $E_{y}$ du champ électrique qui varie selon les axes $\mathrm{x}$ et $\mathrm{z}$.

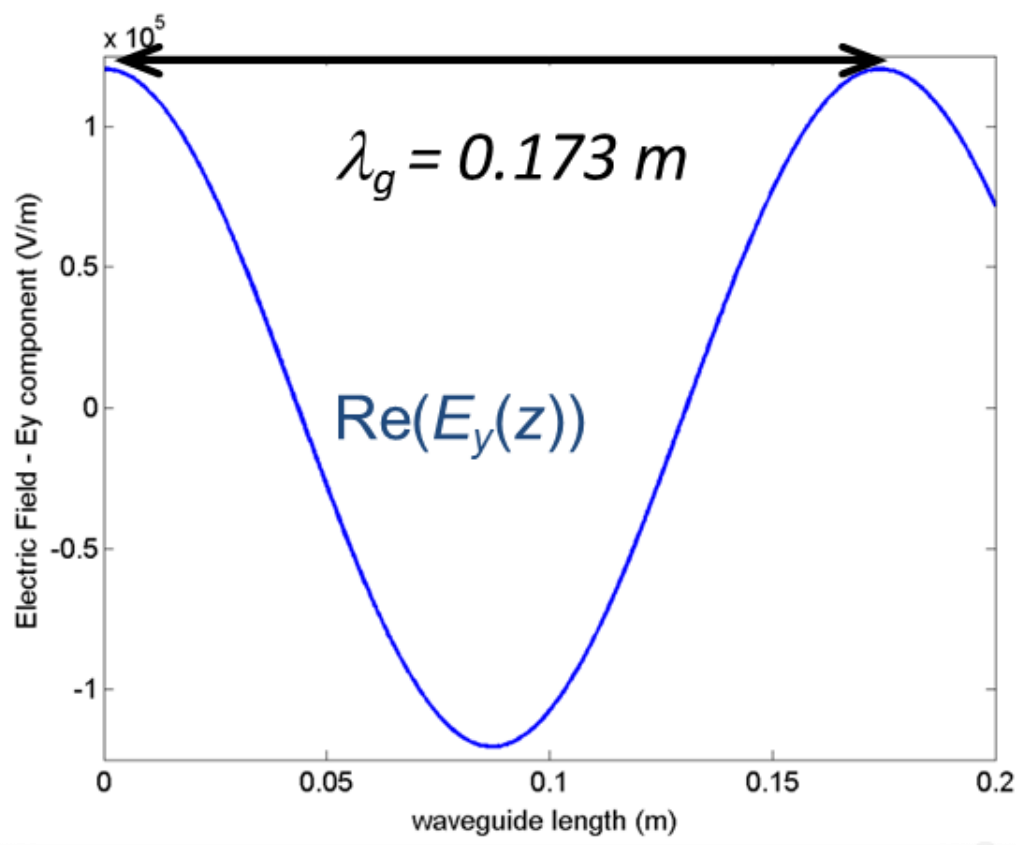

Figure 3.8. Distribution du champ électrique (partie réelle de la composante Ey) dans le cas d'une onde progressive suivant l'axe de propagation $z(f=2450 \mathrm{MHz}$, Pin $=25 \mathrm{~kW})$

La figure 3.8 illustre la forme de la partie réelle du champ électrique $E_{y}(z)$ suivant l'axe de propagation $z$. On remarque que la longueur d'onde guidée est égale à la distance entre deux maximum de $E_{y}$ des champs électriques. La distance entre deux extremum de $E_{y}$ correspond à la moitié de la longueur d'onde guidée (ici $0,173 \mathrm{~m}$ à la fréquence de $2,45 \mathrm{GHz}$ ).

On définit le taux d'onde stationnaire (TOS) par le rapport entre les valeurs absolues des champs électriques $E_{\max }$ et $E_{\min }$ dans le guide.

$$
\operatorname{TOS}=\frac{\left|E_{\max }\right|}{\left|E_{\min }\right|}
$$

Pour une onde progressive, ce taux d'ondes stationnaires est égal à 1. Le mode de propagation en ondes progressive reste malgré tout peu utilisé pour des applications industrielles car ce mode ne permet pas toujours d'optimiser le transfert d'énergie vers la charge diélectrique (puissance réfléchie par la charge diélectrique placée dans le guide d'ondes).

\subsection{Les ondes stationnaires}

Afin de maximiser le transfert de l'énergie contenue dans l'onde électromagnétique vers le produit à traiter, on préfère utiliser un mode de progagation en ondes stationnaires. Dans ce cas particulier, l'extrémité du guide d'ondes est terminée par une plaque métallique qui réfléchie les ondes dans la cavité formée. 
Dans le cas d'une onde stationnaire, les champs électromagnétiques sont caractérisés par des ventres et des nœuds tout au long de l'axe de propagation des ondes. Il en résulte en quelque sorte une superposition multiple des ondes incidentes et réfléchies dans le guide (Sadot et al. 2018). Les maximums de champ électrique sont alors séparés par une demi-longueur d'ondes guidée (séparation des extremums de $\lambda_{g} / 4$ ) (figure 3.9).

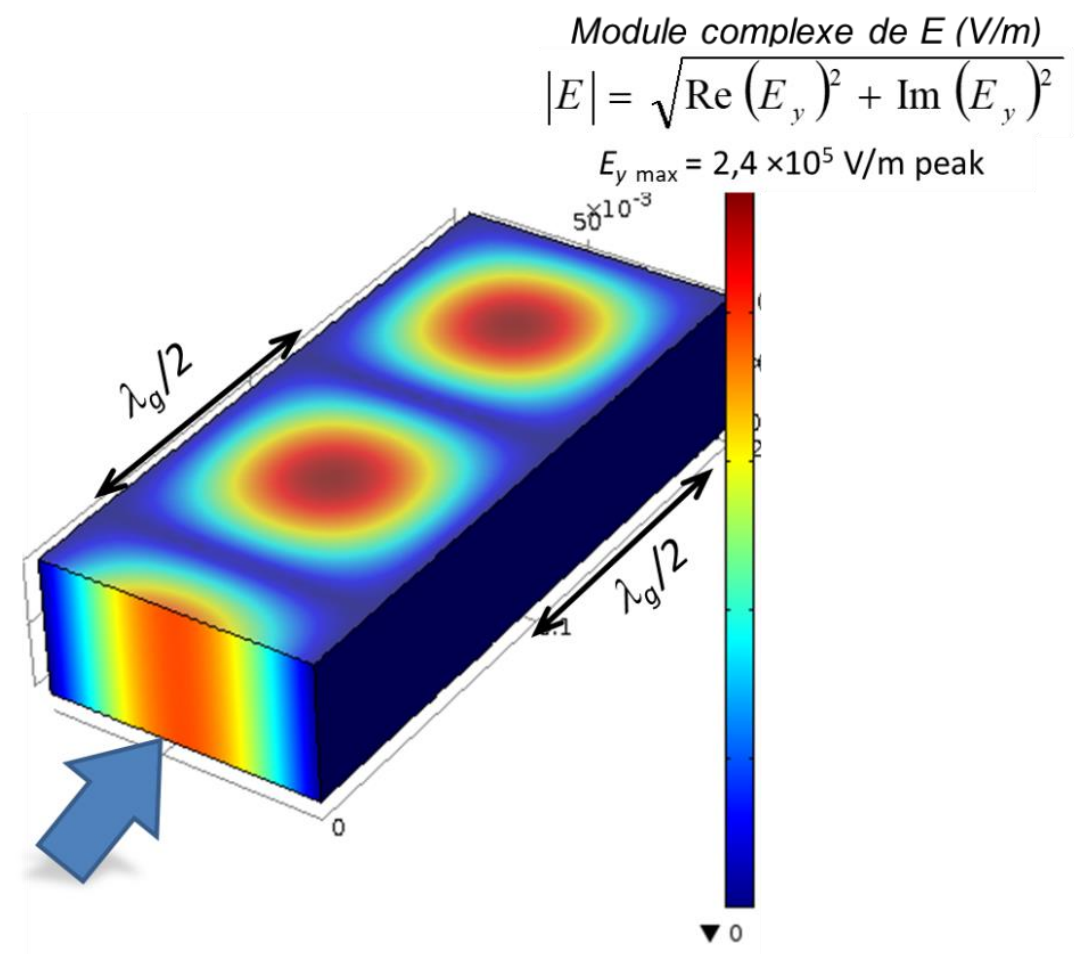

Figure 3.9. Forme du champ électrique (norme) dans un guide d'ondes vide soumis à une onde stationnaire en mode TE10 $(f=2450 \mathrm{MHz}$, Pin $=25 \mathrm{~kW})$

La figure 3.9 illustre la norme (ou module complexe) du champ électrique au sein d'un guide d'ondes rectangulaire TE10 pour une fréquence de $2450 \mathrm{MHz}$, et pour une puissance incidente de $25 \mathrm{~kW}$. Le régime d'ondes stationnaires se caractérise dans ce cas par un champ électrique (norme $\mathrm{E}$ suivant l'axe $z$ ) avec des ventres et des nœuds suivant l'axe de propagation de l'onde et qui varie selon l'axe $\mathrm{x}$. 


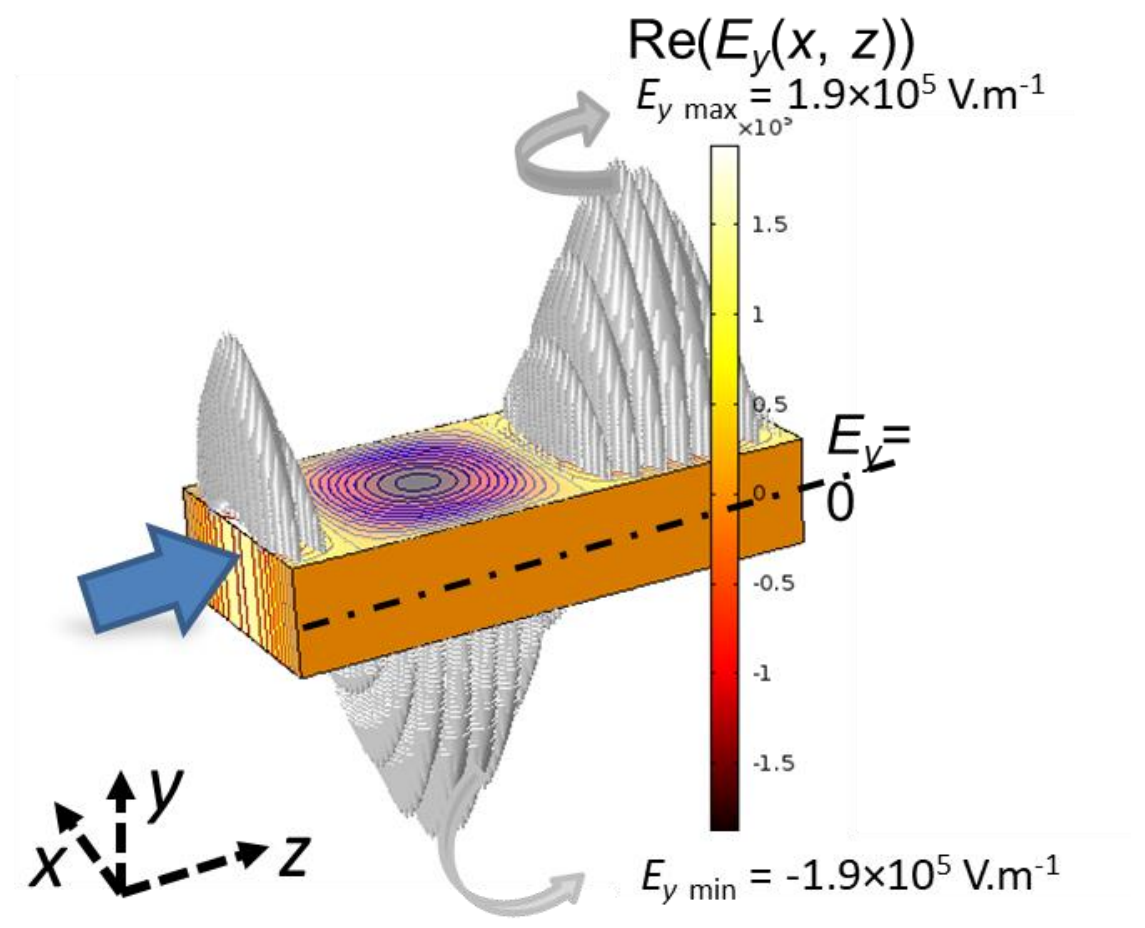

Figure 3.10. Distribution du champ électrique (partie réelle de la composante Ey) dans un guide d'ondes vide soumis à une onde stationnaire en mode TE10 $(f=2450 \mathrm{MHz}$, Pin $=25 \mathrm{~kW})$

La figure 3.10 illustre la distribution du champ électrique (partie réelle de la composante $E_{y}$ ) au sein d'un guide d'ondes rectangulaire TE10 pour une fréquence $2450 \mathrm{MHz}$, et pour une puissance incidente de $25 \mathrm{~kW}$. Le régime d'ondes stationnaires se caractérise dans ce cas par une composante $E_{y}$ du champ électrique qui varie selon les axes $\mathrm{x}$ et $\mathrm{z}$.

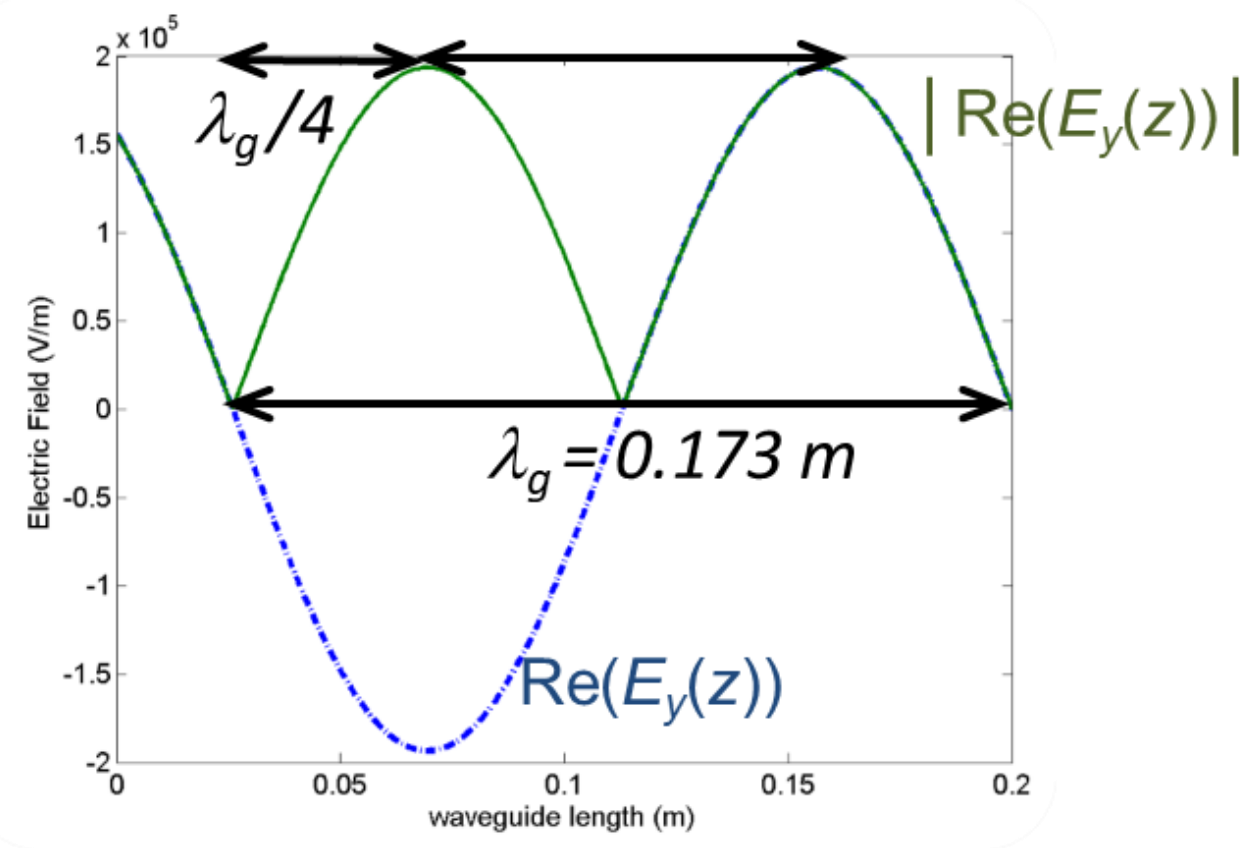

Figure 3.11. Distribution du champ électrique (partie réelle de la composante Ey) dans le cas d'une onde stationnaire suivant l'axe de propagation z $(f=2450 \mathrm{MHz}$, Pin $=25 \mathrm{~kW})$ 
La figure 3.11 illustre la forme de la partie réelle du champ électrique $E_{y}(z)$ suivant l'axe de propagation $z$. On remarque que la longueur d'onde guidée est égale à deux fois la période du champ électrique $E_{y}$. De même, la distance entre deux valeurs absolues des extremum $E_{y}$ max et $E_{y}$ min correspond au quart de la longueur d'onde guidée.

Dans le cas d'une onde stationnaire, la valeur du taux d'ondes stationnaires (TOS) peut être supérieure à 1 .

\subsection{Cas des interactions avec une charge diélectrique}

La configuration en guide d'ondes permet de positionner un produit à traiter entre l'entrée du guide et la sortie (figure 3.12). Si l'extrémité du guide est terminé par une charge absorbante, l'onde incidente est partiellement réfléchie à l'interface air-produit en fonction des valeurs des propriétés diélectriques (configuration en onde progressive). Suivant la taille et les caractéristiques diélectriques du produit, l'onde sera plus ou moins réfléchie, absorbée ou transmise.

On distingue majoritairement deux régimes permettant de prédire la forme du champ électrique et la génératino de chaleur dans le produit traité en fonction de la taille des échantillons et de la profondeur de pénétration des ondes : le régime de décroissance exponentielle et le régime résonnant.

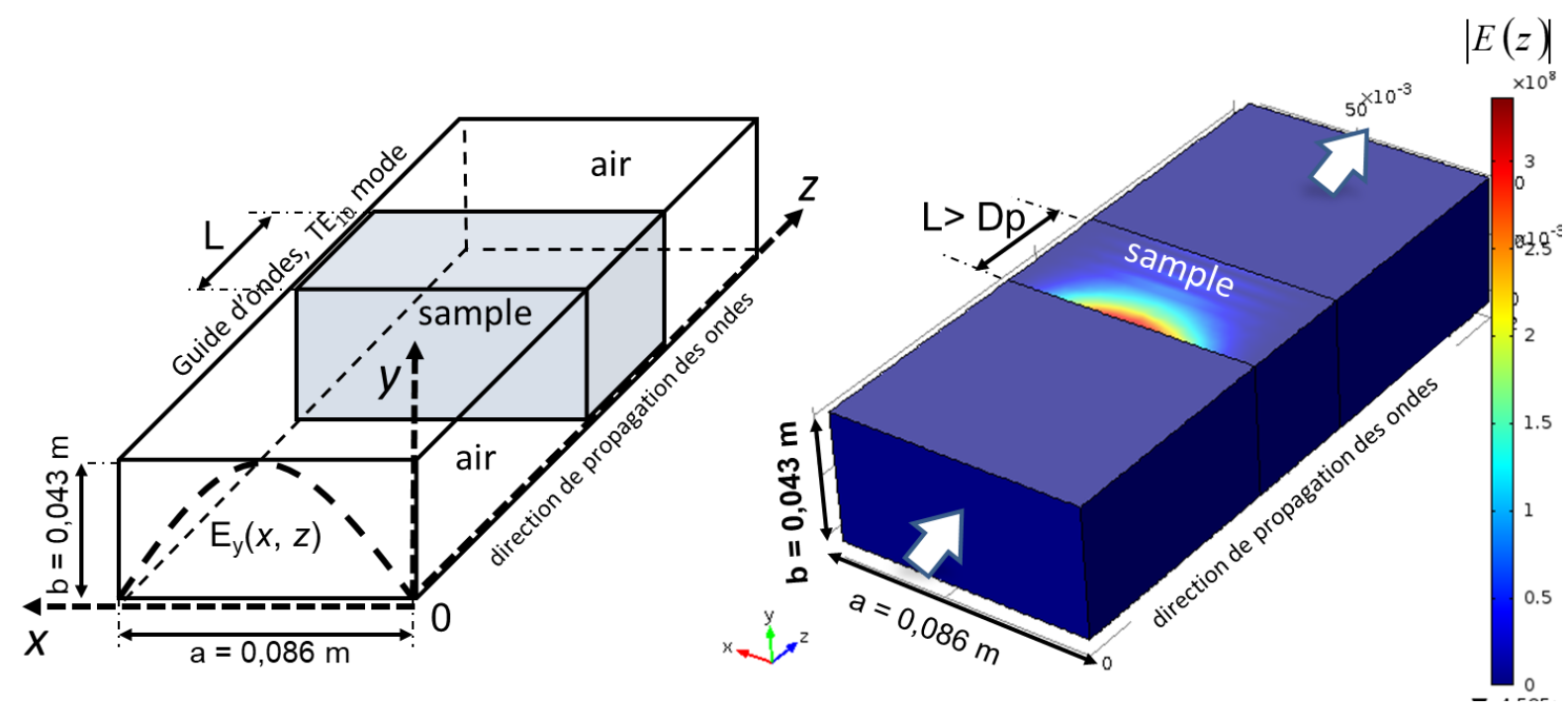

Figure 3.12. Forme du champ électrique (norme) dans un guide d'ondes rempli d'un produit dans sa section et soumis à une onde progressive en mode TE10 $(f=2450 \mathrm{MHz}, \operatorname{Pin}=25 \mathrm{~kW})$

Dans l'exemple de la figure 3.12, le champ électrique dans le produit suit une décroissance exponentielle en fonction de $z$ par rapport à la surface. Dans le cas particulier où l'épaisseur du produit (ici, $L$ ) est importante face à la profondeur de pénétration des ondes (ici, $D_{p}$ ) dans le produit, le champ électrique décroit habituellement de manière exponentielle en fonction de la profondeur dans le produit (plus communément désigné sous le nom de loi de Lambert).

La figure 3.13 illustre la propagation monodimensionnelle (1D) d'une onde électromagnétique plane au sein d'un produit dont l'épaisseur (ici, $e_{p}$ ) est très importante par rapport à la profondeur de pénétration. Le produit est entouré par de l'air uniquement à la surface supérieure. Il repose sur un support transparent au rayonnement micro-ondes à la surface inférieure (diélectrique parfait). L'onde incidente est caractérisée par un champ électrique incident constant $E_{0}$ associé à un flux de puissance micro-onde $F_{0}$. 


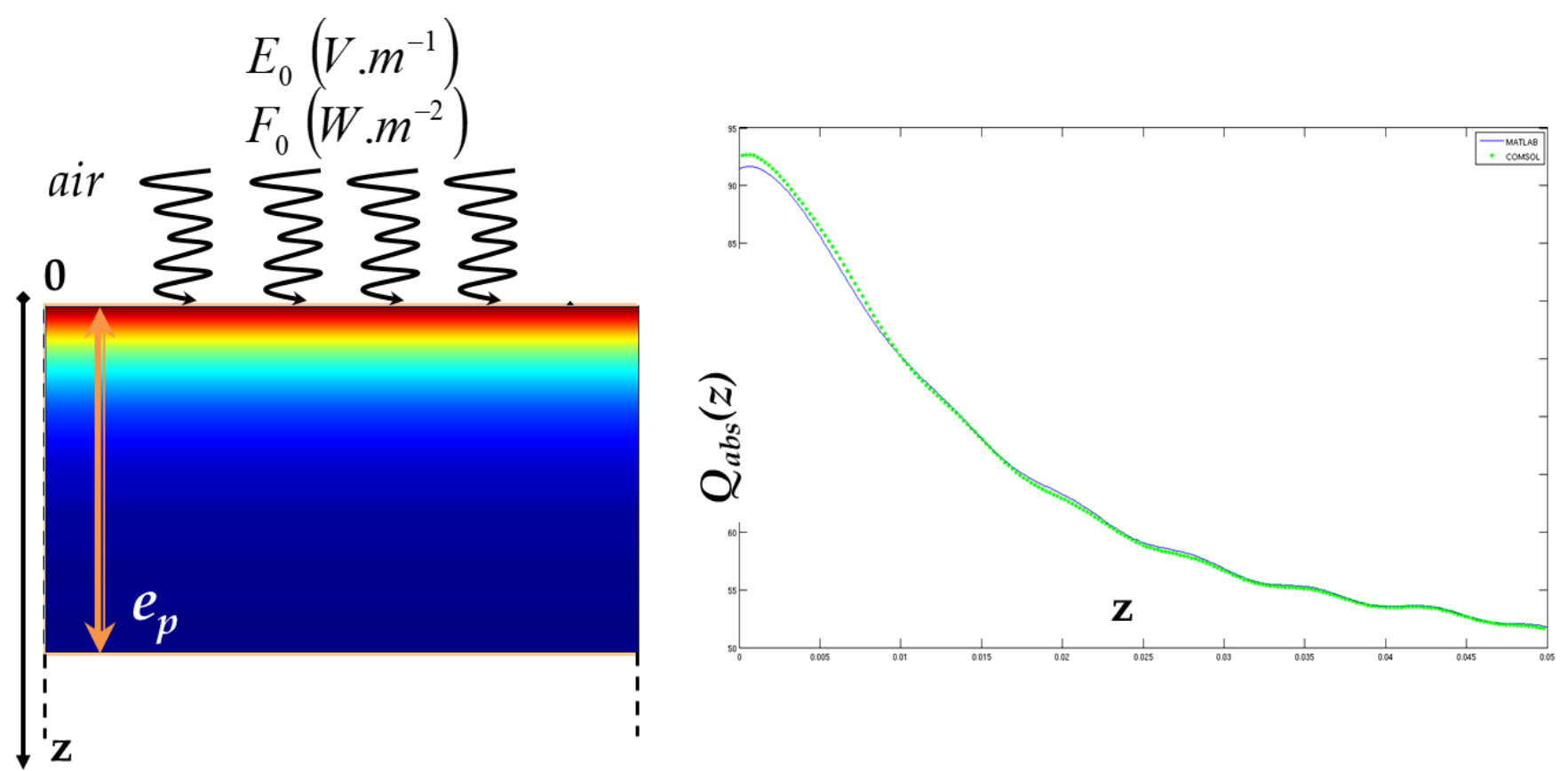

Figure 3.13. Propagation 1D d'une onde électromagnétique plane au sein d'un produit par la surface supérieure : cas particulier de la loi de Lambert

Dans ce cas, la puissance micro-ondes absorbée par unité de volume dans le produit suit une décroissance exponentielle à partir de la surface du produit en fonction de l'axe $z$, En fonction de l'ordre de grandeur de l'épaisseur du produit par rapport à la profondeur de pénétration, on peut aussi observer la présence de légères oscillations lors de la décroissance exponentielle. Ceci est dû à un régime de résonance qui peut venir se superposer à la décroissance exponentielle.

La figure 3.14 illustre la propagation monodimensionnelle (1D) d'une onde électromagnétique plane au sein d'un produit dont l'épaisseur $\left(e_{p}\right)$ est inférieure ou proche de la profondeur de pénétration des ondes. Le produit est entouré par de l'air uniquement à la surface supérieure. Il repose sur un support transparent au rayonnement micro-ondes à la surface inférieure (diélectrique parfait). L'onde incidente est caractérisée par un champ électrique incident constant $E_{0}$ associé à un flux de puissance microondes $F_{0}$. 


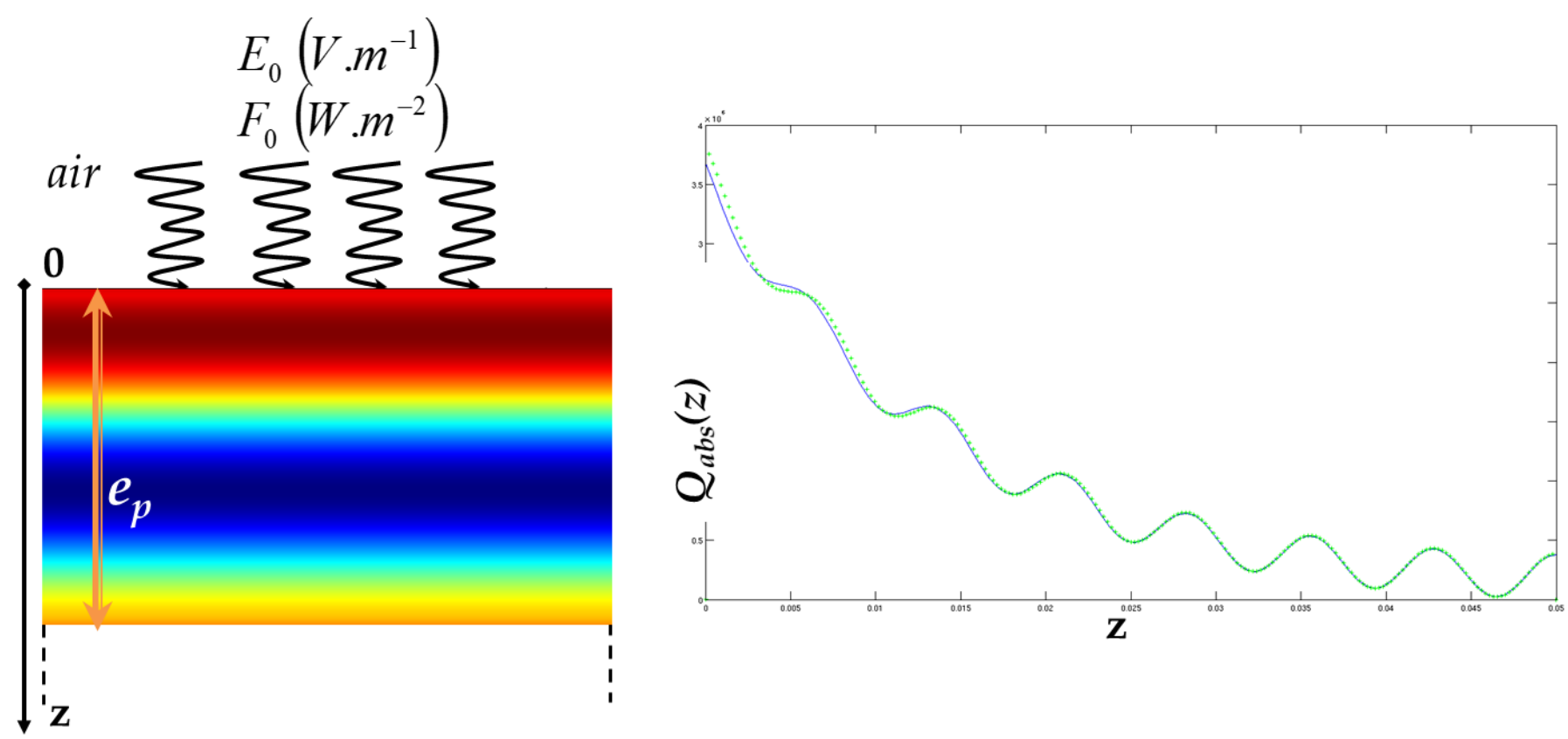

Figure 3.14. Propagation 1D d'une onde électromagnétique plane au sein d'un produit par la surface supérieure : cas particulier du régime résonnant

Dans ce cas particulier, la puissance micro-ondes absorbée par unité de volume dans le produit ne suit plus une décroissance exponentielle en fonction de l'axe $z$, mais présente un comportement ondulatoire d'amplitude variable en fonction des propriétés diélectriques du produit. Ce comportement est plus communément désigné par le régime de résonnance et peut conduire à la présence de points chauds localement au sein du produit.

\section{Dispositifs expérimentaux}

Dans le cas d'un dispositif de traitement micro-ondes classique dans un four micro-ondes ménager, l'inconvénient majeur réside dans la difficulté de prédire la forme du champ électrique dans la cavité (cas de la propagation multimode). Pour palier à ce problème, on préfère utiliser des cavités microondes monomode qui présentent l'avantage de disposer d'une forme des champs électriques incidents mieux maîtrisée. Ceci permet aussi de faciliter la modélisation du dispositif pour mieux comprendre les interactions micro-ondes-matière et proposer des solutions d'optimisation.

La figure 4.1 suivante présente de manière schématique quelques éléments constituants une cavité micro-ondes monomode avec propagation d'ondes stationnaires en guide d'ondes rectangulaire. 


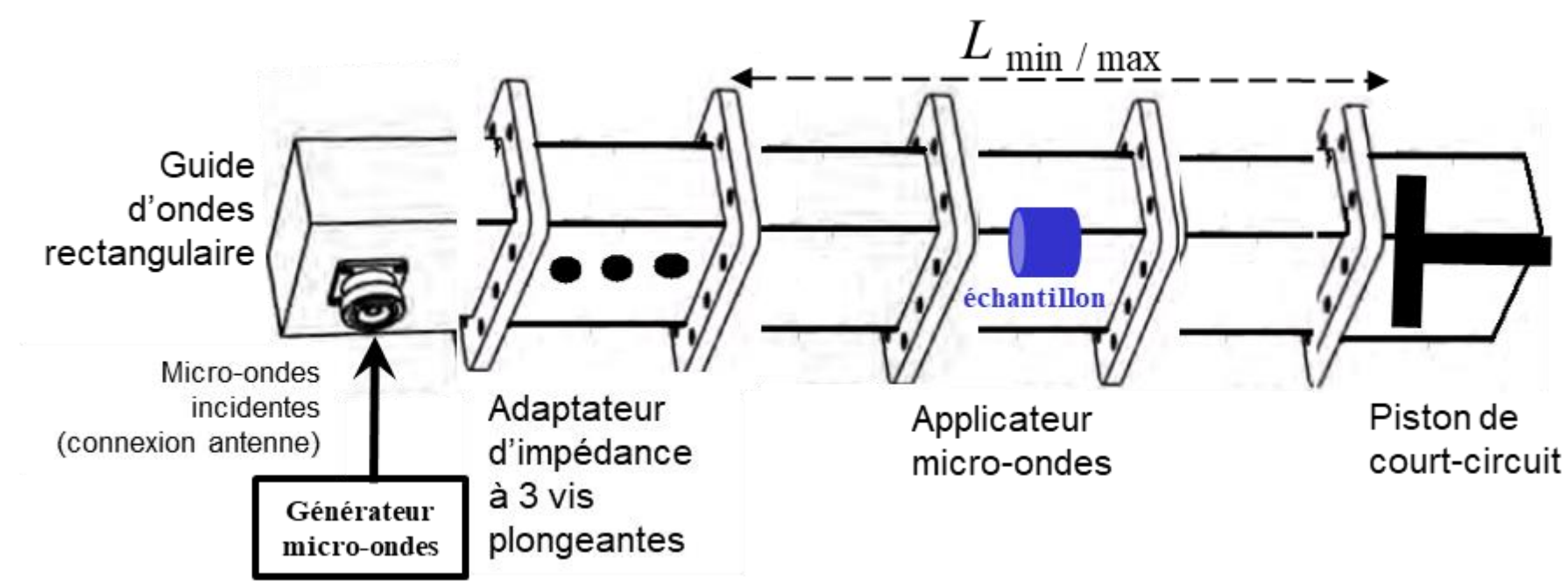

Figure 4.1. Représentation schématique des éléments constituants une cavité micro-ondes monomode avec propagation en ondes stationnaires

\subsection{Les générateurs micro-ondes}

Les dispositifs micro-ondes dédiés à la recherche et développement sont la plupart du temps constitués d'un générateur micro-ondes à puissance variable. Ce générateur peut être soit constitué de magnétrons ou à base de semi-conducteurs (transistors). On préférera utiliser ces derniers dits à « état solide » car ils possèdent un spectre de fréquence précis et modulable avec précision (par exemple dans la gamme $2450 \mathrm{MHz} \pm 50 \mathrm{MHz}$ ), comme c'est le cas dans les applications majeures rencontrées en chauffage micro-ondes.

A la sortie du générateur micro-ondes, plusieurs connexions peuvent être rencontrées pour alimenter le guide d'ondes rectangulaire en puissance. Habituellement, les générateurs disposent soit d'une connexion via câble coaxial permettant de relier le générateur à l'entrée du guide d'ondes (transition guide-coaxial). Le deuxième cas rencontré concerne une connexion directe entre le générateur et le guide d'ondes sans câble coaxial (figure 4.2). Cette solution permet principalement de limiter les pertes de puissances micro-ondes qui seraient rencontrées dans un câble coaxial de longueur importante avec des connecteurs intermédiaires au niveau de la transition guide-coaxial. 


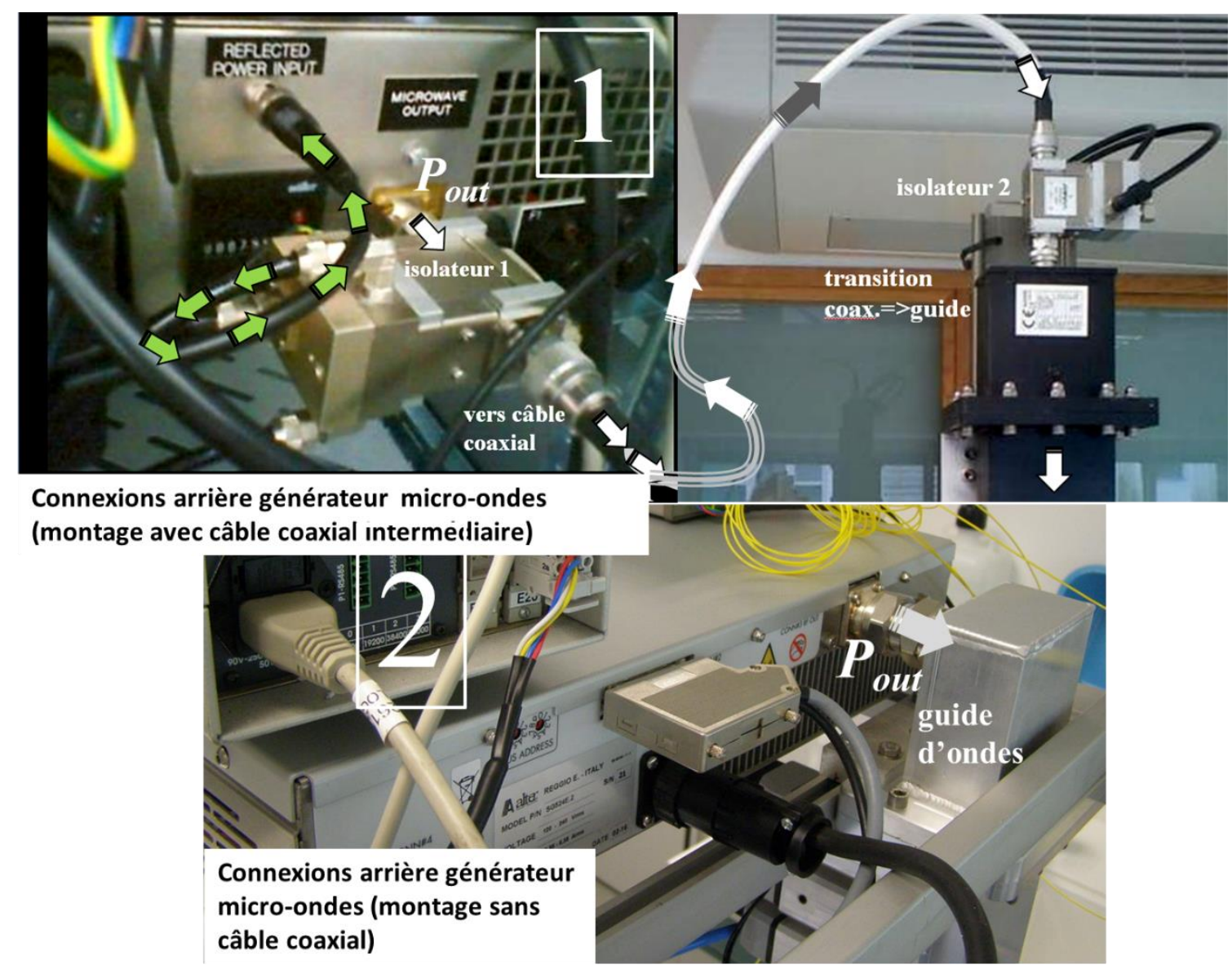

Figure 4.2. Montage avec ou sans câble coaxial pour transmission de puissance micro-ondes

\subsection{L'applicateur}

Dans le cas d'un traitement de produits alimentaires par micro-ondes, la conception de l'applicateur qui contient l'échantillon est une phase primordiale sur laquelle il faut particulièrement s'attarder. Plusieurs sources bibliographiques insistent sur l'importance de la conception de l'applicateur microondes car cela conditionne toute l'efficacité du traitement thermique sur le produit (Luan et al, 2016).

Comme illustré sur la figure 4.3, ces applicateurs sont positionnés la plupart du temps entre la source micro-ondes et l'extrémité du guide d'ondes (charge absorbante ou court-circuit suivant si l'on considère une onde progressive ou stationnaire). 


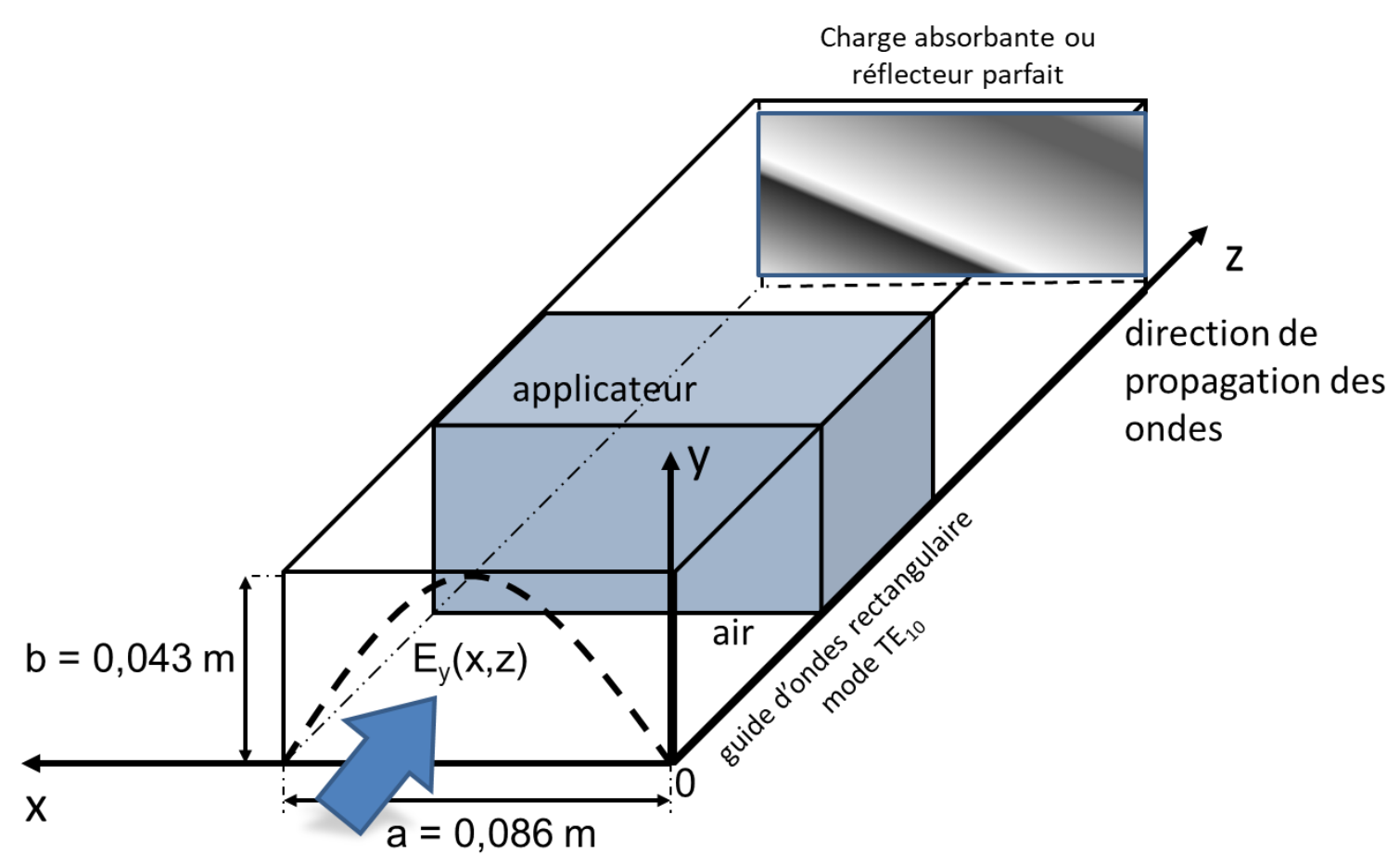

Figure 4.3. Schématisation d'un guide d'ondes monomode avec applicateur

Dans le cas d'une propagation d'ondes progressives, la puissance absorbée sur la charge en sortie de guide d'ondes est souvent mesurée par calorimétrie en fonction de la différence de température de l'eau qui la traverse. À partir de la puissance micro-ondes incidente et de la mesure de puissance réfléchie, on peut alors estimer la puissance absorbée par le produit moyennant les pertes de puissances ou niveau du guide d'ondes et des diverses connexions intermédaires le cas échéant.

Dans le cas d'une propagation d'ondes stationnaire, l'extrémité du guide est terminée par une plaque métallique qui réfléchie l'ensemble de l'onde incidente. Dans ce mode de propagation, le principe général consiste à positionner le produit dans un endroit de la cavité afin d'optimiser le rendement énergétique de la source vers la charge (adaptation d'impédance). Si une plaque métallique est positionnée au bout d'un guide vide, la puissance réfléchie doit être égale à $100 \%$. Le fait d'introduire un produit au sein du guide va modifier la propagation de l'onde stationnaire et la position des ventres et des nœuds du champ électrique suivant l'axe de propagation. Sans aucun réglage complémentaire, la puissance micro-ondes incidente ne sera jamais égale à la puissance absorbée par l'échantillon (sauf cas particulier et très aléatoire en fonction de la géométrie des produits et de leurs caractéristiques diélectriques). C'est là qu'interviennent les éléments d'adaptation d'impédance pour maximiser le rendement énergétique.

\subsection{Notion d'adaptation d'impédance}

Dans le cas d'une propagation d'ondes stationnaires, afin de maximiser le transfert d'énergie microondes vers la charge diélectrique à traiter, il est nécessaire de créer une cavité résonnante entre l'élément d'adaptation et l'extrémité du guide d'ondes (plaque métallique). La cavité résonnante permet ainsi d'imposer une forme particulière au champ électromagnétique (ondes stationnaires) afin d'accumuler de l'énergie pour chauffer de manière optimale le produit à traiter. Pour ceci, la puissance réfléchie est un paramètre important à contrôler et à minimiser au cours du procédé.

Au niveau technique, on distingue souvent deux types d'éléments d'adaptation d'impédance : les vis plongeantes (ou stubs) et les iris de couplage. Les vis plongeantes (les plus répandues) sont constituées de cylindres positionnés le long du grand côté du guide d'ondes et qui peuvent translater dans la direction transverse à la propagation des ondes dans le guide. Suivant les cas, on peut rencontrer des adaptateurs à 3 vis plongeantes ou plus. Par défaut les vis affleurent la surface du grand côté du guide 
(figure 4.4). Le fait de modifier la distance des vis par rapport à la paroi métallique du guide d'ondes permet de modifier la forme du champ électrique au sein de la cavité. Il n'existe pas de règles absolues concernant le réglage des vis plongeantes et cela résulte souvent d'une démarche empirique.

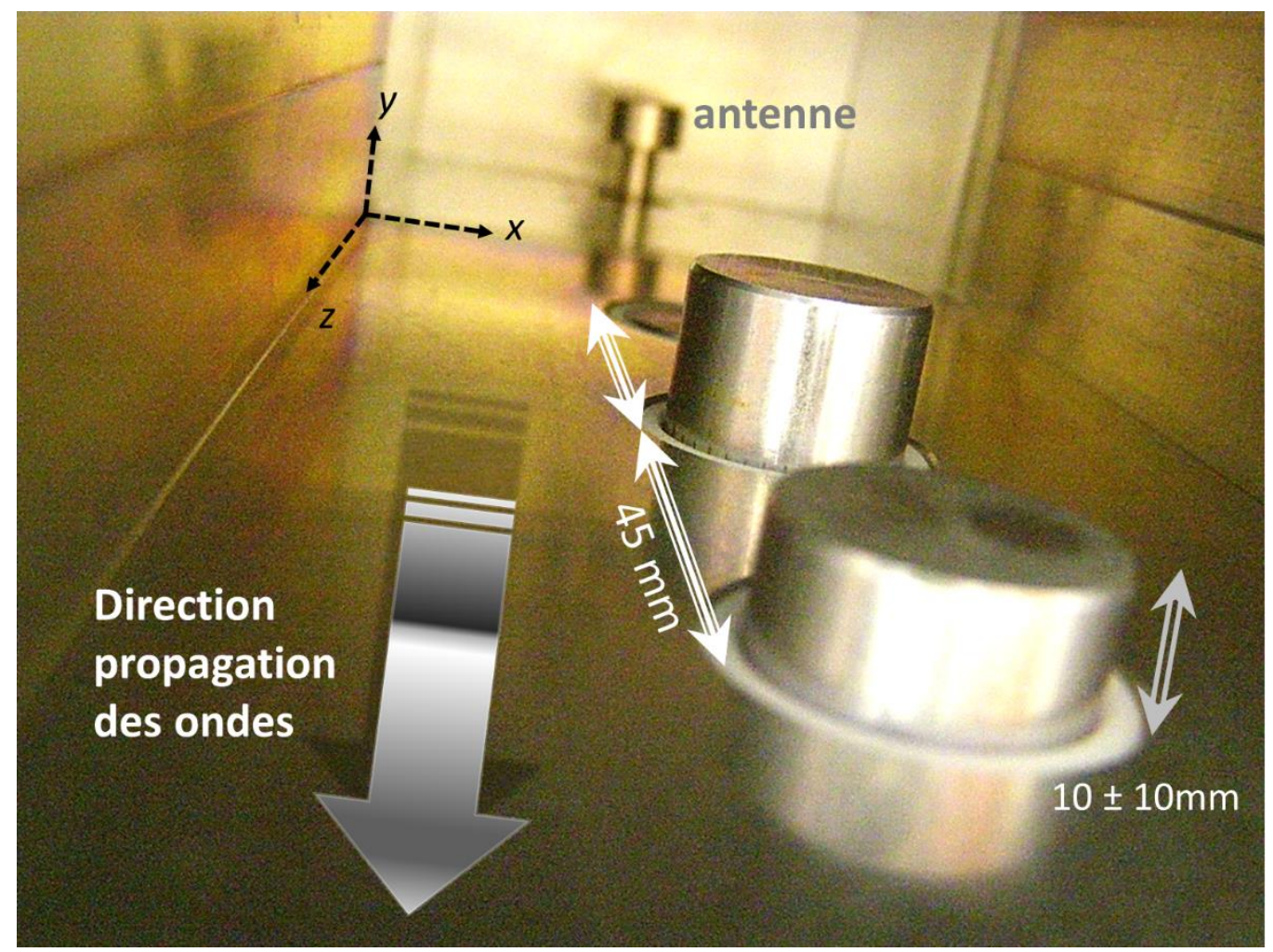

Figure 4.4. Exemple de représentation intérieure d'un adaptateur d'impédance à 3 vis plongeantes (« 3 stub tuner ») dans un guide d'ondes TE10 en aluminium

Le piston de court-circuit (figure 4.1) fait office de plaque métallique et peut se déplacer suivant l'axe de propagation des ondes par mouvements de translation suivant l'axe de propagation. En modifiant la position du piston à l'extrémité du guide, la taille de la cavité est modifiée et cela agit sur le taux d'ondes stationnaires et donc le facteur de réflexion dans la cavité.

Les éléments d'adaptation d'impédance permettent quant à eux d'aider à minimiser la puissance réfléchie par le produit vers le générateur micro-ondes. De ce fait, l'amplitude du champ électrique dans la cavité résonnante peut devenir très importante par rapport à l'amplitude du champ directement en sortie de générateur. Ainsi, avec de bons réglages des éléments d'adaptation (piston + vis), la puissance réfléchie "vue" par le générateur peut tendre vers zéro.

Dans le cas où le piston de court-circuit ne permet pas à lui seul de faire baisser suffisamment la puissance réfléchie, une solution consiste à abaisser la première vis plongeante (la plus proche du produit) pour essayer de minimiser le facteur de réflexion des ondes. Si le signal réfléchi reste faiblement atténué, la seconde vis peut être abaissée à son tour. Il est cependant recommandé de ne pas abaisser les trois vis en même temps dans le guide d'ondes sous peine de générer trop d'échauffement des matériaux.

Les deuxièmes éléments d'adaptation d'impédance rencontrés sont les iris de couplage qui sont en quelque sorte des plaques métalliques positionnées en amont de l'applicateur contenant l'échantillon à traiter. Suivant les applications, une ou plusieurs plaques peuvent être utilisées. Les iris de couplage intégrés dans une cavité résonnante sont souvent considérés comme moins dissipateurs de chaleur par rapport aux vis plongeantes mais leur rôle reste similaire (Charmond, 2009). 
Dans le cas où les propriétés diélectriques du produit à traiter varient beaucoup en fonction de la température (ex: eau distillée dans la gamme $20^{\circ} \mathrm{C}$ à $80^{\circ} \mathrm{C}$ ), il est souvent difficile de minimiser la puissance réfléchie à tout instant au cours de l'opération de chauffage micro-ondes. Dans ce cas particulier, le simple réglage des vis plongeantes ne suffit pas car il faudrait dans certains cas régler en continu la position des vis et du piston afin de réduire le facteur de réflexion au cours de l'opération. Cela est malgré tout possible avec des vis plongeantes motorisées et asservies au générateur microondes. Pour certains générateurs micro-ondes à état solide, une option consiste aussi à faire varier la fréquence des micro-ondes dans un gamme restreinte $(2,4$ à $2,5 \mathrm{GHz}$ par exemple) afin de minimiser la puissance réfléchie en temps réel («frequency shifting »). Cette option de «tuning » automatique est particulièrement intéressante dans le cas où l'on souhaite maximiser à chaque instant le rendement énergétique du procédé. L'inconvénient majeur de cette approche est que la fréquence n'est plus considérée comme une constante (à $2,45 \mathrm{GHz}$ par exemple). Dans un objectif de modélisation de la dynamique du procédé micro-ondes, il est alors nécessaire de prendre en compte les variations fréquentielles lors du traitement micro-ondes afin de quantifier l'impact sur le profil de température obtenu (Resurreccion Jr, Luan et al, 2015).

Connaissant la valeur de la longueur d'onde guidée à vide $(0,173 \mathrm{~m}$ à la fréquence de $2,45 \mathrm{GHz})$, on peut en déduire la taille de la cavité résonnante optimale permettant d'obtenir une propagation en ondes stationnaires dans le guide d'ondes. Dans ce cas, la propagation devient un mode $\mathrm{TE}_{10 p}$, avec $p$ représentant le nombre de demi-sinusoïdes dans le sens de propagation des ondes (axe z). Une cavité résonnante est donc formée entre l'entrée du guide d'ondes et le piston de court-circuit.

Compte tenu de la distance entre l'élément d'adaptation et le piston de court-circuit, il est possible de définir des longueurs minimum et maximum pour que la condition de résonnance soit respectée dans la cavité. Par exemple, prenons le cas d'un dispositif expérimental où la distance entre l'élément d'adaptation le plus proche du produit et le piston de court-circuit peut varier de $384 \mathrm{~mm}$ à $534 \mathrm{~mm}$. La cavité résonnante aura donc une longueur comprise entre ces deux bornes minimum et maximum (soit une amplitude de déplacement du piston de $150 \mathrm{~mm}$ ). Cette amplitude de déplacement permet de translater l'onde stationnaire afin de déplacer les extremum de champs électriques et magnétiques tout au long de l'axe de propagation des ondes.

A vide, à la fréquence de $2450 \mathrm{MHz}$, compte tenu de la demi-longueur d'onde guidée dans l'air $\lambda_{\mathrm{g}} / 2$ $(86,36 \mathrm{~mm})$, plusieurs longueurs de cavités résonnantes $L$ sont envisageables telle que $L_{\min }<p . \lambda_{\mathrm{g}} / 2<$ $L_{\max }$ avec $L_{\min }=384 \mathrm{~mm}$ et $L_{\max }=534 \mathrm{~mm}$ et $p$ un nombre entier (tableau 4.1).

\begin{tabular}{|c|c|c|c|c|c|c|}
\hline Indice $\boldsymbol{p}$ & 2 & 3 & 4 & $\mathbf{5}$ & $\mathbf{6}$ & 7 \\
\hline $\boldsymbol{L}(\mathbf{m m})$ & 172,72 & 259,08 & 345,44 & $\mathbf{4 3 1 , 8}$ & $\mathbf{5 1 8 , 1 6}$ & 604,52 \\
\hline
\end{tabular}

Tableau 4.1. Détermination des longueurs théoriques de cavités résonnantes possibles à vide

D'après les données du tableau 4.1, seuls les modes $\mathrm{TE}_{105}$ et $\mathrm{TE}_{106}$ sont envisageables dans cette cavité résonnante à vide.

Ces valeurs indicatives peuvent constituer une bonne base afin d'estimer les réglages initiaux de la position du piston de court-circuit lors de la mise au point du dispositif.

Malgré tout, quand un échantillon à pertes diélectriques est introduit dans la cavité, ces valeurs de longueurs théoriques pour la cavité résonnante sont modifiées en fonction des propriétés diélectriques de l'échantillon considéré ainsi que de sa géométrie. Il est alors nécessaire de modifier la course du piston ainsi que le réglage des vis plongeantes le cas échéant en fonction de la réponse observée sur la puissance réfléchie. 


\subsection{Mesure de puissance dans les guides d'ondes}

Dès lors qu'un produit est positionné au sein de l'applicateur micro-ondes, une partie de la puissance incidente est absorbée, transmise ou encore réfléchie par le produit traité.

Plusieurs types de technologies peuvent être utilisées pour la mesure de la puissance réfléchie. Ce signal est la plupart du temps mesuré par un isolateur coaxial placé soit à l'intérieur du générateur micro-ondes ou entre le générateur et l'entrée du guide d'ondes au niveau de la transition guide-coaxial (figure 4.1). L'isolateur coaxial consiste en un circulateur couplé à une charge absorbante. Cet isolateur permet en quelque sorte d'isoler le signal réfléchi du circuit micro-ondes principal afin de mesurer la puissance réfléchie. Cette déviation du faisceau est réalisée par l'intermédiaire de ferrites qui dévient le signal réfléchi vers une autre voie pour sa mesure (figure 4.5). Ceci assure par la même occasion la protection du générateur d'une puissance réfléchie trop importante (cas d'une charge non adaptée en impédance ou fortement réfléchissante).

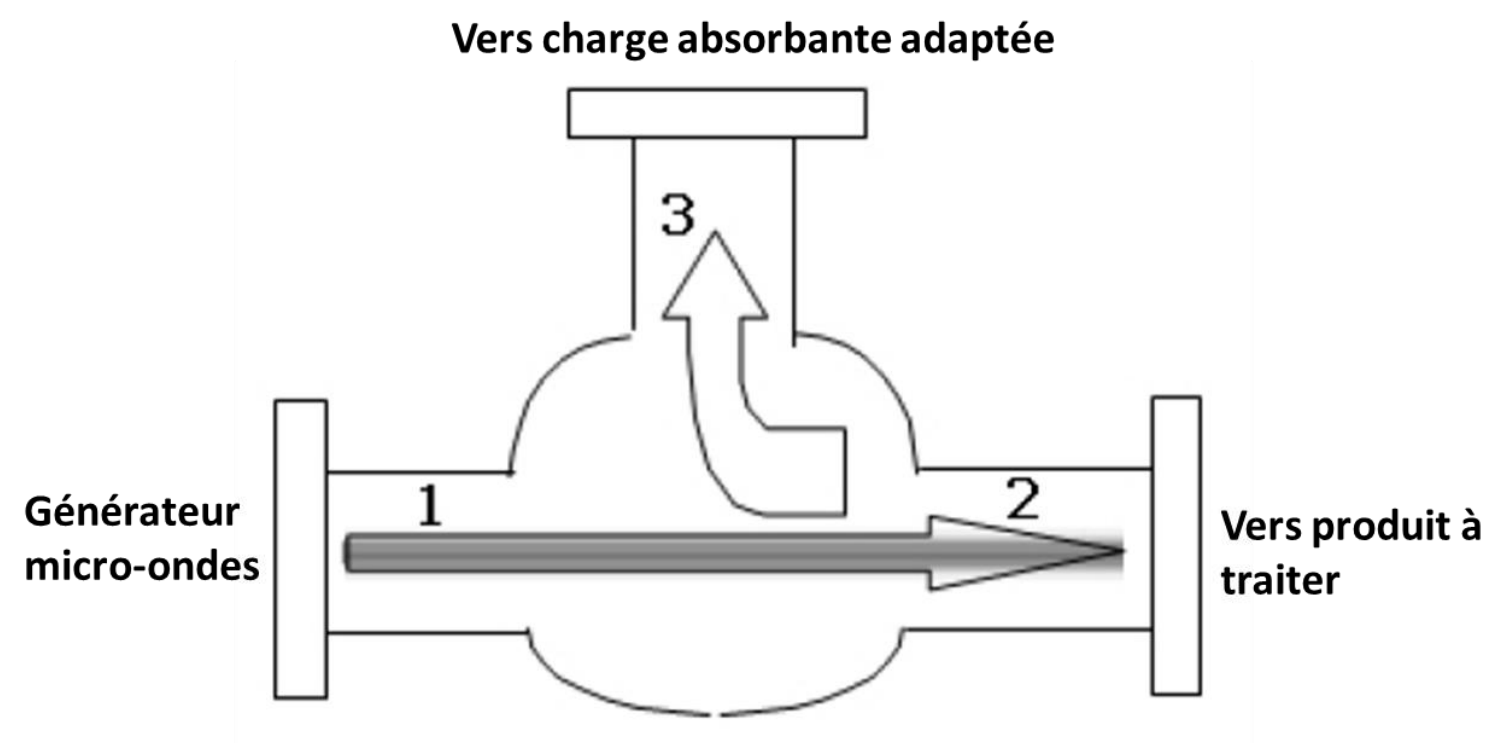

Figure 4.5. Représentation schématique d'un isolateur (d'après Charmond 2009)

Afin de mesurer précisément la puissance transmise au sein d'un guide d'ondes, on peut avoir recourt à un coupleur directionnel. Le principe de fonctionnement de ce type de dispositif est illustré sur la figure 4.6. 


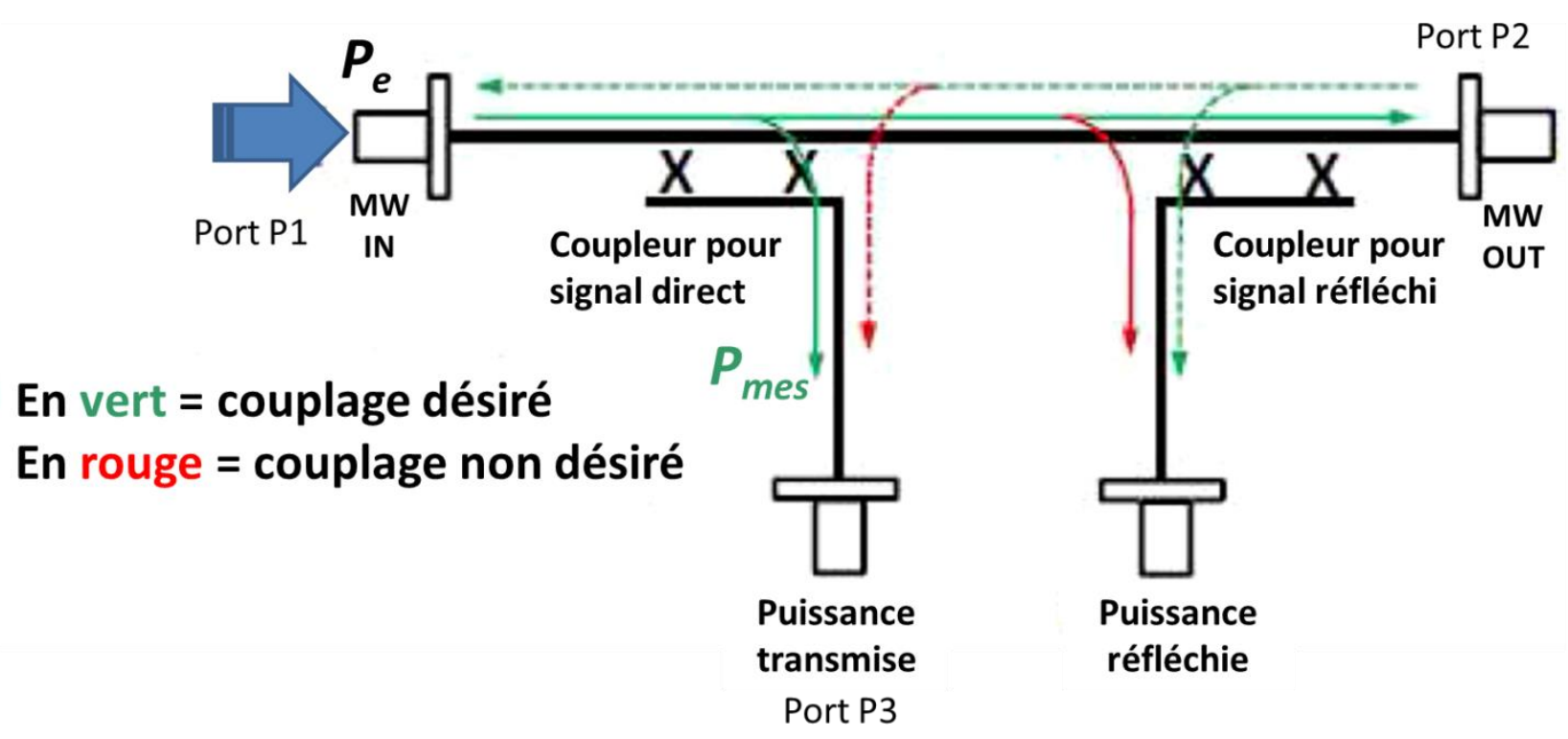

Figure 4.6. Représentation schématique d'un coupleur bidirectionnel

Lorsqu'un courant circule dans la ligne principale du coupleur, une fraction de ce courant se retrouve à l'autre extrémité (port P3) de la ligne de mesure. Le coupleur directionnel permet la mesure du courant direct en ignorant le courant réfléchi et réciproquement.

Le rapport entre la puissance transmise $\left(P_{e}\right)$ sur la ligne principale et la puissance prélevée pour la mesure $\left(P_{\text {mes }}\right)$ est définie par le facteur de couplage $(\mathrm{FC})$. On l'exprime en décibels à l'aide de la relation suivante :

$$
F C=10 . \log \frac{P_{e}}{P_{\text {mes }}}
$$

Par exemple, un coupleur $10 \mathrm{~dB}$ prélève $10 \%$ de la puissance transmise sur la ligne. Si celle-ci est de $100 \mathrm{~W}$, la puissance disponible sur le port de mesure sera de 10 watts, c'est à dire à un niveau -10dB par rapport au niveau sur le port P1 à l'entrée de la ligne principale. La figure 4.4 illustre le principe d'un coupleur bidirectionnel permettant de mesurer à la fois le signal incident et réfléchi dans la ligne de transmission.

\section{Modélisation numérique du chauffage par micro-ondes}

\subsection{Généralités sur les méthodes numériques utilisées}

Du fait de la complexité des phénomènes physiques inhérents à un procédé de chauffage par microondes, les mesures des paramètres du procédé en temps réel sont très difficiles et l'installation de capteurs spécifiques à différents endroits du matériau traité reste encore limité. La simulation numérique du traitement thermique par micro-ondes est donc un outil très intéressant pour évaluer, optimiser et mettre à l'échelle un procédé. En particulier, la modélisation de la distribution spatiale de la température dans l'ensemble du produit est essentielle pour prédire l'efficacité du processus de chauffage dans le temps, assurant ainsi la qualité microbiologique du produit et une bonne efficacité énergétique.

Les équations aux dérivées partielles sont à la base des modèles physiques qui décrivent le processus de chauffage par micro-ondes. Les solutions analytiques de ces équations ne sont pas faciles à obtenir dans les cas réels avec des géométries complexes (Knoerzer et al, 2011). Pour faire face à ces difficultés et à ces aspects, il convient donc de choisir une méthode numérique appropriée. Il existe 
plusieurs techniques numériques pour simuler le chauffage par micro-ondes, parmi lesquelles deux méthodes ont été principalement employées : la méthode « Finite Difference Time Domain » (FDTD) et la méthode des éléments finis (FEM) (Dibben, 2001). Moins fréquemment, la méthode des moments et la méthode de la ligne de transmission ont également été appliquées (Chen et Tang, 2011 ; Knoerzer et al, 2011).

En bref, la méthode FDTD est une procédure par pas de temps qui simule les ondes électromagnétiques continues dans une région spatiale finie jusqu'à ce que le temps de simulation désiré soit atteint ou qu'un modèle de champ électromagnétique stable soit établi (Chen et Tang, 2011). Dans la méthode FDTD, les équations de Maxwell sont résolues alternativement. Le champ électrique au temps $t$ est utilisé pour calculer le champ magnétique au temps $t+1 / 2 \Delta t$, qui à son tour est utilisé pour calculer le champ électrique au temps $t+\Delta t$, et ainsi de suite (Dibben, 2001). Le principal inconvénient de cette méthode est dû à l'utilisation d'une grille régulière. De plus, la méthode FDTD nécessite une mémoire excessivement importante, en particulier dans le cas de géométries complexes ; le domaine doit être suffisamment discrétisé pour résoudre les différentes longueurs d'onde et les caractéristiques géométriques, ce qui entraîne des temps de calcul importants (Choi et al, 2015).

Dans la méthode des éléments finis, la géométrie étudiée est décomposée en une union de petits éléments de géométrie simple, généralement des quadrilatères ou triangles en deux dimensions et des tétraèdres ou hexaèdres en trois dimensions (Dibben, 2001). Fondamentalement, les quatre étapes principales suivantes sont appliquées dans la méthode FEM : division de la région/géométrie de la solution en plusieurs sous-régions ou éléments ; obtention de l'équation aux dérivées partielles dans chaque élément; intégration des solutions de tous les éléments dans la solution pour la géométrie complète et enfin résolution numérique des équations obtenues (Chen et Tang, 2011). Comme la méthode FEM ne nécessite pas de grille régulière, la décomposition de la géométrie est très flexible. De ce fait, la méthode n'a aucune restriction, même avec des géométries arbitraires et irrégulières, et elle peut traiter tout type de conditions aux limites (Choi et al., 2015). Cette méthode est donc utile pour les géométries très complexes (Chen et Tang, 2011). La méthode FEM a été largement utilisée pour modéliser le chauffage par micro-ondes des produits alimentaires en raison de sa flexibilité dans la gestion des géométries irrégulières et des conditions aux limites, des variations des propriétés spatiales et temporelles (Liu et al., 2013 ; Romano et al., 2005).

\subsection{Modélisation de la source de chaleur électromagnétique}

La modélisation est un outil permettant par exemple de prédire la distribution de la température en fonction du temps et l'intensité du champ électrique à l'intérieur du produit ; elle est donc considérée comme un bon moyen d'évaluer l'efficacité du chauffage par micro-ondes. Le modèle de chauffage par pertes diélectriques peut être basé sur deux approches de modélisation de la source de chaleur: l'application de la loi de Lambert et la résolution complète des équations de Maxwell.

Les équations de Maxwell régissent la propagation du champ électromagnétique et l'utilisation de ces équations dépend de la connaissance des propriétés diélectriques (Hamoud-Agha et al., 2013; Oliveira et Franca, 2002). Les équations de Maxwell ont déjà été présentées dans le paragraphe 3.1. L'approche de la loi de Lambert suppose une décroissance exponentielle de la puissance absorbée par micro-ondes en fonction de la profondeur dans l'échantillon (Hamoud-Agha et al., 2013; Vadivambal et Jayas, 2010). Comme évoqué précedemment, il s'agit d'une approximation pour déterminer l'intensité de la puissance micro-ondes en fonction de l'épaisseur du produit (Vilayannur et al., 1998). Contrairement à la méthode classique des équations de Maxwell, l'approche utilisant la loi de Lambert n'exige pas le calcul du champ électromagnétique local dans le produit traité (Curet et al., 2008a).

L'application de la loi de Lambert peut fournir des résultats numériques proches des mesures expérimentales, mais seulement dans des conditions spécifiques. Selon Liu et al (2005) et Curet et al (2008a), les calculs utilisant les équations de Maxwell sont plus rigoureux, tandis que les approches 
fondées sur la loi de Lambert sont moins complexes et conduisent à des temps de calcul plus courts. Le choix de l'approche la plus appropriée varie en fonction de la situation. Dans certains cas, l'application de la loi de Lambert est possible, et dans d'autres, la résolution complète des équations de Maxwell est strictement requise.

En général, la loi de Lambert peut être utilisée pour évaluer le chauffage par micro-ondes pour des échantillons de largeur semi-infinie et suffisamment épais devant la profonfeur de pénétration des ondes (Vadivambal et Jayas, 2010). Lorsque l'épaisseur du produit dans le sens de propagation des micro-ondes n'est pas assez importante, le chauffage doit être calculé à partir de la résolution complète des équations de Maxwell (Cheng et al, 1997). Dans le cas d'un échantillon d'épaisseur faible devant la profondeur de pénétration des micro-ondes, des configurations d'ondes stationnaires peuvent apparaître en raison des composantes de réflexion et de transmission à l'intérieur du matériau, ce qui nécessite la résolution complète du champ (Curet et al, 2008a).

Dans le cas d'un gel d'agar cylindrique, l'approche de la loi de Lambert a donné lieu à une distribution de température moins précise que celle fondée sur les équations de Maxwell, particulièrement au niveau des bords de l'échantillon (Yang et Gunasekaran, 2004). Généralement pour les géométries courbes, l'approche de la loi de Lambert n'est pas appropriée pour prédire l'effet de focalisation par micro-ondes et ainsi, l'ensemble complet des équations de Maxwell doit être considéré (Datta, 2001).

Une autre situation pour laquelle les équations de Maxwell sont nécessaires est le processus de décongélation par micro-ondes. En particulier, la loi de lambert ne prédit pas avec précision la distribution de puissance micro-ondes absorbée en phase congelée à cause des phénomènes de résonance non pris en compte (Curet et al., 2008a).

\subsection{Modélisation du transfert de chaleur sous micro-ondes}

\subsubsection{Equation générale du transfert thermique avec génération de chaleur micro-ondes}

L'équation générale pour le transfert de chaleur dans un milieu solide sans convection peut être décrite comme suit (Knoerzer et al., 2011) :

$$
\rho C_{p} \frac{\partial T}{\partial t}-\nabla \cdot(k \cdot \nabla T)=-\nabla \cdot q_{R}+Q_{g e n}
$$

où $\rho$ est la densité, $C_{p}$ est la capacité calorifique massique et $k$ est la conductivité thermique du matériau. $q_{R}$ représente la densité du flux liée au rayonnement et $Q_{g e n}$ est la source de chaleur volumique. Dans le cas d'un chauffage par micro-ondes, le membre gauche de l'équation précédente décrit la conduction thermique et les termes de droite représentent le transfert de chaleur par rayonnement et la source de chaleur par pertes diélectriques (Knoerzer et al., 2011).

Dans le cas du chauffage par micro-ondes, la génération de chaleur (ou densité de puissance volumique) dépend des valeurs des propriétés diélectriques du produit et du carré du champ électrique local (Curet et al, 2008b).

$$
Q_{g e n}=\frac{1}{2} \omega \varepsilon_{0} \varepsilon_{r}^{\prime \prime}|E|^{2}
$$

Il est à noter que dans l'équation présentée ci-dessus, le terme relatif au transfert de masse (loi de Fick) a été négligé. Cependant, lorsque le problème étudié présente un transfert de chaleur avec prise en compte du transfert de masse (ex. changement de phase de l'eau ; diffusion ou convection), un terme supplémentaire doit être inclus et l'équation devient (Knoerzer et al., 2005) : 


$$
\rho C_{p} \frac{\partial T}{\partial t}-\nabla \cdot(k \cdot \nabla T)=-\nabla \cdot q_{R}+Q_{g e n}-\sum_{i} h_{i} I_{i}
$$

où $I_{i}$ est la source ou puit associé à l'enthalpie massique $h_{\mathrm{i}}$ de la phase i. Évidemment, dans d'autres cas spécifiques, comme le processus de séchage et l'étude des matériaux poreux, certaines modifications dans ces équations peuvent être nécessaires.

En complément, si l'on considère un chauffage par micro-ondes d'un liquide à pertes diélectriques, en incluant le terme de convection thermique lié à l'écoulement et en supposant qu'il n'y a pas de transfert de masse, l'équation générale du transfert thermique s'exprime comme suit :

$$
\rho C_{p} \frac{\partial T}{\partial t}+\rho C_{p} \vec{U} \cdot \nabla T-\nabla \cdot(k \cdot \nabla T)=Q_{g e n}
$$

où $\vec{U}$ représente le champs de vitesse dans le fluide.

\subsubsection{Modélisation du chauffage par micro-ondes de produits liquides}

La simulation numérique du chauffage micro-ondes de produits liquides est complexe et exigeante sur le plan des ressources calcul, car elle implique l'interaction de champs électromagnétiques, de transferts thermiques et de phénomènes liés à la mécanique des fluides. Dans le cas du chauffage par micro-ondes de liquides, une source de chaleur volumique non uniforme provoque des modifications de température qui entraînent des différences de densité du fluide et donc un mouvement du fluide par convection naturelle. En complément, le mouvement du fluide modifie la distribution de la température, affectant les propriétés diélectriques du produit et donc la distribution du champ électromagnétique dans le volume traité. Par conséquent, en raison de la complexité des distributions de puissance et de la présence d'un flux de chaleur induit par la convection, cela entraîne des couplages forts entre les différentes physiques. La modélisation du transfert de chaleur de produits liquides sous micro-ondes peut ainsi devenir relativement complexe (Chatterjee et al., 2007 ; Zhang et al., 2000).

Les premiers travaux concernant la modélisation du chauffage par micro-ondes ont traité de configurations en cavités avec des matériaux solides. De nos jours, grâce aux progrès informatiques et aux améliorations des logiciels, de plus en plus d'études numériques sur le chauffage par micro-ondes de produit liquides sont remarquées (Chatterjee et al., 2007 ; Chen et al., 2016 ; Choi et al., 2015 ; Curet et al., 2015 ; Klinbun et Rattanadecho, 2016 ; Ratanadecho et al, 2002 ; Salvi et al, 2011 ; Tuta et Palazoglu, 2017 ; Yeong et al, 2017 ; Zhang et al, 2000 ; Zhu et al, 2007).

Zhang et ses collaborateurs (2000) ont étudié le chauffage par micro-ondes de deux liquides en statique : l'eau distillée et l'huile de maïs. Les auteurs ont conclu que la convection joue un rôle significatif sur la distribution de la température lors du chauffage micro-ondes de produits liquides.

Salvi et ses collaborateurs (2011) ont étudié un procédé de chauffage par micro-ondes à flux continu utilisant une solution de carboxyméthylcellulose (fluide non newtonien) et de l'eau (fluide newtonien). Un modèle numérique a été développé pour simuler les profils de température via un couplage itératif entre le champ électromagnétique, l'écoulement des fluides et le transfert de chaleur pour les deux fluides. Une étude similaire a été menée par Tuta et Palazoglu (2017). Leurs travaux ont consisté à étudier le chauffage micro-ondes de l'eau distillée et de solutions de carboxyméthylcellulose dans un fluide en écoulement continu. Les résultats numériques et expérimentaux ont montré que le chauffage de l'eau était plus uniforme que celui des solutions de carboxyméthylcellulose à tous les débits testés.

Les équations gouvernant l'écoulement des fluides sont basées sur la conservation de la masse (équation de continuité), de la quantité de mouvement (deuxième loi de Newton) et de l'énergie 
(première loi de la thermodynamique), et peuvent également être basées sur des équations d'état reliant les propriétés physiques des matériaux aux conditions du procédé, comme l'approximation de Boussinesq (Norton et Sun, 2006 ; Scott et Richardson, 1997).

L'équation de continuité est basée sur le principe de la conservation de masse. Elle peut être exprimée sous la forme d'une équation différentielle de la forme :

$$
\frac{\partial \rho}{\partial t}+\nabla \cdot(\rho \vec{U})=0
$$

où $\vec{U}$ est le vecteur vitesse et $\rho$ la masse volumique du fluide.

L'équation de conservation de la quantité de mouvement est liée au taux d'accumulation de la quantité de mouvement par convection, au taux d'accumulation de la quantité de mouvement par transfert moléculaire et à la somme des forces agissantes sur le système. L'équation de la quantité de mouvement est également connue sous le nom d'équation de Navier-Stokes. Cependant, habituellement, le terme Navier-Stokes est utilisé pour inclure non seulement la quantité de mouvement, mais aussi l'équation de l'énergie et les équations de continuité. Pour un fluide newtonien, l'équation de conservation de la quantité de mouvement peut être exprimée comme suit :

$$
\rho \frac{\partial \vec{U}}{\partial t}=-\nabla \cdot(\rho \vec{U} \times \vec{U})-\nabla p+\nabla \cdot \sigma_{N S}+\overrightarrow{\mathrm{B}}
$$

où $\sigma_{N S}$ est le tenseur de forces visqueuses et $\vec{B}$ est la force agissante dépendant du type d'écoulement liquide. Dans le cas de l'approximation de Boussinesq et là où la seule force exercée est le poids, la force devient : $\vec{B}=\rho \vec{g}$ (Scott et Richardson, 1997), avec $g$ l'accélération gravitaire.

L'équation de la quantité de mouvement est couplée à l'équation de la chaleur via le terme de convection. Ainsi, une autre forme de l'équation générale du transfert de chaleur présente les termes relatifs au taux d'accumulation par convection et conduction et au travail des forces effectué par l'environnement. Elle peut s'exprimer comme suit :

$$
\frac{\partial \rho h_{T}}{\partial t}=-\nabla \cdot\left(\rho \vec{U} h_{T}\right)+\nabla \cdot(k \nabla T)+\frac{\partial p}{\partial t}
$$

où $h_{T}$ est l'enthalpie totale du système considéré, $k$ est la conductivité thermique et $p$ est la pression du fluide. Souvent, le terme de pression du côté droit de l'équation est négligé. Dans le cas d'un modèle de simulation couplant le chauffage hyperfréquence et le débit du fluide, l'équation à résoudre est alors similaire à celle présentée dans la section 5.3.1.

$$
\rho C_{p} \frac{\partial T}{\partial t}+\rho C_{p} \vec{U} \cdot \nabla T-\nabla \cdot(k \cdot \nabla T)=Q_{g e n}
$$

Une équation d'état couramment utilisée est basée sur l'approximation de Boussinesq, dans laquelle toutes les propriétés du fluide sont supposées indépendantes de la température, excepté la densité.

$$
\rho=\rho_{\text {ref }}\left[1-\beta\left(T-T_{\text {ref }}\right)\right]
$$

où $\beta$ est le coefficient de dilatation thermique, $\rho_{\text {ref }}$ et $T_{r e f}$ sont respectivement la densité et la température de référence. Cette approximation permet de générer un mouvement du fluide par convection du fait de la dépendance en température de la densité.

Dans les logiciels de simulation numérique, la solution numérique des équations de quantité de mouvement est fonction du type de fluide considéré : incompressible, compressible ou faiblement 
compressible. Il est bien connu que les liquides sont beaucoup moins compressibles que les gaz. Habituellement, pour les liquides sous certaines conditions, le fluide peut être considéré comme incompressible. Cela signifie que la densité est supposée constante et que l'équation de continuité peut être simplifiée telle que :

$$
\nabla \cdot(\vec{U})=0
$$

Généralement, dans ces situations, l'approximation de Boussinesq peut également être prise en compte dans l'équation de la conservation de la quantité de mouvement. L'approximation de Boussinesq indique alors que la variation de densité n'est importante que dans le terme de gravitation, et peut être négligée dans le reste de l'équation de la conservation de la quantité de mouvement, de telle sorte que :

$$
\rho_{\text {ref }} \frac{\partial \vec{U}}{\partial t}+\rho_{\text {ref }}(\vec{U} \cdot \nabla) \vec{U}=\nabla \cdot\left[-p I+\mu\left(\nabla \vec{U}+(\nabla \vec{U})^{T}\right)-\frac{2}{3} \mu(\nabla \cdot \vec{U}) I\right]-\rho \overrightarrow{\mathrm{g}}
$$

Néanmoins, dans certains cas, cette approximation ne peut être faite et le couplage entre la vitesse, la pression et la température est nécessaire (fluide compressible). Dans d'autres cas, on peut supposer un scénario intermédiaire. Le fluide peut être considéré comme faiblement compressible et la densité dépend alors de la température mais pas de la pression. Dans cette dernière situation, l'équation de la quantité de mouvement est la suivante :

$$
\rho \frac{\partial \vec{U}}{\partial t}+\rho(\vec{U} \cdot \nabla) \vec{U}=\nabla \cdot\left[-p I+\mu\left(\nabla \vec{U}+(\nabla \vec{U})^{T}\right)-\frac{2}{3} \mu(\nabla \cdot \vec{U}) I\right]-\rho \overrightarrow{\mathrm{g}}
$$

où $I$ est la matrice identité et $\mu$ est la viscosité dynamique du fluide.

Globalement, au vu des équations présentées, il convient de noter l'importance des propriétés thermophysiques et diélectriques pour prédire au mieux la cartographie thermique dans le fluide. En effet, pour simuler et prédire avec précision les effets thermiques des processus de chauffage par micro-ondes, il est nécessaire de considérer des équations corrélant les propriétés thermophysiques et diélectriques des matériaux alimentaires aux variations de température.

Comme nous l'avons vu dans les sections précédentes, ces propriétés jouent un rôle important dans les équations. Par conséquent, l'utilisation de valeurs approximatives en considérant ces propriétés comme indépendantes de la température donnera très probablement des résultats moins précis. De plus, il est nécessaire de considérer l'importance du couplage électromagnétique avec le transfert de chaleur et l'écoulement du fluide, puisque chacun de ces phénomènes sont interdépendants.

\section{Les exemples de traitements thermiques micro-ondes dans l'industrie alimentaire}

\subsection{Généralités}

Comme illustré sur la figure 6.1, le chauffage par micro-ondes est habituellement utilisé pour des opération unitaires telles que la pasteurisation, la stérilisation, le blanchiment, le séchage, la concentration, la décongélation, la cuisson, la torréfaction, l'extraction, ou encore une assistance à la congélation (Ballard et al., 2010 ; Bozkir et Baysal, 2017a ; Cañumir et al, 2002 ; Chahbani et al, 2018 ; Dahmoune et al, 2015 ; Güneş and Bayindirli, 1993 ; Kim et al, 2018 ; Luter et al, 1982 ; Marić et al, 2018 ; Monteiro et al, 2018 ; Pérez-Grijalva et al, 2018 ; Siguemoto et al, 2017 ; Silva et al, 2006 ; Simić et al, 2016 ; Taher et Farid, 2001 ; Xanthakis et al, 2014). D'autres domaines d'application concernent les procédés de torréfaction, pyrolyse de la biomasse et la transestérification des lipides (Antunes et al., 2018 ; Chee Loong et Idris, 2014 ; Yin, 2012). 
Parmi les applications industrielles et commerciales de la technologie de chauffage par micro-ondes dédié à la transformation des aliments, on retrouve : la précuisson du bacon, la cuisson des saucisses et de la viande, le séchage des croustilles de pommes de terre, des légumes, la cuisson du pain, la décongélation des produits congelés, le blanchiment des légumes, le séchage par micro-ondes des jus de fruits sous vide, le chauffage, la pasteurisation des pâtes et des plats préparés (Schiffmann, 2001 ; Vadivambal and Jayas, 2010).

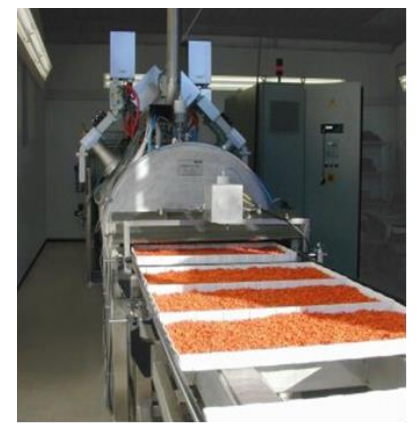

Séchage

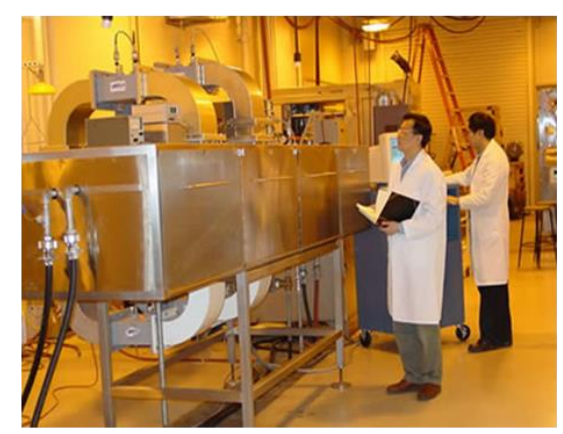

Pasteurisation



Cuisson



Tempérage

Figure 6.1. Les récents développements d'utilisation des micro-ondes en industrie alimentaire : opérations unitaires majeures

Dans l'industrie agroalimentaire, la technologie micro-ondes est aussi largement utilisée pour le tempérage de la viande et de la volaille, comme procédé de prétraitement. Au cours du processus de tempérage, le produit congelé $\left(<0{ }^{\circ} \mathrm{C}\right)$ est réchauffé par micro-ondes, de sorte que le produit après tempérage deviennent plus malléable $\left(-5\right.$ à $\left.-2{ }^{\circ} \mathrm{C}\right)$, ce qui permet de le trancher, de le couper en dés et de le broyer (Schiffmann, 2001). Le tempérage conventionnel à l'air entraîne un gradient de température élevé dans le produit (de la surface vers le cœur) et peut conduire à des temps de traitement assez longs en fonction du calibre du produit. A l'inverse, le tempérage par micro-ondes est relativement rapide avec par exemple 5 à 10 minutes de traitement pour 15 à $40 \mathrm{~kg}$ de viande congelée (Schiffmann, 2001).

\subsection{Les avantages d'un traitement par micro-ondes par rapport à un procédé de chauffage conventionnel}

Le chauffage conventionnel repose sur le processus de conduction et de convection pour transférer la chaleur provenant d'une source extérieur (vapeur, eau chaude,...). Comme la conductivité thermique associée à la diffusivité thermique des produits alimentaires est relativement faible, ce processus necessite des temps de traitement relativement longs (Vadivambal et Jayas, 2010). Au cours d'un procédé de chauffage conventionnel, la chaleur se diffuse de la surface extérieure vers le cœur du produit. A l'inverse, le chauffage par micro-ondes a le potentiel de générer de la chaleur dans l'ensemble du produit en raison de la génération de chaleur volumique, ce qui entraîne une réduction significative du temps de procédé. La chaleur est générée à l'intérieur du volume et le gradient de température peut se retrouver inversé par rapport au procédé de chauffage conventionnel. Le problème 
des dommages de la surface du produit dus à une surchauffe par le procédé conventionnel est ainsi évité.

En raison de vitesses de chauffage plus rapides et de temps de traitement plus courts, le chauffage par micro-ondes préserve généralement mieux les composés thermolabiles, maintient l'activité antioxydante, et réduit les changements dans les attributs sensoriels des produits, notamment la couleur, la texture et l'arôme (Güneş et Bayindirli, 1993 ; Guo et al, 2017 ; Marszałek et al, 2015 ; Pérez-Grijalva et al., 2018). Par exemple, Güneş et Bayindirli (1993) ont signalé une rétention plus élevée d'acide ascorbique lorsque les légumes sont traités par micro-ondes comparativement au blanchiment à l'eau chaude. Cano et ses collaborateurs (1997) ont observé que les traitements par micro-ondes entraînaient de légers changements de couleur et de faibles modifications de la composition quantitative et qualitative des caroténoïdes dans la papaye et des anthocyanines dans le fraisier.

Dans le cas particulier du séchage, l'utilisation des micro-ondes peut entraîner une rétractation plus faible du produit, des vitesses de séchage et de réhydratation plus élevés et une consommation d'énergie moindre que par les méthodes de séchage traditionnelles (Guo et al., 2017 ; Schiffmann, 2001). Par exemple, Monteiro et ses collaborateurs (2018) ont constaté que le procédé novateur de séchage micro-ondes sous vide produisait des tranches de citrouille à structure poreuse en un temps de traitement très court. Les temps de séchage s'est révélé au moins douze fois plus courts par rapport à la méthode traditionnelle de séchage à l'air.

Dans le contexte de l'application de la technologie micro-ondes dédié à l'inactivation enzymatique et microbienne dans les matrices alimentaires, certains auteurs ont affirmé l'existence d'effets non thermiques. De cette façon, ces effets seraient complémentaires aux effets thermiques et le traitement par micro-ondes permettrait une plus grande efficacité de l'inactivation. Néanmoins, ce sujet est encore très controversé dans la littérature (voir paragraphe 6.4).

Un autre avantage attribué aux systèmes de chauffage par micro-ondes est le fait de ne pas avoir recours à un fluide caloporteur intermédiaire pour chauffer le produit. De plus, l'utilisation de procédés micro-ondes peut également réduire les temps de nettoyage des installations et ainsi le volume des fluides de nettoyage utilisés.

Par conséquent, le chauffage par micro-ondes est une technologie prometteuse en raison de sa faible consommation d'énergie et d'eau, de son efficacité énergétique accrue, de son temps de chauffage rapide, d'un environnement de travail plus propre, et la possibilité de produire des aliments sains de haute qualité nutritionnelle et sensorielle.

\subsection{Limitations du processus de chauffage par micro-ondes}

De nombreux facteurs physiques ont une influence sur le chauffage par micro-ondes et la distribution de la chaleur volumique au sein du produit traité. Parmi elles, les propriétés diélectriques sont les principales caractéristiques influentes qui peuvent varier, entre autres, avec la densité des produits alimentaires, leur composition physico-chimique, la teneur en eau, la fréquence des microondes et la température (Chandrasekaran et al, 2013 ; Kubo et al, 2018a ; Salvi et al., 2009). Dans une étude expérimentale et numérique, Cheng et ses collaborateurs (1997) ont évalué la distribution de la température pendant le chauffage par micro-ondes d'échantillons cylindriques contenant divers produits modèles (gel d'agar, pâte de bentonite, purée de pommes de terre et pâte synthétique) ayant des propriétés diélectriques différentes. La répartition de la température est modifiée selon les propriétés diélectriques des produits et les points chauds se déplacent de l'environnement externe du cylindre vers le centre à mesure que la profondeur de pénétration des micro-ondes augmente. Ainsi, ces résultats confirment l'influence des propriétés diélectriques des produits sur la distribution de température lors du chauffage par micro-ondes. 
De plus, outre les propriétés diélectriques, la distribution de température dans le produit dépend également de la conception de la cavité micro-ondes (taille et géométrie, distribution du champ électrique, fréquence utilisée et puissance de l'onde électromagnétique incidente). Ainsi, les paramètres géométriques tels que le placement du produit dans la cavité, la forme et la taille associés aux propriétés thermophysiques (conductivité thermique, chaleur spécifique, viscosité) et diélectriques doivent être pris en compte pour optimiser le traitement micro-ondes des produits alimentaires (Anantheswaran and Liu, 1994 ; Salvi et al., 2009 ; Vadivambal et Jayas, 2010).

Par exemple, Vilayannur et ses collaborateurs (1998) ont étudié l'effet de la taille et de la forme de différents échantillons de pommes de terre (briques, cylindre et prisme hexagonal) suivant différents volumes $\left(75,90\right.$, et $\left.105 \mathrm{~cm}^{3}\right)$ sur les performances du chauffage par micro-ondes. Au moyen d'un modèle basé sur des éléments finis, les auteurs ont effectué différentes simulations selon la forme et la taille des échantillons. Pour le cylindre, le point chaud était localisé au centre du produit. Cependant, dans le produit hexagonal en forme de prisme, le centre était froid et les points chauds se trouvaient le long de la frontière. Dans les produits en forme de brique, les points chauds se trouvaient aux coins et les points froids au centre géométrique. La plus faible non-uniformité de température a été observée dans le cas des produits en forme de prisme hexagonal, tandis que la plus forte non-uniformité a été observée dans les produits cylindriques de faible volume $\left(75 \mathrm{~cm}^{3}\right)$.

Un chauffage uniforme et efficace dépend de la bonne intégration de tous ces paramètres en même temps. En raison du grand nombre de facteurs qui influencent le processus, il est difficile d'obtenir une distribution de température uniforme prédéfinie. Même si le chauffage diélectrique est généralement considéré comme plus uniforme que de nombreuses méthodes de chauffage conventionnelles par conduction, la distribution non uniforme de la température est l'un des principaux inconvénients associés au chauffage par micro-ondes (Chandrasekaran et al., 2013 ; Chen et al., 2014 ; Vadivambal et Jayas, 2010).

Le chauffage non uniforme est particulièrement problématique pour les aliments solides et les aliments congelés en cours de décongélation (puisque le passage de la glace à l'eau liquide entraîne une augmentation considérable du facteur de pertes et donc un potentiel emballement thermique du produit). Dans les aliments liquides, le problème se pose également, mais il peut être moins grave car les différences de température peuvent être atténuées par le mélange et le mouvement convectif du liquide. Cependant, la convection naturelle provoquée par le chauffage d'un liquide dépend des propriétés thermophysiques du produit. Par exemple, une augmentation de la viscosité du liquide entraîne une diminution des mouvement convectifs et peu conduire à une distribution hétérogène de la température (Prosetya et Datta, 1991).

La non-uniformité de température est un facteur limitant qui freine l'application de la technologie de chauffage par micro-ondes dans l'industrie agroalimentaire. La distribution non uniforme de la température affecte non seulement l'efficacité énergétique du traitement micro-ondes (en raison de la surchauffe locale provoquée lors d'un emballement thermique), mais soulève également la question de la qualité et de la sécurité alimentaire lorsque les enzymes et micro-organismes indésirables ne sont pas inactivés dans les points froids du produit. Comme les effets thermiques demeurent le principal mécanisme létal connu dans le traitement par micro-ondes, un chauffage non uniforme entraine probablement une inactivation non uniforme. Dans le cas de produits solides séchés par micro-ondes, une distribution hétérogène de la température peut aussi ientraîner une distribution non uniforme de la teneur en eau dans le produit, ce qui entraîne le séchage sélectif de certaines parties et affecte la texture finale du produit (Vilayannur et al., 1998).

Certaines propositions visant à améliorer l'uniformité du chauffage micro-ondes ont été signalées. Certains auteurs ont proposé l'utilisation de brasseurs d'ondes et de plateaux tournants pour les cavités micro-ondes multimodes (Cinquanta et al., 2010 ; Cuccurullo et al., 2017 ; Kurniawan et al., 2015 ; Ye et al., 2017), l'application de micro-ondes pulsées (Datta, 2001 ; Gunasekaran et Yang, 2007), le 
contrôle de la géométrie des échantillons (Vadivambal et Jayas, 2010 ; Vilayannur et al, 1998), le traitement à basse fréquence (896 ou $915 \mathrm{MHz}$ au lieu de $2450 \mathrm{MHz}$ pour augmenter la profondeur de pénétration), et le traitement combiné micro-ondes avec infrarouge ou air chaud, notamment pour les procédés de séchage (Guo et al., 2017 ; Ren et Chen, 1998). Il a également été suggéré d'utiliser des systèmes continus ou semi-continus utilisant des bandes transporteuses ou des écoulements tubulaires au lieu de systèmes discontinus (Atuonwu et Tassou, 2018). Toutefois, il convient de rappeler que ces propositions visant à réduire la non-uniformité de chauffage sont basées sur des conditions opératoires spécifiques inhérentes à chaque produit et procédé, et ne peuvent donc être généralisées à aucune autre application.

\subsection{Présence d'éventuels effets non-thermiques}

L'existence possible d'effets non thermiques des micro-ondes sur les matrices alimentaires est certainement un sujet très controversé dans la littérature. Ceci est principalement lié à la complexité du phénomène de chauffage par micro-ondes, qui dépend de plusieurs facteurs, comme décrit précédemment. Selon Anantheswaran et Ramaswamy (2001) et Ramaswamy et al (2002), quatre types d'effets peuvent exister au cours d'un traitement par micro-ondes: les effets thermiques, non thermiques (ou athermiques) qu'ils soient renforcés ou spécifiques.

Les effets thermiques sont ceux qui s'expliquent par l'élévation de la température au sein d'une matrice. Globalement, ces effets sont bien acceptés par la communauté scientifique et c'est la chaleur générée qui est responsable de l'inactivation des microorganismes et des enzymes et de la dégradation des composés de la même manière que dans les procédés de chauffage conventionnels.

Les effets non thermiques des micro-ondes sont des effets qui ne peuvent être expliqués par l'augmentation macroscopique de la température, l'historique temps-température ou les gradients de température (Ramaswamy et al., 2002). Les cas où le chauffage par micro-ondes produit des effets particuliers qui ne peuvent être obtenus par d'autres méthodes de chauffage sont considérés comme des effets micro-ondes spécifiques (Shamis et al., 2012). La définition des effets thermiques renforcés est basé sur les réactions organiques en considérant que le chauffage par micro-ondes ne modifie pas fondamentalement les mécanismes réactionnels, mais facilite la réaction en réduisant le temps global (Anantheswaran et Ramaswamy, 2001 ; Ramaswamy et al., 2002).

D'un point de vue pratique, ces termes sont assez confus et la distinction entre chacun d'eux est difficile d'un point de vue expérimental. Par conséquent, la plupart des études de la littérature désignent les effets non thermiques comme tous les phénomènes observés (macroscopiques ou microscopique) à l'exception des effets thermiques. Un aperçu des études controversées portant sur les effets non thermiques des micro-ondes est présenté ci-dessous.

Des recherches approfondies ont été menées pour déterminer l'existence d'effets non thermiques et pour mesurer l'ampleur du phénomène le cas échéant. De tels effets modifieraient le comportement chimique, biochimique ou physique de certains systèmes en raison de l'exposition aux micro-ondes et de l'interaction du champ électrique avec des molécules spécifiques dans le milieu réactionnel (Huang et al., 2009). Plusieurs études ont été réalisées à l'aide de différentes procédures et conceptions expérimentales pour évaluer et éventuellement distinguer les effets thermiques et non thermiques.

Shazman et ses collaborateurs (2007) ont évalué différents systèmes chimiques, biologiques et physiques dans un dispositif à forte densité de puissance micro-ondes $(2,45 \mathrm{GHz}$, jusqu'à $1000 \mathrm{~W} / \mathrm{kg}$, avec un rayonnement continu jusqu'à 48h). Les systèmes qui ont été testés incluaient plusieurs transformations : la réaction de Maillard, la dénaturation des protéines et la solubilité des polymères, la mutagenèse des bactéries, le phénomène de mutarotation du D-glucose, et la solubilité à saturation du chlorure de sodium. Aucun effet non thermique significatif n'a été observé dans aucun des systèmes étudié. 
En ce qui concerne l'inactivation microbienne, certains chercheurs ont trouvé des preuves qui renforcent l'existence d'effets non thermiques. Dreyfuss et Chipley (1980) ont étudié les effets du rayonnement micro-ondes à $2,45 \mathrm{GHz}$ sur l'activité cellulaire métabolique de Staphylococcus aureus à une température maximale de $46{ }^{\circ} \mathrm{C}$. Les résultats ont été comparés au chauffage conventionnel et des différences considérables ont été constatées. Les auteurs ont donc signalé l'existence possible d'effets non thermiques. Cependant, comme la température n'a pas été très bien contrôlée pendant le chauffage, l'exactitude des résultats rapportés peut être questionnée.

Zhang et al. (2012) ont conçu une cavité coaxiale hyperfréquence dans laquelle l'échantillon a été soumis à un champ électrique (de l'ordre de $106 \mathrm{~V} \mathrm{~m}^{-1}$ ) à $100 \mathrm{~W}$ de puissance micro-ondes incidente à une fréquence de $2,45 \mathrm{GHz}$. Un taux élevé d'inactivation d'Escherichia coli a été observé sans augmentation importante de la température $\left(8,6^{\circ} \mathrm{C}\right)$, ce qui suggère l'existence d'effets non thermiques.

Dans une étude sur les effets du rayonnement micro-ondes (à $18 \mathrm{GHz}$ ) à une température sublétale $\left(<40{ }^{\circ} \mathrm{C}\right)$ sur Escherichia coli, Shamis et al (2011) ont observé que les cellules des bactéries présentent une morphologie significativement différente de celle obtenue avec un chauffage classique. Cependant, la microscopie électronique à balayage a également montré que cet effet était temporaire, puisque la morphologie semblait revenir à son état initial après quelques minutes. Les auteurs ont proposé que l'exposition aux micro-ondes peut causer une modification électrocinétique, entraînant une plus grande porosité dans la membrane cellulaire. Des altérations de la membrane cellulaire à basse température ont également été observées par Rougier et al (2014).

Par contre, Kozempel et ses collaborateurs (2000) n'ont trouvé aucune preuve d'effets non thermiques. Les auteurs ont utilisé un dispositif micro-ondes continu avec un tube de refroidissement interne pour absorber l'énergie thermique dégagée et contrôler la température en dessous de $45^{\circ} \mathrm{C}$ dans la matrice. Le processus s'est déroulé avec un écoulement externe turbulent pour assurer une température uniforme dans le produit. Aucun effet non thermique des micro-ondes n'a été observé pour des cellules de type levure, Pediococcus sp., Escherichia coli, Listeria innocua ou Enterobacter aerogenes dans divers liquides, comme l'eau, les œufs liquides, la bière, le jus de pomme, le cidre et le jus de tomate. L'absence d'effets non thermiques a également été signalée dans le cadre d'inactivation d'Escherichia-Coli dans un gel d'agar modèle (Hamoud-Agha et al, 2013) et des spores de Clostridium sporogenes (Welt et al., 1994).

En ce qui concerne l'inactivation enzymatique, certaines études confirment l'existence d'effets non thermiques des micro-ondes, d'autres non. Parmi les études qui ont rapporté des effets athermiques, on peut citer les travaux de Latorre et al (2012). Les auteurs ont évalué l'inactivation d'enzyme de type OPP et POD chez la betterave rouge par des traitements thermiques conventionnels et micro-ondes. Étant donné que l'inactivation enzymatique était plus rapide dans les échantillons traités par microondes par rapport aux échantillons traités de façon conventionnelle soumis à des températures similaires, on pourrait en déduire l'existence d'effets non thermiques des micro-ondes.

Dans une étude rapportée par Lin et Ramaswamy (2011), le lait cru a été soumis à un chauffage isotherme conventionnel au bain-marie, à un chauffage par micro-ondes à débit continu et à un maintien thermique en continu. Dans chaque cas, les activités résiduelles de la phosphatase alcaline ont été évaluées. Les données d'inactivation ont été évaluées et ajustées à un modèle cinétique de premier ordre. Aucune inactivation enzymatique significative n'a été observée à des températures inférieures à $60^{\circ} \mathrm{C}$ avec des temps de séjour courts pendant le chauffage par micro-ondes à flux continu. Il n'y avait donc aucune preuve de l'existence d'un effet non thermique dans ces conditions. Cependant, à des températures plus élevées $\left(65-70{ }^{\circ} \mathrm{C}\right)$, l'inactivation enzymatique sous chauffage par micro-ondes à flux continu s'est produite beaucoup plus rapidement que sous chauffage conventionnel, ce qui indique l'existence d'effets spécifiques associés au chauffage par micro-ondes. Des résultats similaires ont été rapportés pour les enzymes PME dans le jus d'orange (Tajchakakavit et Ramaswamy, 1995). 
Selon Kermasha et ses collaborateurs (1993a, 1993b), des effets non thermiques dus au chauffage par micro-ondes de la lipoxygénase de soja et de la lipase dans les germe de blé ont été notifiés. Les molécules protéiques contenant les fractions polaires et chargées peuvent avoir été affectées par le champ électrique. En raison d'un champ électromagnétique fort et aléatoire, les liaisons non covalentes telles que les liaisons hydrophobes, électrostatiques et hydrogène peuvent être perturbées. Cependant, d'autres auteurs affirment que les micro-ondes ne peuvent rompre aucune liaison chimique par manque d'énergie, contrairement au rayonnement ultraviolet, visible et infrarouge qui peuvent fournir suffisamment d'énergie pour rompre les liaisons hydrogène (Ramaswamy et al., 2002).

Une autre théorie pour expliquer les effets non thermiques des micro-ondes sur les enzymes est basée sur les altérations conformationnelles des molécules. Bohr et Bohr (2000) ont étudié les changements de conformation de la protéine $\beta$-lactoglobuline pendant une exposition aux micro-ondes à $2,45 \mathrm{GHz}$ et une puissance micro-ondes incidente de $800 \mathrm{~W}$. Il a été observé que les micro-ondes pouvaient induire une dénaturation/repliage de la protéine au cours du traitement. La même observation n'a pas pu être reproduite en utilisant un chauffage conventionnel avec des profils de température similaires, ce qui indique que l'effet micro-ondes n'est pas seulement thermique. De Pomerai et ses collaborateurs (2003) ont fait état de résultats similaires. Des altérations de la conformation des protéines lors d'une exposition à des rayonnements micro-ondes sans changement de température mesurable ont été observées pour l'albumine de sérum bovin.

El Mecherfi et al (2018) ont étudié le traitement micro-ondes de protéines de lactosérum à $37^{\circ} \mathrm{C}$, $50^{\circ} \mathrm{C}, 65^{\circ} \mathrm{C}$ et $70^{\circ} \mathrm{C}$ pour réaliser la pepsinolyse et produire de grandes quantités d'hydrolysats de protéines de lactosérum bovin hydrolysées à faible propriétés allergènes. Le procédé micro-ondes a été comparé à un traitement thermique conventionnel. Les deux processus ont été analysés en profondeur en termes de cinétique thermique afin de disposer de la même histoire thermique pour les deux traitements. Les hydrolysats de pepsine obtenus par le traitement micro-ondes en continu et chauffage conventionnel ont été caractérisés par SDS-PAGE et RP-HPLC. Les résultats indiquent que les hydrolysats de protéines de lactosérum ont été obtenus seulement à $65^{\circ} \mathrm{C}$ et dans un temps plus court par rapport au traitement thermique conventionnel. Le degré d'hydrolyse obtenu sous micro-ondes est significativement plus élevé $(p<0,001)$ que celui obtenu avec chauffage conventionnel $(14,1 \%$ contre $7.1 \%$, respectivement). Les résultats démontrent ainsi que la $\beta$-lactoglobuline est soumise à une dynamique de dénaturation particulièrement intense sous l'action des micro-ondes par rapport au chauffage conventionnel.

Lopes et ses collaborateurs (2015) ont signalé que dans le cas de la peroxydase de raifort, les régions glycosylées de l'enzyme peuvent absorber efficacement le rayonnement micro-ondes. Effectivement, son dipôle peut se réorienter rapidement sous micro-ondes, détruisant ainsi la structure tridimensionnelle de l'enzyme, plus sévèrement que le chauffage conventionnel.

D'autre part, certaines études n'ont pas détecté la présence d'effets non thermiques des micro-ondes. Par exemple, Xu et ses collaborateurs (2016) ont évalué la cinétique d'inactivation de la lipase et de la lipoxygénase du germe de blé. Les échantillons ont été traités par chauffage conventionnel dans un bain d'eau chaude et par chauffage micro-ondes monomode à 2,45 GHz. En comparant les résultats des deux traitements, aucune différence significative n'a été observée et, par conséquent, aucune preuve d'effets non thermiques n'a été trouvée. Chen et al (2016) et Galvin et al (1981) ont signalé des résultats similaires pour les activités de l'acétylcholinestérase et de la créatine phosphokinase à des températures sublétales.

Siguemoto et ses collaborateurs (2018b) n'ont pas non plus signalé l'existence d'effets non thermiques lorsqu'ils ont évalué l'inactivation de la PPO, POD et de la PME dans le jus de pomme trouble par traitement micro-ondes. Dans ces travaux, de très faibles différences d'inactivation ont été obtenues entre les résultats sous chauffage conventionnel et micro-ondes. Par exemple, pour une température de $70{ }^{\circ} \mathrm{C}$, la différence moyenne entre l'activité enzymatique résiduelle prévue pour les 
deux traitements était inférieure à $2 \%$ pour la PME, $3 \%$ pour la PPO et $2 \%$ pour la POD. L'existence d'effets non thermiques a donc été écartée en raison des faibles valeurs et des incertitudes de mesure.

\section{Conclusion}

Le revue bibliographique présentée dans ce document met en évidence la complexité des traitements thermiques par micro-ondes et les paramètres influents qui permettent de prédire les interactions entre les micro-ondes et la matière. Tout d'abord, les principes de la technologie micro-ondes ont été discutés. Une présentation résumée sur la façon dont les micro-ondes interagissent avec les matrices alimentaires a été effectuée, soulignant l'importance des propriétés diélectriques du matériau au cours d'un chauffage par micro-ondes. Les principes de l'électromagnétisme et de la propagation des ondes ont également été présentés (au niveau théorique et expérimental). En particulier, la propagation au sein de guides d'ondes rectangulaires a été mise en évidence, puisque de telles structures sont couramment utilisées dans les cavités micro-ondes conventionnelles.

Puis, après avoir couvert les bases théoriques des micro-ondes et de l'électromagnétisme, le thème de la simulation numérique a été introduit. Une importance particulière a été accordée à la modélisation du chauffage micro-ondes de produits à l'état liquide car cela engendre des couplages multiphysiques importants entre les équations de l'électromagnétisme, le transfert de chaleur et la mécanique des fluides. Ainsi, les principales équations qui régissent ces phénomènes ont été mises en relation et discutées.

Dans la section suivante, l'utilisation de la technologie micro-ondes pour la transformation des aliments a été abordée, présentant les principales applications dans la recherche et l'industrie, ainsi que les avantages et les inconvénients du chauffage par micro-ondes. En particulier, l'utilisation du chauffage par micro-ondes pour l'inactivation enzymatique a été présentée. De plus, l'existence controversée d'effets non thermiques possibles sur l'inactivation enzymatique et microbienne a été discutée, rapportant certains résultats et théories des études déjà réalisées.

Ces éléments donnent donc une vue d'ensemble sur les potentielles applications de la technologie micro-ondes pour l'industrie alimentaire tout en détaillant les paramètres procédés et propriétés produits influents afin d'optimiser le traitement thermique.

\section{References}

Edgar, R.H. \& Osepchuk, J.M. (2001). Consumer, Commercial, and Industrial Microwave Ovens and Heating Systems, in: Datta, A.K., Anantheswaran, R.C. (Eds.), Handbook of Microwave Technology for Food Applications. Marcel Dekker, New York, NY, pp. 215-275.

Perino, S., Chemat, F. (2015), Chauffage micro-ondes comme éco-procédé en industrie agroalimentaire, Techniques de l’Ingénieur, F 3 070- 1 -18

İçier, F., Baysal, T. (2004a). Dielectrical Properties of Food Materials-1: Factors Affecting and Industrial Uses. Crit. Rev. Food Sci. Nutr. 44, 465-471.

Zhu, X., Guo, W., Wu, X. (2012). Frequency- and temperature-dependent dielectric properties of fruit juices associated with pasteurization by dielectric heating. J. Food Eng. 109, 258-266.

Chandrasekaran, S., Ramanathan, S., Basak, T.(2013). Microwave Food Processing - A review. Food Res. Int. 52, 243261.

Meda, V., Orsat, V., Raghavan, V. (2005). Microwave heating and the dielectric properties of foods, The Microwave Processing of Foods. Woodhead Publishing Limited.

Datta, A. K., Davidson, P.M. (2000). Special Supplement Kinetics of Microbial Inactivation for Alternative Food Processing Technologies (Microwave and Radio Frequency Processing).pdf. J. Food Sci.

Salazar-González, C., Martín-González, M.F.S., López-Malo, A., Sosa-Morales, M.E. (2012). Recent Studies Related to Microwave Processing of Fluid Foods. Food Bioprocess Technol. 5, 31-46. 
Kubo, M.T.K., Curet, S., Augusto, P.E.D., Boillereaux, L. (2018a). Artificial Neural Network for prediction of dielectric properties relevant to microwave processing of fruit juice. J. Food Process Eng. 41(6) e12815

Tang, J. (2015). Unlocking Potentials of Microwaves for Food Safety and Quality. J. Food Sci. 80, E1776-E1793.

Roebuck, B. D., Goldblith, S. a, \& Westphal, W. B. (1972). Dielectric Properties of Carbohydrate-Water Mixtures At Microwave Frequencies. Journal of Food Science, 37(2), 199-204.

Yin, C. (2012). Microwave-assisted pyrolysis of biomass for liquid biofuels production. Bioresour Technol 120, $273-284$.

Komarov, V., Wang, S., Tang, J. (2005). Permittivity and Measurements. Encycl. RF Microw. Eng. 3693-3711.

Curet, S., Rouaud, O., Boillereaux, L. (2008a). Microwave tempering and heating in a single-mode cavity: Numerical and experimental investigations. Chem. Eng. Process. Process Intensif. 47, 1656-1665.

Sosa-Morales, M.E., Valerio-Junco, L., López-Malo, a., García, H.S. (2010). Dielectric properties of foods: Reported data in the 21st Century and their potential applications. LWT - Food Sci. Technol. 43, 1169-1179.

Datta, A.K. (2001). Fundamentals of heat and moisture transport for microwaveable food product and process development, in: Datta, A.K., Anantheswaran, R.C. (Eds.), Handbook of Microwave Technology for Food Applications. Marcel Dekker, New York, NY, pp. 115-172.

Bengtsson, N. E., Risman P. O. (1971). Dielectric properties of foods at $3 \mathrm{GHz}$ as determined by a cavity perturbation technique, II. Measurements on food materials, J. Microwave Power 6(2):107-123)

Stogryn A. (1971) Equations for calculating the dielectric constant of saline water, IEEE Trans. Microwave Theory Techn. 19:733-736

İçier, F., Baysal, T. (2004b). Dielectrical Properties of Food Materials-2: Measurement Techniques. Crit. Rev. Food Sci. Nutr. 44, 473-478.

Nelson, S.O., Datta, A.K. (2001). Dielectric properties of food materials and electric field interactions, in: Datta, A.K., Anantheswaran, R.C. (Eds.), Handbook of Microwave Technology for Food Applications. Marcel Dekker, New York, NY, pp. 69-114.

Khaled, D. El, Castellano, N.N., Gázquez, J.A., Perea-Moreno, A.J., Manzano-Agugliaro, F., (2016). Dielectric spectroscopy in biomaterials: Agrophysics. Materials (Basel). 9, 1-26

Arai, M., Binner, J.G.P., Cross, T.E. (1995). Estimating errors due to sample surface roughness in microwave complex permittivity measurements obtained using a coaxial probe. Electron. Lett. 31, 115.

Chen, J., Pitchai, K., Birla, S., Negahban, M., Jones, D., Subbiah, J. (2014). Heat and mass transport during microwave heating of mashed potato in domestic oven--model development, validation, and sensitivity analysis. J. Food Sci. 79, E1991-2004.

Kraszewski, A.W. \& Nelson, S.O. (1996) Resonant Cavity PerturbationSome New Applications of an Old Measuring Technique, Journal of Microwave Power and Electromagnetic Energy, 31:3, 178-187

Dibben, D. (2001). Electromagnetics: fundamental aspects and numerical modeling, in: Datta, A.K., Anantheswaran, R.C. (Eds.), Handbook of Microwave Technology for Food Applications. Marcel Dekker, New York, NY, pp. 1-28.

Curet, S. (2008b), Traitements micro-ondes et transferts de chaleur en milieu multiphasique, thèse de l'université de Nantes

David M. Pozar (2011), Microwave Engineering, John Wiley \& Sons, 4ème edition.

Cherbanski, R. and L. Rudniak (2013). "Modelling of microwave heating of water in a monomode applicator - Influence of operating conditions." International Journal of Thermal Sciences 74: 214-229.

Sadot, M. (2018) Etude numérique et expérimentale d'un procédé de congélation assistée par micro-ondes, thèse de la Comue Université Bretagne Loire.

Regier, M., Schubert, H. (2005). Introducing microwave processing of food: principles and technologies, in: Schubert, H., Regier, M. (Eds.), The Microwave Processing of Foods. CRC Press, Boca Raton, FL, pp. 3-21.

Debionne, G. (2005), Antennes à fentes, Techniques de l'Ingénieur, E 3 300- 1 -21

Luan, D., J. Tang, P. D. Pedrow, F. Liu and Z. Tang (2016). Analysis of electric field distribution within a microwave assisted thermal sterilization (MATS) system by computer simulation. Journal of Food Engineering 188: 87-97.

S. Charmond (2009), Développement d'un four micro-ondes monomode et frittage de poudres céramiques et métallique, Institut National Polytechnique de Grenoble - INPG. 
Resurreccion Jr, F. P., D. Luan, J. Tang, F. Liu, Z. Tang, P. D. Pedrow and R. Cavalieri (2015). "Effect of changes in microwave frequency on heating patterns of foods in a microwave assisted thermal sterilization system." Journal of Food Engineering 150(0): 99-105.

Knoerzer, K., Regier, M., Schubert, H. (2011). Simulating and Measuring Transient Three - Dimensional Temperature Distributions in Microwave Processing, in: Knoerzer, K., Juliano, P., Roupas, P., Versteeg, C. (Eds.), Innovative Food Processing Technologies: Advances in Multiphysics Simulation. John Wiley \& Sons, Ltd. and Institute of Food Technologists, West Sussex, UK, pp. 131-153.

Chen, H., Tang, J. (2011). Computer Simulation for Microwave Heating 101-130.

Choi, W., Lee, S.H., Kim, C.T., Jun, S. (2015). A finite element method based flow and heat transfer model of continuous flow microwave and ohmic combination heating for particulate foods. J. Food Eng. 149, 159-170.

Liu, S., Fukuoka, M., Sakai, N. (2013). A finite element model for simulating temperature distributions in rotating food during microwave heating. J. Food Eng. 115, 49-62

Romano, V.R., Marra, F., Tammaro, U. (2005). Modelling of microwave heating of foodstuff: study on the influence of sample dimensions with a FEM approach. J. Food Eng. 71, 233-241.

Hamoud-Agha, M.M., Curet, S., Simonin, H., Boillereaux, L. (2013). Microwave inactivation of Escherichia coli K12 CIP 54.117 in a gel medium: Experimental and numerical study. J. Food Eng. 116, 315-323.

Oliveira, M.E.C., Franca, a. S. (2002). Microwave heating of foodstuffs. J. Food Eng. 53, 347-359.

Vadivambal, R., Jayas, D.S. (2010). Non-uniform Temperature Distribution During Microwave Heating of Food Materials-A Review. Food Bioprocess Technol. 3, 161-171.

Vilayannur, R.S., Puri, V.M., Anantheswaran, R.C. (1998). Size and shape effect on nonuniformity of temperature and moisture distributions in microwave heated food materials: Part II experimental validation. J. Food Process Eng. 21, $235-248$.

Liu, C.M., Wang, Q.Z., Sakai, N. (2005). Power and temperature distribution during microwave thawing, simulated by using Maxwell's equations and Lambert's law. Int. J. Food Sci. Technol. 40, 9-21.

Cheng, Y., Sakai, N., Hanzawa, T. (1997). Effects of Dielectric Properties on Temperature Distributions in Food Model during Microwave Heating. Food Sci. Technol. Int. Tokyo 3, 324-328.

Yang, H.W., Gunasekaran, S. (2004). Comparison of temperature distribution in model food cylinders based on Maxwell's equations and Lambert's law during pulsed microwave heating. J. Food Eng. 64, 445-453.

Knoerzer, K., Regier, M., Schubert, H. (2005). Simulation of microwave heating processes, in: Schubert, H., Regier, M. (Eds.), The Microwave Processing of Foods. CRC Press, Boca Raton, FL, pp. 317-333.

Chatterjee, S., Basak, T., Das, S.K. (2007). Microwave driven convection in a rotating cylindrical cavity: A numerical study. J. Food Eng. 79, 1269-1279.

Zhang, Q., Jackson, T.H., Ungan, A. (2000). Numerical modeling of microwave induced natural convection. Int. J. Heat Mass Transf. 43, 2141-2154.

Chen, Z., Li, Y., Wang, L., Liu, S., Wang, K., Sun, J., Xu, B. (2016). Evaluation of the possible non-thermal effect of microwave radiation on the inactivation of wheat germ lipase. J. Food Process Eng. 1-11.

Curet, S., Begnini, F.B., Rouaud, O., Boillereaux, L. (2015). Modeling Microwave Heating During Batch Processing of Liquid Sample in a Single Mode Cavity. 2015 COMSOL Conf.

Klinbun, W., Rattanadecho, P. (2016). Investigation into heat transfer and fluid flow characteristics of liquid two-layer and emulsion in microwave processing. Int. Commun. Heat Mass Transf. 70, 115-126.

Ratanadecho, P., Aoki, K., Akahori, M. (2002). A numerical and experimental investigation of the modeling of microwave heating for liquid layers using a rectangular wave guide (effects of natural convection and dielectric properties). Appl. Math. Model. 26, 449-472.

Salvi, D., Boldor, D., Aita, G.M., Sabliov, C.M. (2011). COMSOL Multiphysics model for continuous flow microwave heating of liquids. J. Food Eng. 104, 422-429.

Tuta, S., Palazoglu, T.K. (2017). Finite element modeling of continuous-flow microwave heating of fluid foods and experimental validation. J. Food Eng. 192, 79-92.

Yeong, S.P., Law, M.C., Vincent Lee, C.C., Chan, Y.S. (2017). Modelling batch microwave heating of water. IOP Conf. Ser. Mater. Sci. Eng. 217. 
Zhu, J., Kuznetsov, a. V., Sandeep, K.P. (2007). Mathematical modeling of continuous flow microwave heating of liquids (effects of dielectric properties and design parameters). Int. J. Therm. Sci. 46, 328-341.

Norton, T., Sun, D.-W. (2006). Computational fluid dynamics (CFD) - an effective and efficient design and analysis tool for the food industry: A review. Trends Food Sci. Technol. 17, 600-620.

Scott, G., Richardson, P. (1997). The application of computational fluid dynamics in the food industry. Trends Food Sci. Technol. 8, 119-124.

Ballard, T.S., Mallikarjunan, P., Zhou, K., O'Keefe, S. (2010). Microwave-assisted extraction of phenolic antioxidant compounds from peanut skins. Food Chem. 120, 1185-1192.

Bozkir, H., Baysal, T. (2017a). Concentration of apple juice using a vacuum microwave evaporator as a novel technique: Determination of quality characteristics. J. Food Process Eng. 40, e12535.

Cañumir, J. a, Celis, J.E., de Bruijn, J., Vidal, L. V (2002). Pasteurisation of Apple Juice by Using Microwaves. Leb. undTechnologie 35, 389-392.

Chahbani, A., Fakhfakh, N., Balti, M.A., Mabrouk, M., Elhatmi, H., Zouari, N., Kechaou, N., (2018). Microwave drying effects on drying kinetics, bioactive compounds and antioxidant activity of green peas (Pisum sativum L.). Food Biosci. 25, 32-38.

Dahmoune, F., Nayak, B., Moussi, K., Remini, H., Madani, K. (2015). Optimization of microwave-assisted extraction of polyphenols from Myrtus communis L. leaves. Food Chem. 166, 585-595.

Güneş, B., Bayindirli, A. (1993). Peroxidase and lipoxygenase inactivation during blanching of green beans, green peas and carrots. LWT - Food Sci. Technol.

Kim, W.J., Park, S.H., Kang, D.H. (2018). Inactivation of foodborne pathogens influenced by dielectric properties, relevant to sugar contents, in chili sauce by $915 \mathrm{MHz}$ microwaves. Lwt 96, 111-118.

Luter, L., Wyslouzil, W., Kashyap, S.C. (1982). The Destruction of Aflatoxins in Peanuts by Microwave Roasting. Can. Inst. Food Sci. Technol. J. 15, 236-238.

Marić, M., Grassino, A.N., Zhu, Z., Barba, F.J., Brnčić, M., Rimac Brnčić, S. (2018). An overview of the traditional and innovative approaches for pectin extraction from plant food wastes and by-products: Ultrasound-, microwaves-, and enzyme-assisted extraction. Trends Food Sci. Technol. 76, 28-37.

Monteiro, R.L., Link, J. V., Tribuzi, G., Carciofi, B.A.M., Laurindo, J.B. (2018). Microwave vacuum drying and multiflash drying of pumpkin slices. J. Food Eng. 232, 1-10.

Pérez-Grijalva, B., Herrera-Sotero, M., Mora-Escobedo, R., Zebadúa-García, J.C., Silva-Hernández, E., Oliart-Ros, R., Pérez-Cruz, C., Guzmán-Gerónimo, R (2018). Effect of microwaves and ultrasound on bioactive compounds and microbiological quality of blackberry juice. LWT - Food Sci. Technol. 87, 47-53.

Siguemoto, É.S., Gut, J.A.W., Martinez, A., Rodrigo, D. (2017). Inactivation kinetics of Escherichia coli O157:H7 and Listeria monocytogenes in apple juice by microwave and conventional thermal processing. Innov. Food Sci. Emerg. Technol.

Silva, F.A., Marsaioli, A., Maximo, G.J., Silva, M.A.A.P., Gonçalves, L.A.G. (2006). Microwave assisted drying of macadamia nuts. J. Food Eng. 77, 550-558.

Simić, V.M., Rajković, K.M., Stojičević, S.S., Veličković, D.T., Nikolić, N.Č., Lazić, M.L., Karabegović, I.T. (2016). Optimization of microwave-assisted extraction of total polyphenolic compounds from chokeberries by response surface methodology and artificial neural network. Sep. Purif. Technol. 160, 89-97.

Taher, B.J., Farid, M.M. (2001). Cyclic microwave thawing of frozen meat: Experimental and theoretical investigation. Chem. Eng. Process. 40, 379-389.

Xanthakis, E., Le-Bail, a., Ramaswamy, H. (2014). Development of an innovative microwave assisted food freezing process. Innov. Food Sci. Emerg. Technol. 26, 176-181.

Antunes, E., Jacob, M. V., Brodie, G., Schneider, P.A. (2018). Microwave pyrolysis of sewage biosolids: Dielectric properties, microwave susceptor role and its impact on biochar properties. J. Anal. Appl. Pyrolysis 129, 93-100.

Chee Loong, T., Idris, A. (2014). Rapid alkali catalyzed transesterification of microalgae lipids to biodiesel using simultaneous cooling and microwave heating and its optimization. Bioresour. Technol. 174, 311-315.

Schiffmann, R.F. (2001). Microwave processes for the food industry, in: Datta, A.K., Anantheswaran, R.C. (Eds.), Handbook of Microwave Technology for Food Applications. Marcel Dekker, New York, NY, pp. 299-353. 
Guo, Q., Sun, D.W., Cheng, J.H., Han, Z. (2017). Microwave processing techniques and their recent applications in the food industry. Trends Food Sci. Technol. 67, 236-247.

Marszałek, K., Mitek, M., Skąpska, S. (2015). Effect of Continuous Flow Microwave and Conventional Heating on the Bioactive Compounds, Colour, Enzymes Activity, Microbial and Sensory Quality of Strawberry Purée. Food Bioprocess Technol. 8, 1864-1876.

Cano, M.P., Hernandez, a., Ancos, B. (1997). High pressure and temperature effects on enzyme inactivation in strawberry and orange products. J. Food Sci. 62, 85-88.

Monteiro, R.L., Link, J. V., Tribuzi, G., Carciofi, B.A.M., Laurindo, J.B. (2018). Microwave vacuum drying and multiflash drying of pumpkin slices. J. Food Eng. 232, 1-10.

Salvi, D., Ortego, J., Arauz, C., Sabliov, C.M., Boldor, D. (2009). Experimental study of the effect of dielectric and physical properties on temperature distribution in fluids during continuous flow microwave heating. J. Food Eng. 93, 149-157.

Anantheswaran, R.C., Liu, L. (1994). Effect of viscosity and salt concentration on microwave heating of model nonNewtonian liquid foods in a cylindrical container. Int. Microw. Power Inst.

Prosetya, H., Datta, A. (1991). Batch microwave heating of liquids. An experimental study. J. Microw. Power Electromagn. Energy 26, 215-226.

Cinquanta, L., Albanese, D., Cuccurullo, G., Di Matteo, M. (2010). Effect on orange juice of batch pasteurization in an improved pilot-scale microwave oven. J. Food Sci. 75, 46-50.

Cuccurullo, G., Giordano, L., Metallo, A., Cinquanta, L. (2017). Influence of mode stirrer and air renewal on controlled microwave drying of sliced zucchini. Biosyst. Eng. 158, 95-101.

Kurniawan, H., Alapati, S., Che, W.S.,(2015). Effect of mode stirrers in a multimode microwave-heating applicator with the conveyor belt. Int. J. Precis. Eng. Manuf. - Green Technol. 2, 31-36.

Ye, J., Hong, T., Wu, Y., Wu, L., Liao, Y., Zhu, H., Yang, Y., Huang, K. (2017). Model stirrer based on a multi-material turntable for microwave processing materials. Materials (Basel). 10, 1-13

Gunasekaran, S., Yang, H.W. (2007). Effect of experimental parameters on temperature distribution during continuous and pulsed microwave heating. J. Food Eng. 78, 1452-1456.

Ren, G., Chen, F. (1998). Drying of American ginseng (Panax quinquefolium) roots by microwave-hot air combination. J. Food Eng. 35, 433-443.

Atuonwu, J.C., Tassou, S.A. (2018). Quality assurance in microwave food processing and the enabling potentials of solidstate power generators: A review. J. Food Eng. 234, 1-15.

Anantheswaran, R.C., Ramaswamy, H.S. (2001). Bacterial destruction and enzyme inactivation during microwave heating, in: Datta, A.K., Anantheswaran, R.C. (Eds.), Handbook of Microwave Technology for Food Applications. Marcel Dekker, New York, NY, pp. 191-215.

Ramaswamy, H.S., Koutchma, T., Tajchakavit, S. (2002). Enhanced thermal effects under microwave heating conditions, in: Welti-Chanes, J., Barbosa-Cánovas, G.V., Aguilera, J.M. (Eds.), Engineering and Food for the 21st Century. CRC Press, Boca Raton, FL.

Shamis, Y., Croft, R., Taube, A., Crawford, R.J., Ivanova, E.P. (2012). Review of the specific effects of microwave radiation on bacterial cells. Appl. Microbiol. Biotechnol. 96, 319-325.

Huang, K., Yang, X., Hua, W., Jia, G., Yang, L. (2009). Experimental evidence of a microwave non-thermal effect in electrolyte aqueous solutions. New J. Chem. 33, 1486-1489.

Shazman, A., Mizrahi, S., Cogan, U., Shimoni, E. (2007). Examining for possible non-thermal effects during heating in a microwave oven. Food Chem. 103, 444-453.

Dreyfuss, M.S., Chipley, J.R. (1980). Comparison of effects of sublethal microwave radiation and conventional heating on the metabolic activity of Staphylococcus aureus. Appl. Environ. Microbiol. 39, 13-16.

Zhang, Y.L., Zeng, B.Q., Zhang, H. (2012). A $2.45 \mathrm{GHz}$ reentarnt coaxial cavity for liquid sterilization based on nonthermal microwave effect. Prog. Electromagn. Res. C 33, 145-156.

Shamis, Y., Taube, A., Mitik-Dineva, N., Croft, R., Crawford, R.J., Ivanova, E.P. (2011). Specific electromagnetic effects of microwave radiation on Escherichia coli. Appl. Environ. Microbiol. 77, 3017-3022. 
Rougier, C., Prorot, A., Chazal, P., Leveque, P., Leprat, P. (2014). Thermal and nonthermal effects of discontinuous microwave exposure (2.45 Gigahertz) on the cell membrane of Escherichia coli. Appl. Environ. Microbiol. 80, 4832 4841.

Kozempel, M., Cook, R.D., Scullen, O.J., Annous, B.A. (2000). Nonthermal Effects of Microwave Energy on Microorganisms At Low Temperature '. J. Food Process. Preserv. 287-301.

Welt, B.A., Tong, C.H., Rossen, J.L., Lund, D.B. (1994). Effect of microwave radiation on inactivation of Clostridium sporogenes (PA 3679) spores. Appl. Environ. Microbiol. 60, 482-488.

Latorre, M.E., Bonelli, P.R., Rojas, A.M., Gerschenson, L.N. (2012). Microwave inactivation of red beet (Beta vulgaris L. var. conditiva) peroxidase and polyphenoloxidase and the effect of radiation on vegetable tissue quality. J. Food Eng. 109, 676-684.

Lin, M., Ramaswamy, H.S. (2011). Evaluation of phosphatase inactivation kinetics in milk under continuous flow microwave and conventional heating conditions. Int. J. Food Prop. 14, 110-123.

Tajchakavit, S., Ramaswamy, H.S. (1995). Continuous-flow microwave heating of orange juice: evidence of nonthermal effects. J. Microwave Power Electromagn. Energy.

Kermasha, S., Bisakowski, B., Ramaswamy, H., van de Voort, F. (1993a). Comparison of microwave, conventional and combination heat treatments on wheat germ lipase activity. Int. J. Food Sci. Technol. 28, 617-623.

Kermasha, S., Bisakowski, B., Ramaswamy, H., Van de Voort, F.R. (1993b). Thermal and microwave inactivation of soybean lipoxygenase. LWT - Food Sci. Technol.

Bohr, H., Bohr, J. (2000). Microwave-enhanced folding and denaturation of globular proteins. Phys. Rev. E - Stat. Physics, Plasmas, Fluids, Relat. Interdiscip. Top. 61, 4310-4314.

K. El Mecherfi, S. Curet, R. Lupi, C. Larré, O. Rouaud, Y. Choiset, H. Rabesona, T. Haertlé (2018). Combined microwave processing and enzymatic proteolysis of bovine whey proteins: the impact on bovine $\beta$-lactoglobulin allergenicity, Journal of Food Science and Technology. https://doi.org/10.1007/s13197-018-3471-9

Lopes, L.C., Barreto, M.T.M., Gonçalves, K.M., Alvarez, H.M., Heredia, M.F., de Souza, R.O.M. a, Cordeiro, Y., Dariva, C., Fricks, A.T. (2015). Stability and structural changes of horseradish peroxidase: microwave versus conventional heating treatment. Enzyme Microb. Technol. 69, 10-8.

Xu, B., Wang, L.K., Miao, W.J., Wu, Q.F., Liu, Y.X., Sun, Y., Gao, C. (2016). Thermal versus microwave inactivation kinetics of lipase and lipoxygenase from wheat germ. J. Food Process Eng. 39, $247-255$.

Chen, Z., Li, Y., Wang, L., Liu, S., Wang, K., Sun, J., Xu, B. (2016). Evaluation of the possible non-thermal effect of microwave radiation on the inactivation of wheat germ lipase. J. Food Process Eng. 1-11.

Galvin, M.J., Parks, D.L., McRee, D.I. (1981). Influence of $2.45 \mathrm{GHz}$ microwave radiation on enzyme activity. Radiat. Environ. Biophys. 19, 149-156.

Siguemoto, É.S., Pereira, L.J., Gut, J.A.W. (2018b). Inactivation Kinetics of Pectin Methylesterase, Polyphenol Oxidase, and Peroxidase in Cloudy Apple Juice under Microwave and Conventional Heating to Evaluate Non-Thermal Microwave Effects. Food Bioprocess Technol. 1-11. 